\title{
Thermal Stability of Pre-annealed Hydrogen-loaded Fiber Bragg Gratings As A Function of the Fiber-Phase Mask Distance
}

\section{By}

\author{
Lingyun Xiong \\ A thesis submitted to \\ The Faculty of Graduate Studies and Research \\ in partial fulfilment of the requirements for the degree of \\ Master of Applied Science
}

Ottawa-Carleton Institute for Electrical and Computer Engineering

Department of Electronics

Carleton University

Ottawa, Ontario, Canada

September 2007

Copyright $\mathbb{C}$ Lingyun Xiong, 2007 


$\begin{array}{ll}\begin{array}{l}\text { Library and } \\ \text { Archives Canada }\end{array} & \begin{array}{l}\text { Bibliothèque et } \\ \text { Archives Canada }\end{array} \\ \begin{array}{l}\text { Published Heritage } \\ \text { Branch }\end{array} & \begin{array}{l}\text { Direction du } \\ \text { Patrimoine de l'édition }\end{array} \\ \begin{array}{l}\text { 395 Wellington Street } \\ \text { Ottawa ON K1A ON4 }\end{array} & \begin{array}{l}\text { 395, rue Wellington } \\ \text { Ottawa ON K1A ON4 } \\ \text { Canada }\end{array}\end{array}$

Your file Votre référence ISBN: 978-0-494-33675-5 Ourfile Notre référence ISBN: 978-0-494-33675-5

NOTICE:

The author has granted a nonexclusive license allowing Library and Archives Canada to reproduce, publish, archive, preserve, conserve, communicate to the public by telecommunication or on the Internet, loan, distribute and sell theses worldwide, for commercial or noncommercial purposes, in microform, paper, electronic and/or any other formats.

The author retains copyright ownership and moral rights in this thesis. Neither the thesis nor substantial extracts from it may be printed or otherwise reproduced without the author's permission.
AVIS:

L'auteur a accordé une licence non exclusive permettant à la Bibliothèque et Archives Canada de reproduire, publier, archiver, sauvegarder, conserver, transmettre au public par télécommunication ou par l'Internet, prêter, distribuer et vendre des thèses partout dans le monde, à des fins commerciales ou autres, sur support microforme, papier, électronique et/ou autres formats.

L'auteur conserve la propriété du droit d'auteur et des droits moraux qui protège cette thèse. $\mathrm{Ni}$ la thèse ni des extraits substantiels de celle-ci ne doivent être imprimés ou autrement reproduits sans son autorisation.
In compliance with the Canadian

Privacy Act some supporting forms may have been removed from this thesis.

While these forms may be included in the document page count, their removal does not represent any loss of content from the thesis.
Conformément à la loi canadienne sur la protection de la vie privée, quelques formulaires secondaires ont été enlevés de cette thèse.

Bien que ces formulaires aient inclus dans la pagination, il n'y aura aucun contenu manquant. 


\begin{abstract}
The objective of this study is to investigate the issue of the thermal stability of hydrogenloaded fiber Bragg gratings. Gratings with various refractive index contrasts have been fabricated in hydrogen-loaded single mode fibers by changing the fiber/phase mask distance. In order to replicate standard industrial practice, all of the hydrogen-loaded gratings are subjected a pre-annealing process to remove the most unstable part of the index change (30\% of the index modulation). The accelerated aging tests at different temperatures carried out on these pre-annealed gratings has demonstrated that a larger DC refractive index change formed at the larger fiber/mask distance can improve the thermal stability of the grating reflectivity. However, the improved stability is only observed for relatively large changes of the fiber/phase mask distance (several hundred microns). This rules out fiber/phase mask distance inaccuracies as the main cause of the observed differences in the thermal stability of mass-produced gratings in industry. It was also found that none of those existing standard accelerated aging models could accurately describe the thermal decay behavior of our pre-annealed hydrogen-loaded fiber Bragg gratings. Additional measurements are presented in the thesis on grating growth curves of saturated hydrogen-loaded gratings that show an anomalous behavior never reported before but consistent with the hypothesis mentioned above. All of these findings support the previously reported hypothesis that the DC and AC components of the UV-induced index change of hydrogen-loaded gratings involve different photosensitivity mechanisms.
\end{abstract}




\section{Acknowledgements}

I would like to express my sincere gratitude to my supervisor, Professor Jacques Albert. He provided the initial inspiration for this work and gave critical guidance throughout the process. His patience and support made me to keep my courage to overcome the difficulties and accomplish this work.

I would also like to thank my colleagues and friends Chengkun Chen, Amir Jafari, Alexander Anderyuk, Albane Laronche, and Kuanpei Yap for their help and advice, as well as Prof. Matthieu Lancry from the University of Paris-Sud who read this thesis and provided useful comments. Thanks should go to Ksenia Yadav for her great help on proofreading this thesis.

The final version of this thesis also benefited greatly from the advice provided by the members of the thesis defense jury, especially Professors Jianping Yao and Alan Steele. Finally, I would like to thank my parents and sister for their endless love and support all through my life. 


\section{Table of Contents}

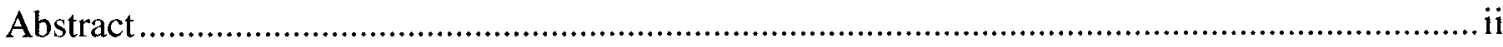

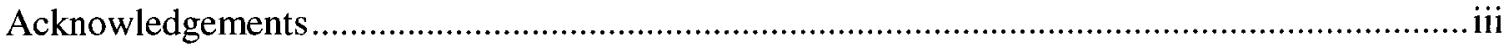

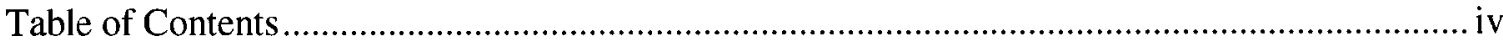

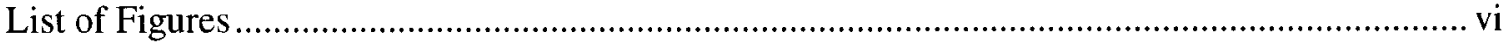

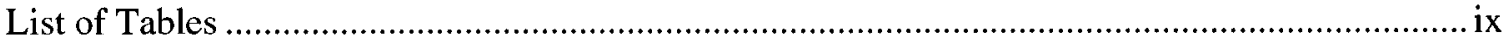

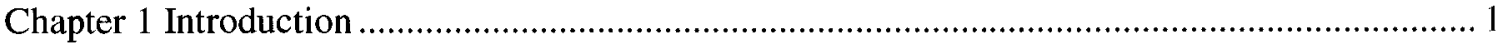

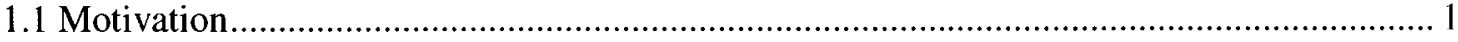

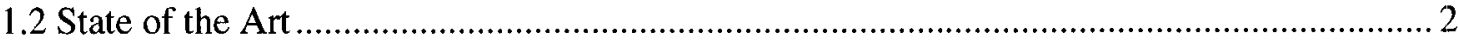

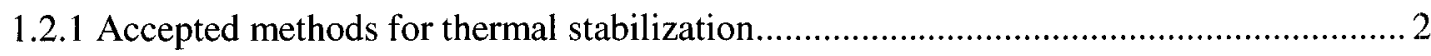

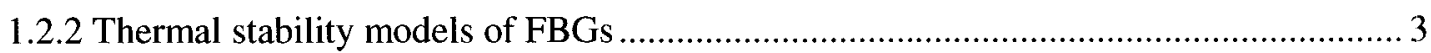

1.2.3 Thermal stabilization by Pre- and Post-exposure techniques .................................... 3

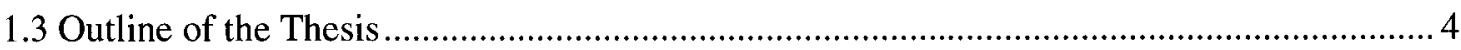

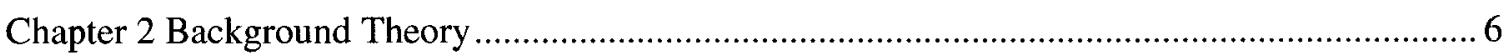

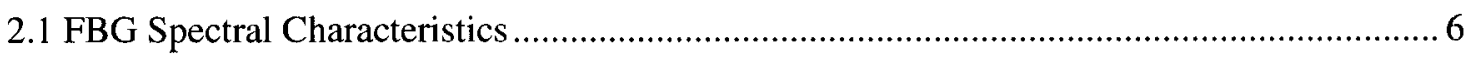

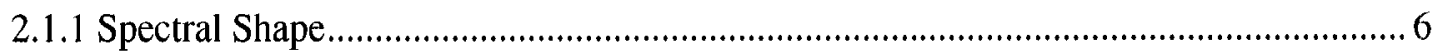

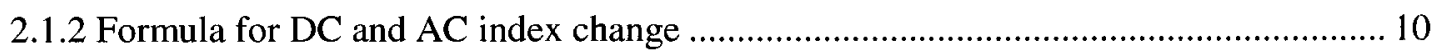

2.1.3 Influence of fiber/phase mask distance (writing distance) on contrast ........................ 13

2.2 Thermal Stability Models of Fiber Bragg Gratings ..................................................... 15

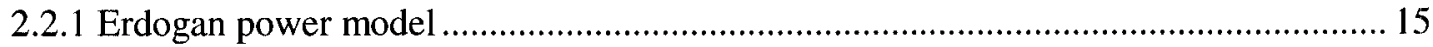

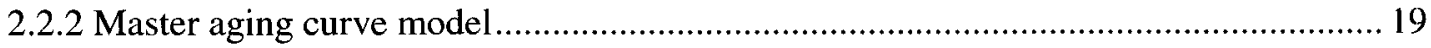

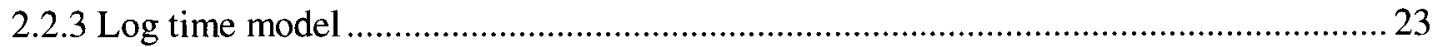

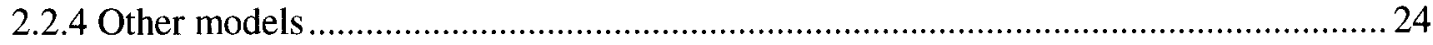

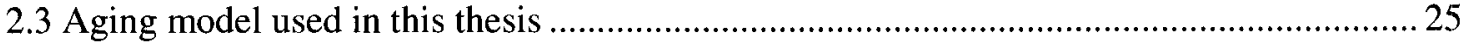

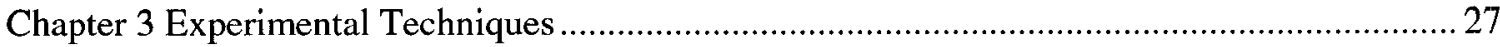

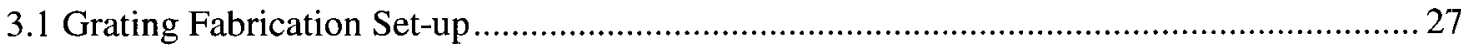

3.1.1 Fiber description and Hydrogen loading technique ............................................. 27

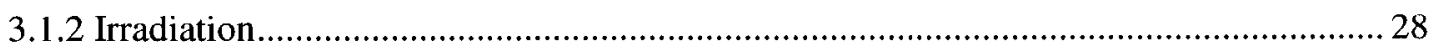

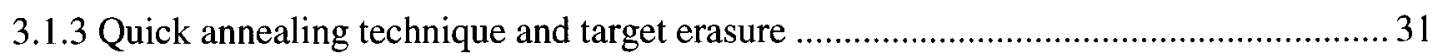

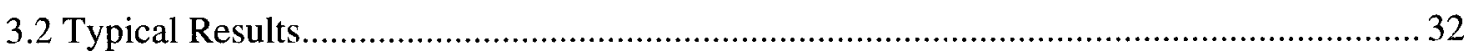

3.2.1 Uniform fiber Bragg grating spectra (Transmission and Reflection) ......................... 32

iv 
3.2.2 Grating growth 34

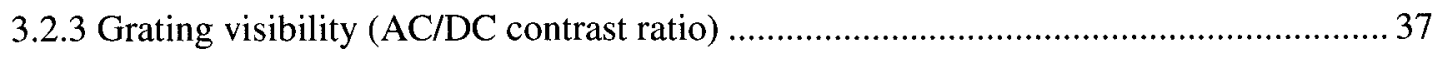

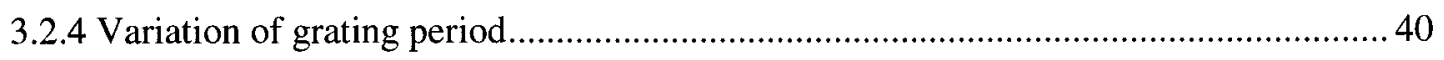

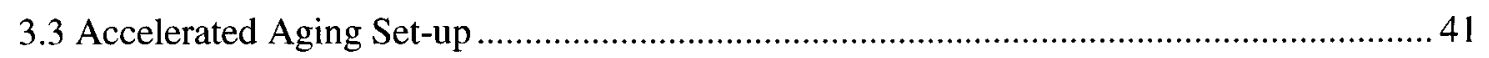

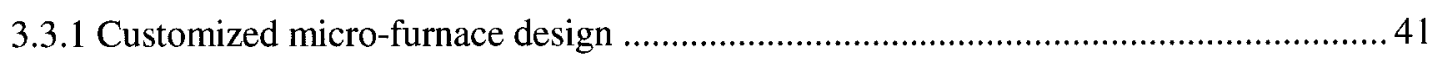

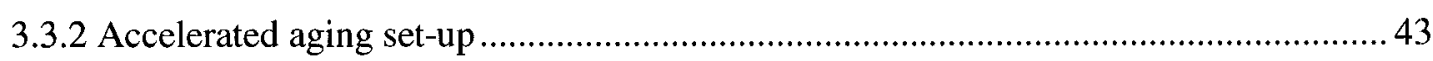

3.3.3 Problems and solutions for data processing method ................................................ 45

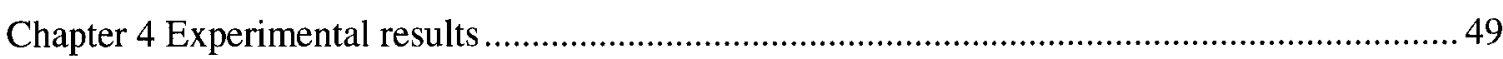

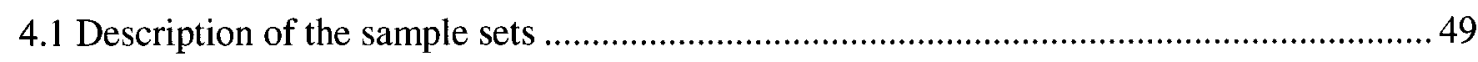

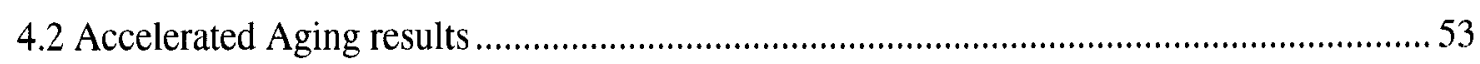

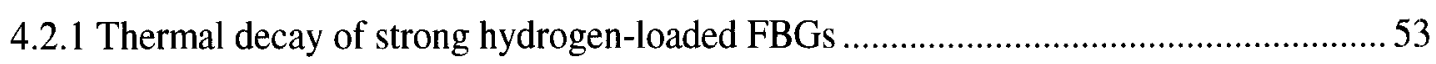

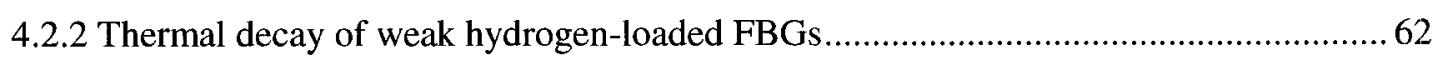

4.2.3 Bragg wavelength thermal evolution during accelerated aging .................................6 68

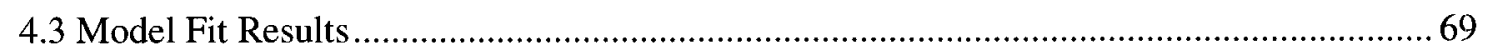

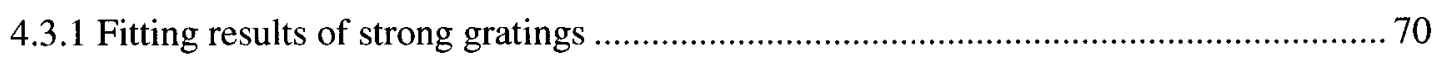

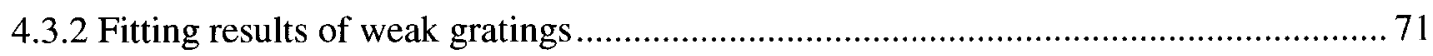

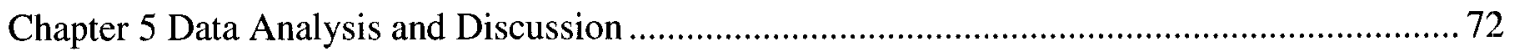

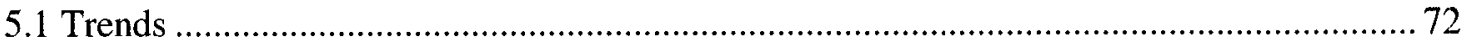

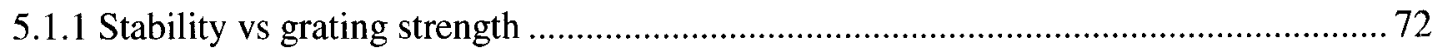

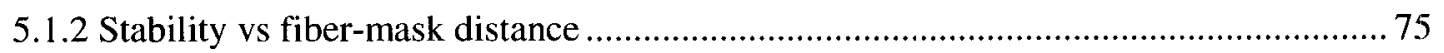

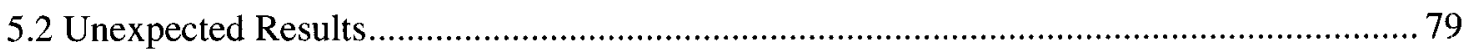

5.2.1 Deviation of annealing data for two nominally identical gratings ............................. 79

5.2.2 Large deviation in a single thermal annealing data................................................. 83

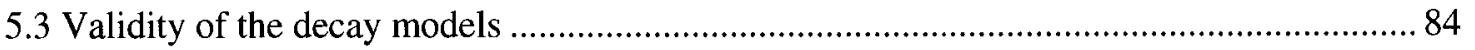

5.3.1 Validity of the Erdogan power law model, the log time model, and the simple power

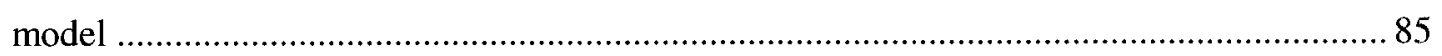

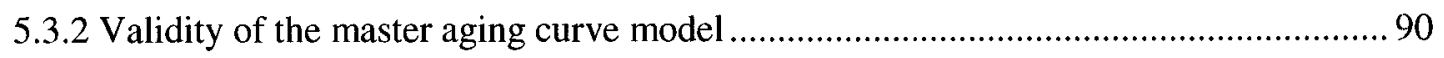

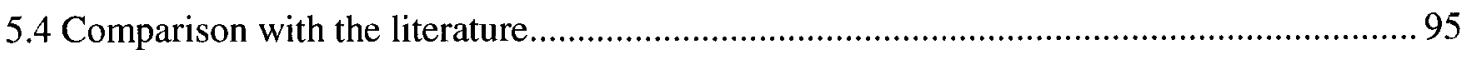

Chapter 6 Conclusions and Recommendations for Future Work............................................ 102

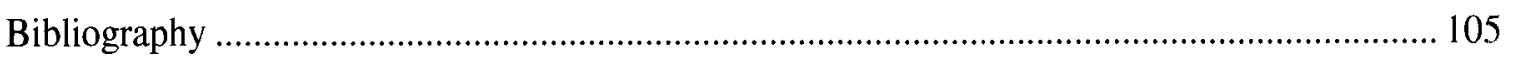




\section{List of Figures}

Fig 2.1 Schematic of a uniform Bragg grating with constant index of modulation amplitude and period [2]

Fig 2.2 A typical simulated reflection spectrum of a uniform fiber Bragg grating....................... 10

Fig 2.3 Schematic of the phase mask technique for inscribing fiber Bragg gratings

Fig 2.4 Thermal decay of normalized ICC for two gratings as a function of decay time at temperature of $350^{\circ} \mathrm{C}$ and $550^{\circ} \mathrm{C}[15]$

Fig 2.5 (a) The power law factor $A$ varying as a function of temperature (b) The power law exponent factor $\alpha$ varying as a function of temperature [15]

Fig 2.6 Diagram of the physical model for (a) electrons excited by UV irradiation during grating formation, and (b) thermal depopulation of traps during thermal decay of a grating [15] ...........21

Fig 2.7 Plot of the NICC as a function of the demarcation energy $E_{d}[15]$................................21

Fig 2.8 Plot of the NICC of a hydrogen loaded fiber grating as a function of time [17] .............24

Fig 3.1 Schematic of a fiber Bragg grating fabrication system (Top view) ................................ 31

Fig 3.2 Transmission and reflection spectrum of a uniform fiber Bragg grating........................... 33

Fig 3.3 Grating growth of both the mean refractive index change and the refractive index modulation of gratings written at fiber/mask distance of $100 \mu \mathrm{m}$ and $600 \mu \mathrm{m}$............................35

Fig 3.4 The dependence of grating index contrast (AC/DC) on the fiber/phase mask distance for

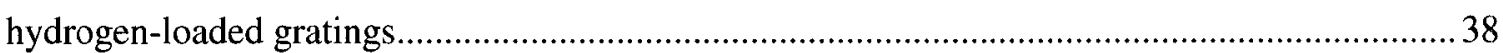

Fig 3.5 Grating growths of refractive index change for 6 gratings written at $600 \mu \mathrm{m}$.................40

Fig 3.6 The design diagram of a rectangular tube employed as micro furnace. ........................... 42

Fig 3.7 Schematic of the accelerated aging experimental setup for the measurement of multiple gratings.

Fig 3.8 Normalized change in the grating index modulation as a function of temperature $\mathrm{T}\left({ }^{\circ} \mathrm{C}\right) 47$ Fig 4.1 Isothermal decay of normalized integrated coupling constant (i.e. normalized refractive index modulation amplitude, normalized to the initial value at annealing temperature) for strong gratings as a function of annealing time at temperature of $300^{\circ} \mathrm{C}$ (a) linear time scale; (b) logarithmic time scale.

Fig 4.2 Isothermal decay of normalized integrated coupling constant (i.e. normalized refractive index modulation amplitude, normalized to the initial as-written value) for strong gratings as a 
function of annealing time at temperature of $300^{\circ} \mathrm{C}$ (a) linear time scale; (b) logarithmic time scale.

Fig 4.3 Isothermal decay of NICC (normalized to the initial value at annealing temperature) for strong gratings as a function of annealing time at temperature of $250^{\circ} \mathrm{C}$ (a) linear time scale; (b) logarithmic time scale.

Fig 4.4 Isothermal decay of NICC (normalized to the initial as-written value) for strong gratings as a function of annealing time at temperature of $250^{\circ} \mathrm{C}$ (a) linear time scale; (b) logarithmic time scale.

Fig 4.5 Isothermal decay of NICC (normalized to the initial value at annealing temperature) for strong gratings as a function of annealing time at temperature of $200^{\circ} \mathrm{C}$ (a) linear time scale; (b) logarithmic time scale.

Fig 4.6 Isothermal decay of NICC (normalized to the initial as-written value) for strong gratings as a function of annealing time at temperature of $200^{\circ} \mathrm{C}$ (a) linear time scale; (b) logarithmic time scale.

Fig 4.7 Isothermal decay of NICC (normalized to the initial value at annealing temperature) for weak gratings as a function of annealing time at temperature of $300^{\circ} \mathrm{C}$ (a) linear time scale; (b) logarithmic time scale.

Fig 4.8 Isothermal decay of NICC (normalized to the initial as-written value) for weak gratings as a function of annealing time at temperature of $300^{\circ} \mathrm{C}$ (a) linear time scale; (b) logarithmic time scale.

Fig 4.9 Isothermal decay of NICC (normalized to the initial value at annealing temperature) for weak gratings as a function of annealing time at temperature of $250^{\circ} \mathrm{C}$ (a) linear time scale; (b) logarithmic time scale.

Fig 4.10 Isothermal decay of NICC (normalized to the initial as-written value) for weak gratings as a function of annealing time at temperature of $250^{\circ} \mathrm{C}$ (a) linear time scale; (b) logarithmic time scale.

Fig 4.11 Isothermal decay of NICC (normalized to the initial value at annealing temperature) as a function of annealing time at temperature of $200^{\circ} \mathrm{C}$ (a) linear time scale; (b) logarithmic time scale.

Fig 4.12 Isothermal decay of NICC (normalized to the initial as-written value) for weak gratings as a function of annealing time at temperature of $200^{\circ} \mathrm{C}$ (a) linear time scale; (b) logarithmic time scale. 
Fig 5.1 Thermal decays of strong and weak hydrogen-loaded pre-annealed gratings with writing distance of $100 \mu \mathrm{m}$ (a) NICC normalized to as-written value; (b) NICC normalized to initial value prior to annealing .73

Fig 5.2 (replica) Thermal decays of hydrogen-loaded pre-annealed gratings written at various distances in terms of NICC normalized to as-written value (a) strong gratings at $300^{\circ} \mathrm{C}$; (b) strong gratings at $250^{\circ} \mathrm{C}$; (c) strong gratings at $200^{\circ} \mathrm{C}$; (d) weak gratings at $250{ }^{\circ} \mathrm{C}$. .76 Fig 5.3 Thermal decay curves for four similar hydrogen-loaded gratings written at $100 \mu \mathrm{m}$ during annealing at $300{ }^{\circ} \mathrm{C}$. .80

Fig 5.4 (replica) Thermal decays of hydrogen-loaded pre-annealed gratings written at various distances at $200{ }^{\circ} \mathrm{C}$ (a) in terms of NICC normalized to as-written value; (b) in terms of NICC normalized to initial value prior to annealing.

Fig 5.5 (replica) Thermal decays of NICC (normalized to as-written value) for weak gratings at $300^{\circ} \mathrm{C}$

Fig 5.6 Thermal decay of the NICC for strong gratings fitted with the simple power model, the log time model, and the Erdogan power model. .85

Fig 5.7 The temperature dependence of Erdogan power-law exponent factor $\alpha$ (Inset shows the temperature dependence of $\alpha$ from ref [32] for comparison). .88

Fig 5.8 The temperature dependence of the fitting coefficients (a) $a$ and (b) $b$ for simple power model; (c) $\tau$ and (d) $k$ for log time model. 90

Fig 5.9 Master aging curves obtained from thermal annealing at $200^{\circ} \mathrm{C}, 250^{\circ} \mathrm{C}$ and $300^{\circ} \mathrm{C}$ for pre-annealed gratings written at (a) $100 \mu \mathrm{m}$; (b) $350 \mu \mathrm{m}$.

Fig 5.9 Master aging curves obtained from thermal annealing at $200{ }^{\circ} \mathrm{C}, 250^{\circ} \mathrm{C}$ and $300{ }^{\circ} \mathrm{C}$ for pre-annealed gratings written at (c) $600 \mu \mathrm{m}$.

Fig 5.10 Isothermal decay of normalized index modulation as a function of annealing time for both post-exposure gratings and untreated gratings at different temperatures [22].

Fig 5.11 The growth of both the mean refractive index change and refractive index modulation during the formation of saturated gratings written at both 600 and $1000 \mu \mathrm{m}$. 


\section{List of Tables}

Table 3.1 Grating index contrast versus fiber/phase mask distance for gratings. ...........................38

Table 4.1 Characteristics of index change for strong gratings ................................................51

Table 4.2 Characteristics of index change for weak gratings ...............................................52

Table 4.3 Fitting results for the NICC isothermal decay (normalized to the initial value at

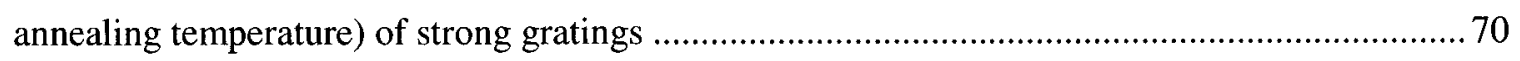

Table 4.4 Fitting results for the NICC isothermal decay (normalized to the as-written value) of

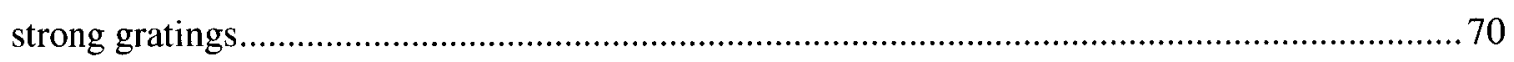

Table 4.5 Fitting results for the NICC isothermal decay (normalized to the initial value at

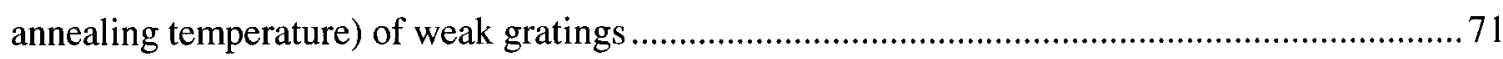

Table 4.6 Fitting results for the NICC isothermal decay (normalized to the as-written value) of weak gratings

Table 5.1 Fitting results of thermal decay of pre-annealed gratings with master aging curve model.

Table 5.2 The decrease in the Bragg wavelength induced by the accelerated aging experiment on pre-annealed gratings 98 


\section{Chapter 1}

\section{Introduction}

\subsection{Motivation}

Ever since their discovery by Hill et al in 1978 [1], fiber Bragg gratings (FBGs) received much interest and have been developed to a mature technology as an optical component. With a spatially periodic UV-induced refractive index modulation along the fiber axis, FBGs can selectively reflect light at a certain wavelength which satisfies the Bragg condition. The FBG has several advantages such as compact in-fiber geometry, cost effectiveness, low insertion loss, and flexibility of achieving desired spectral profiles. Therefore, FBGs have been playing a key role in the optic communication and fiber optic sensing system [2-3].

FBGs have been utilized in a wide range of applications: dense wavelength division multiplexing (DWDM), optical fiber lasers, dispersion compensation, and fiber optic sensing. The spectral characteristics of a FBG are suitable for a DWDM filter because it is able to satisfy the requirement of a sharp and flat-top reflection spectrum [4-6]. FBGs have also found an application as narrow band wavelength selective filters in different types of optical fiber lasers [7-9]. The chromatic dispersion experienced by optical signals during the propagation along a long distance fiber can be compensated by a chirped fiber Bragg grating [10-11]. One of the most promising applications of FBGs other than optical communication is in optical sensing. Since the center wavelength of the reflection spectrum of a FBG can be easily changed as a function of some external 
parameters, FBGs have been demonstrated as excellent wavelength encoded sensors for measuring temperature, strain, and pressure [12-14].

However, most applications require extremely high tolerances for the optical properties of the gratings over a long time, and the thermal stability of gratings is of prime importance for this concern. Investigation of the thermal decay of the UV-induced refractive index change in fiber gratings as a function of time and temperature is necessary to assess their validity for long-term applications.

\subsection{State of the Art}

\subsubsection{Accepted methods for thermal stabilization}

Thermal decay experiments have demonstrated that the UV-induced index change in the grating is not a permanent change, and the grating strength will decay over a long time even at room temperature. One approach used to stabilize the gratings, known as accelerated aging [2], is to pre-anneal the grating at a temperature that exceeds the anticipated serviceable temperature of the grating-based device. After this process, if operated at lower temperature, the grating generally will not experience further thermal decay. This should attribute to the characteristics of the grating's thermal decay curve, which starts as a fast decay followed by a substantially slower decay as the time progresses. The aim of accelerated aging is to help gratings "rapidly" step to the steady level of decay before they are utilized in practice. Essentially, the accelerated aging approach removes the most unstable part of the UV-induced index change, thereby stabilizing the grating. 


\subsubsection{Thermal stability models of FBGs}

By observing the long time evolution of erbium-doped germanosilicate FBGs at various high temperatures, Erdogan has shown that the thermal decay of a grating in its refractive index modulation could be characterized by a "power law" model [15]. A variant method of the above model, called "master aging curve" model was also proposed to simultaneously analyze all the thermal decay curves corresponding to different temperatures, and it was developed as a more general model by Kannan [16]. However, the Erdogan power law model was reported to be not applicable in the case of hydrogenloaded FBGs, and it was suggested to follow either a log-time model [17] or a simpler power law model [18]. Finally, deviations of demarcation energy distributions from Erdogan's models have been found in deuterium-loaded single mode fiber Bragg grating by applying the master aging curve model to their thermal annealing data [19-20].

It has been noted that every grating decay model could only describe the thermal decay behavior of some specific gratings. The thermal stability of FBGs is dependent on several factors such as dopants, hydrogen-loaded or unloaded, and finally grating writing conditions (including UV writing wavelength, pulsed or continuous wave, the fluence per pulse, etc.). Since the underlying mechanism of thermally induced refractive index decay is too complex to understand, a good model of grating thermal decay is difficult to develop.

\subsubsection{Thermal stabilization by Pre- and Post-exposure techniques}

Some researches found that the thermal stability of grating reflectivity could be improved by the pre- or post-exposure techniques [21-23]. The pre- or post-exposure technique 
includes an additional blanket exposure of UV light on the fiber either before or after the grating is formed inside the fiber. Exposure with uniform UV laser beam results in a large DC index change induced in the grating, and it is demonstrated that this elevated DC index change can make the thermal degradation of the $\mathrm{AC}$ index change smaller, hence thermally stabilizing the grating reflectivity. This approach has been proven effective for both hydrogen loaded and unloaded optical fiber in the above references.

\subsection{Outline of the Thesis}

Besides the traditional approach of stabilizing the grating structure by annealing treatment, the pre- or post-exposure techniques provide an additional way to improve the thermal stability of gratings. However, these techniques involve a two-step procedure, which makes the fabrication of gratings more complex. In this thesis, we attempt to develop a single-step procedure to inscribe gratings with low index contrast (namely, elevated DC refractive index change). Taking advantage of the limited spatial coherence of our excimer laser UV beam, lower contrast refractive index change patterns are obtained by increasing the fiber/phase mask distance. Thermal stability of gratings with different index contrast will be investigated. Another goal is to explore whether small differences in fiber/mask distance during volume manufacturing of FBGs would impact the thermal stability.

Following this introduction, Chapter 2 describes some theoretical background on the thermal stability of FBGs. It briefly introduces the spectral property of a uniform fiber Bragg grating, and the impact of fiber/phase mask distance on the grating index contrast. 
Furthermore, several existing models characterizing thermal decay of fiber Bragg gratings are also reviewed.

Chapter 3 mainly focuses on the experimental techniques, including the experiment setup of the fiber Bragg grating inscription, and the accelerated aging test setup designed for multiple gratings. In this chapter, the typical transmission and reflection spectra of fiber Bragg grating fabricated in hydrogen loaded single mode fiber is presented, and the grating growth behaviors both in DC and AC refractive index change are analyzed. Some detailed methodological problems concerning the accelerated aging test and proper solutions are addressed.

Chapter 4 describes the isothermal annealing experiments on two groups of gratings, which have different grating index modulation and are fabricated at various fiber/phase mask distances. The thermal decay curves of these hydrogen loaded pre-annealed gratings are presented, and the simple power decay model fitting results are provided.

Chapter 5 discusses the dependence of the grating stability on some parameters such as the grating strength and grating index contrast based on the accelerated aging experiment results. The validity of the existing grating decay models is also discussed. Finally, a comparison between our experiment and other experiments in literature is done, and a possible mechanism for the enhancement of a DC component on the thermal stability of grating reflectivity is suggested.

Chapter 6 summarizes the conclusions based on the results of this thesis and suggests some directions for future work on the subject. 


\section{Chapter 2}

\section{Background Theory}

This chapter will introduce the spectral properties of a uniform fiber Bragg grating, which are well characterized by the coupled mode theory. In the second part of this chapter, the issue of thermal stability of gratings will be addressed, and several existing models on thermal decay of fiber Bragg gratings will be reviewed. With the use of a proper decay model characterizing the decay of a grating, one can make a prediction on its lifetime at normal operating temperatures (less than $70^{\circ} \mathrm{C}$ ).

\subsection{FBG Spectral Characteristics}

\subsubsection{Spectral Shape}

A fiber Bragg grating consists of a periodic modulation of the refractive index in the core of a single-mode optical fiber, where the phase fronts are perpendicular to the fiber's longitudinal axis. Due to the refractive index variation inside the fiber, each periodic unit of a fiber Bragg grating could be considered as a mirror (optic thin film) to reflect light guided along the core of an optical fiber. Hence, the fiber Bragg grating, as an entity of numerous successive grating planes, is analogous to a volume hologram grating or a crystal lattice diffracting X-rays, in which Bragg diffraction was firstly discovered. If the wavelength of incident light satisfies the Bragg condition, the reflected light from each of the subsequent grating planes interferes constructively in the backward direction, and as a result the light of this wavelength is reflected by the fiber Bragg grating. Light of 
wavelengths that do not satisfy the Bragg condition will get transmitted through the fiber since the reflected light from each grating plane becomes progressively out of phase and will eventually cancel out.

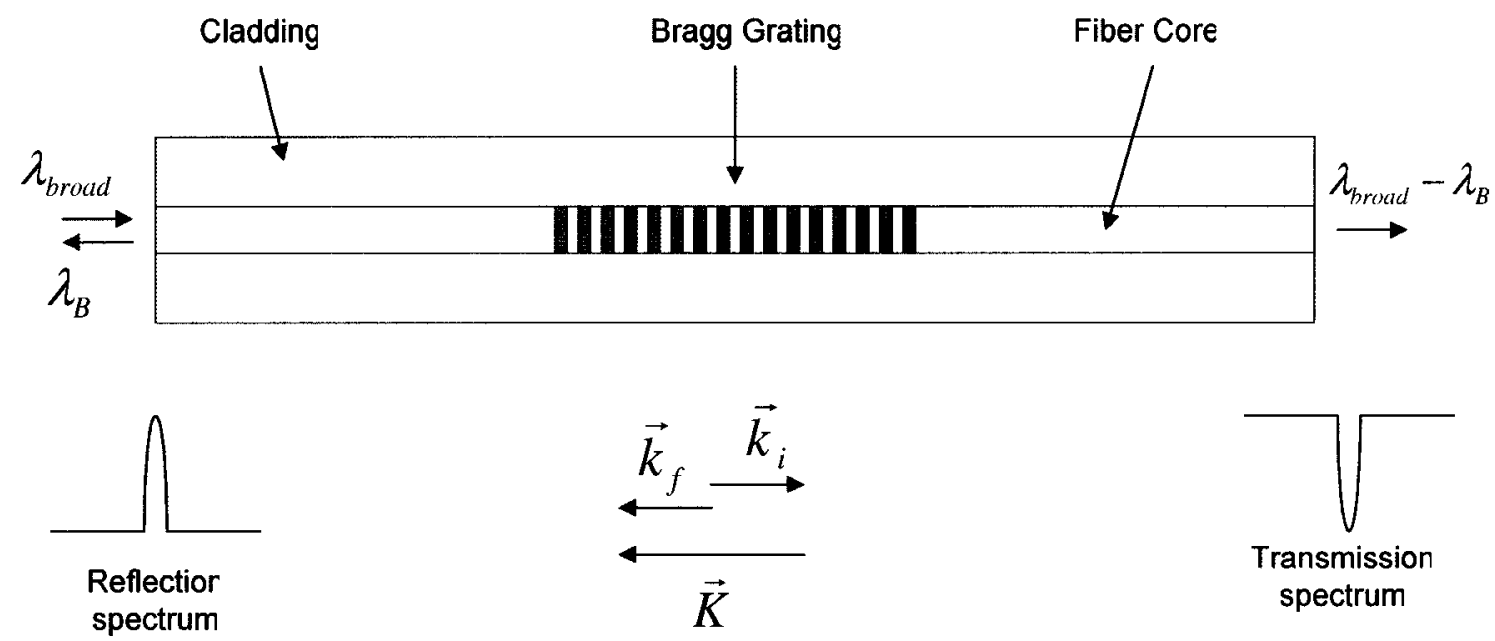

Fig 2.1 Schematic of a uniform Bragg grating with constant index of modulation amplitude and period [2]

Fig 2.1 illustrates the simplest uniform Bragg grating, which has a constant grating period as well as a constant modulation of the refractive index. A fiber Bragg grating functions as an optical filter, which will reflect the light at characteristic wavelength out of a broadband incident light. The characteristic wavelength, called Bragg wavelength, is determined by momentum conservation of the Bragg condition, namely that the summation of the incident wavevector $\vec{k}_{i}$ and the grating wavevector $\vec{K}$ is equal to the wavevector of the scattered radiation $\vec{k}_{f}$ :

$$
\vec{k}_{i}+\vec{K}=\vec{k}_{f}
$$

Since the incident and reflected lightwaves counter propagate inside the fiber, $\vec{k}_{i}$ and $\vec{k}_{f}$ should be the propagating constants of the fiber modes for forward and backward fields, 
which have same magnitude of $2 \pi n_{e f f} / \lambda_{B}$ but opposite directions. For a first order Bragg grating, the grating wavevector $\vec{K}$ has a magnitude of $2 \pi / \Lambda$ and a direction normal to the grating planes. With above conditions, the Bragg condition is simplified as:

$$
\lambda_{B}=2 n_{e f f} \Lambda
$$

where $\lambda_{B}$ is the Bragg wavelength, $n_{e f f}$ is the effective refractive index for the fiber mode at the Bragg wavelength, and $\Lambda$ is the grating period.

In addition to changing the grating period, the grating's refractive index profile can also be used to modify the reflectivity and the spectral shape of the grating. Assuming that the refractive index change distributes uniformly across the fiber core and does not exist in the outside region, the refractive index profile of a uniform fiber Bragg grating has a sinusoidal modulation form as

$$
n(z)=n_{0}+\Delta n_{m o d} \cos \left(\frac{2 \pi z}{\Lambda}\right)
$$

where $n(z)$ is the refractive index of a grating inside the fiber core, $n_{0}$ is the refractive index of the unperturbed fiber core, $\Delta n_{\text {mod }}$ is the refractive index modulation amplitude, and $z$ is the distance along the fiber longitudinal axis. According to the coupled mode theory, the reflection properties of a Bragg grating are regarded as light coupling between counter propagating optical fields guided inside the grating and the reflectivity of a uniform fiber Bragg grating is easily calculated from the coupling equations [24-25] as:

$$
R(l, \lambda)=\frac{\kappa^{2} \sinh ^{2}(s l)}{\Delta k^{2} \sinh ^{2}(s l)+s^{2} \cosh ^{2}(s l)}
$$


where $R$ is the power reflectivity of the grating, $l$ is the grating length, $\lambda$ is the reflected wavelength, $\kappa$ is the coupling coefficient, $\Delta k=k-\frac{\pi}{\Lambda}$ is the detuning wavevector (which is the difference between propagation constant at reflected wavelength and that at Bragg wavelength), and $s^{2}=\kappa^{2}-\Delta k^{2}$. Note that the propagation constant $k$ is equal to $2 \pi n_{e f f} / \lambda$, where the effective index $n_{e f f}$ is not the average fiber core refractive index $n_{0}$ in the Eq. (2.3) but can be determined from $n_{0}$. The coupling coefficient $\kappa$ is given by

$$
\kappa=\frac{\pi \Delta n_{m o d}}{\lambda} \Gamma
$$

where the confinement factor $\Gamma$ is the fraction of the fiber mode power contained by the fiber core. $\Gamma$ is approximately 0.75 for the fundamental mode of a single mode fiber in the wavelength region around $1550 \mathrm{~nm}$. The simulated reflection spectrum of a typical uniform fiber Bragg grating is shown in Fig 2.2. The maximum reflectivity occurs at the Bragg wavelength, which corresponds to a detuning wavevector of zero. Besides the main reflectance peak of the grating, there are several small side lobes situated symmetrically on both sides of the Bragg resonance. For applications that place high demands on the spectral shape of the grating, a technology called apodization can be incorporated into the process of fiber Bragg grating inscription to suppress these undesirable side lobes. 


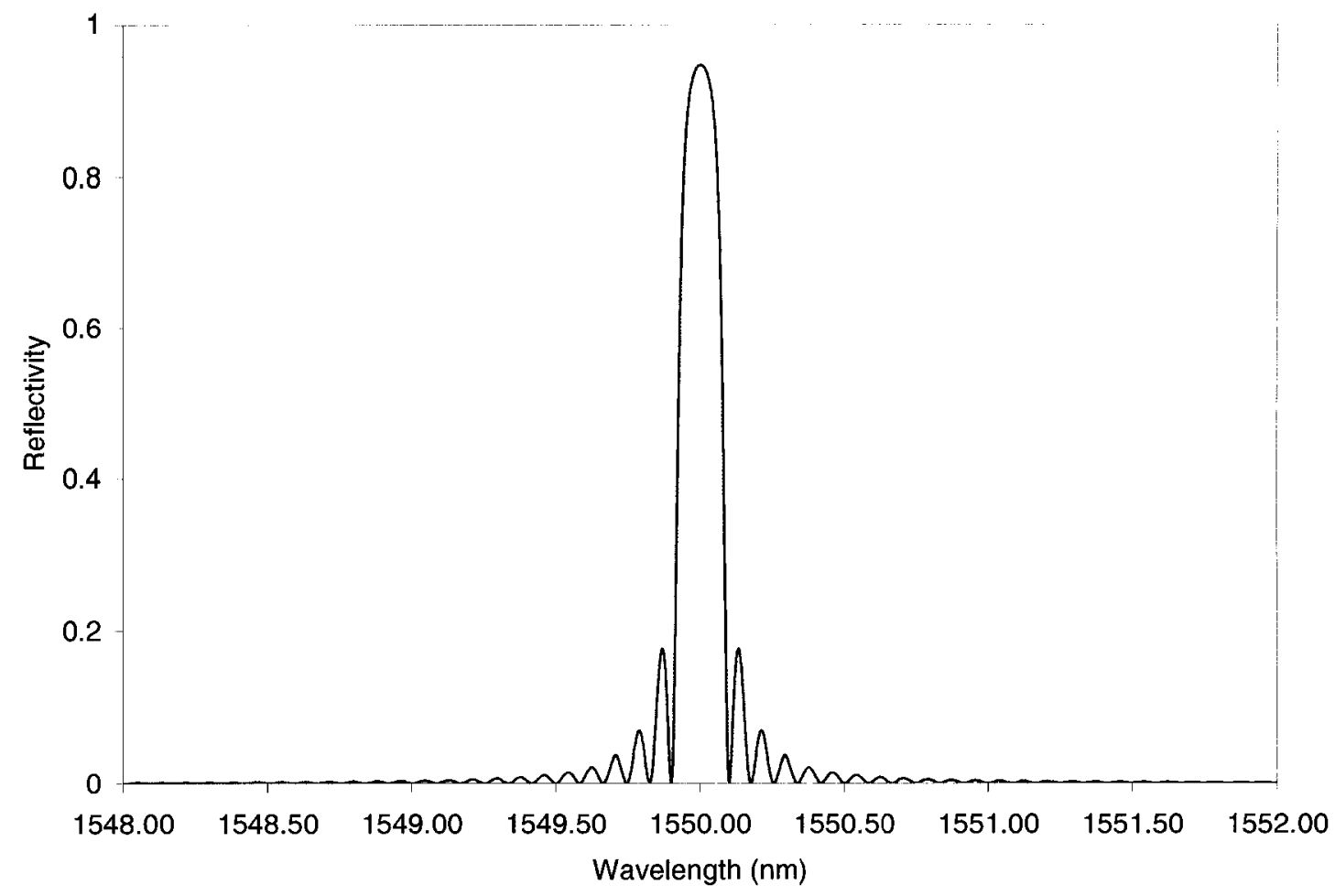

Fig 2.2 A typical simulated reflection spectrum of a uniform fiber Bragg grating

\subsubsection{Formula for $\mathrm{DC}$ and $\mathrm{AC}$ index change}

In the above discussion, the index perturbation of a grating is assumed simply as a sinusoidal modulation without any average change imposed on the fiber, which has the form of Eq. (2.3). As a result, the Bragg wavelength $\lambda_{B}$ corresponding to the maximum reflectivity of fiber Bragg grating will be fixed at a single value of $2 n_{\text {eff }} \Lambda$ no matter how strong a grating is formed inside the fiber. However, in practice, it has been observed that the Bragg wavelength of a grating evolves as a function of the inscribing time during the grating fabrication. This shift of Bragg wavelength during the fabrication is caused by the change in the effective refractive index in the grating region, which means that a DC refractive index change is introduced into the grating in addition to an $\mathrm{AC}$ refractive index change. 
The core index profile of a grating that has both $\mathrm{DC}$ and $\mathrm{AC}$ index changes can be written in a more complex form:

$$
n(z)=n_{0}+\Delta n_{\text {mean }}+\Delta n_{\text {mod }} \cos \left(\frac{2 \pi z}{\Lambda}\right)
$$

where $\Delta n_{\text {mean }}$ is the average refractive index change in the fiber core. Other variables in the above equation are same as those in Eq. (2.3): $n_{0}$ is the refractive index of the unperturbed fiber core, $\Delta n_{\text {mod }}$ is the refractive index modulation amplitude.

Accordingly, the refractive index perturbation of the fiber core results in the following form of the effective index of the guided modes:

$$
n_{e f f}(z)=n_{e f f}^{0}+\Delta n_{e f f}^{\text {mean }}+\Delta n_{e f f}^{\text {mod }} \cos \left(\frac{2 \pi z}{\Lambda}\right)
$$

where $n_{\text {eff }}^{0}$ is the effective refractive index of the unperturbed fiber which has core index of $n_{0}$. Furthermore, $\Delta n_{\text {eff }}^{\text {mean }}$ and $\Delta n_{\text {eff }}^{\text {mod }}$ are the DC and AC components of the effective refractive index change respectively.

Assuming that the index perturbation of the grating is uniform across the fiber core and not in the cladding, which is often true due to the lack of photosensitivity of the cladding, the change in effective refractive index will be approximately proportional to a change in the core index when the latter varies by a small amount (below $1 \times 10^{-3}$ ) [26]. Mathematically, the variables in the Eq. (2.6) and Eq. (2.7) follow the relationship as:

$$
\begin{aligned}
& \Delta n_{\text {eff }}^{\text {mean }}=\Delta n_{\text {mean }} \cdot \Gamma \\
& \Delta n_{\text {eff }}^{\text {mod }}=\Delta n_{\text {mod }} \cdot \Gamma
\end{aligned}
$$

where $\Gamma$ is the confinement factor of the guided mode. 
As previously mentioned, the shift of Bragg wavelength during the formation of a grating is related to the DC component of the refractive index perturbation. Let $\lambda_{B, 0}$ be the Bragg wavelength at the beginning of the fabrication of the grating written at the pristine fiber, and $\lambda_{B}$ be the Bragg wavelength of the formed grating. According to the Bragg condition, the Bragg wavelengths $\lambda_{B, 0}$ and $\lambda_{B}$ correspond to their own grating's average effective refractive index $n_{\text {eff }}^{0}$ and $n_{e f f}^{0}+\Delta n_{\text {eff }}^{\text {mean }}$, respectively. Consequently, the DC effective index change of a grating $\Delta n_{e f f}^{\text {mean }}$ is given by:

$$
\Delta n_{\text {eff }}^{\text {mean }}=\frac{\lambda_{B}-\lambda_{B, 0}}{2 \Lambda}=\frac{\Delta \lambda_{B}}{2 \Lambda}
$$

where $\Delta \lambda_{B}$ is the Bragg wavelength shift, and $\Lambda$ is the grating pitch. Based on the Eq. (2.8), the DC refractive index change of a grating can be expressed as:

$$
\Delta n_{\text {mean }}=\frac{\Delta \lambda_{B}}{2 \Lambda \Gamma}
$$

Generally, when referring to the index perturbation of a grating, it is more common to use the $\mathrm{DC}$ refractive index change in the fiber core rather than the $\mathrm{DC}$ effective refractive index change.

The AC component of refractive index change for a FBG can be evaluated from the reflectivity of the grating. The Eq. (2.4) shows that the reflectivity of the grating achieves its maximum at the Bragg wavelength, and the peak reflectivity is given by:

$$
R_{\max }=\tanh ^{2}(\boldsymbol{\alpha})
$$

By substituting the expression of the coupling coefficient $\kappa(\mathrm{Eq}(2.5))$ into the above equation, the refractive index modulation amplitude in the fiber core is given by 


$$
\Delta n_{\text {mod }}=\frac{\lambda_{B} \cdot \tanh ^{-1}\left(\sqrt{R_{\max }}\right)}{\pi \cdot \Gamma \cdot l}
$$

Its counterpart in terms of effective refractive index is easily expressed as:

$$
\Delta n_{e f f}^{\text {mod }}=\Delta n_{m o d} \cdot \Gamma=\frac{\lambda_{B} \cdot \tanh ^{-1}\left(\sqrt{R_{\max }}\right)}{\pi \cdot l}
$$

\subsubsection{Influence of fiber/phase mask distance (writing distance) on contrast}

As mentioned in the last subsection, the fabrication of a FBG in practice involves introducing both DC and AC refractive index change inside the fiber core, although only AC component contributes to the grating reflectivity and is necessary for forming a grating. The refractive index change is induced by exposing the fiber to UV light, which is referred to as photosensitivity of the fiber. Furthermore, the grating profile of UV induced refractive index change could be achieved by using spatial modulation of UV beam, which is an interference pattern of a UV beam produced by employing either interferometric technique or phase mask. As the UV induced refractive index change is directly dependent on the interference fringes, the coherence of the UV beam plays an important role on the quality of the formed grating, which is evaluated by the grating contrast (the ratio of $\mathrm{AC}$ to $\mathrm{DC}$ refractive index change). We will only discuss the case where the phase mask technique is employed in our grating fabrication experiment.

A phase mask, which is a silica plate with periodic etched grooves on one surface, acts as a diffractive grating to diffract the incident light beam in some specific angular directions denoted as diffracted orders $m$ ( $m$ is an integer, $m=0, \pm 1, \ldots$ ). The interference fringes utilized to imprint the grating are produced by the plus and the minus first order diffracted beams of the phase mask, which is typically designed to suppress the 
diffraction efficiency of the zeroth order diffracted beam to less than $3 \%$. To manufacture a fiber Bragg grating, the optical fiber is placed in near contact with the phase mask as shown in Fig 2.3.

UV laser beam

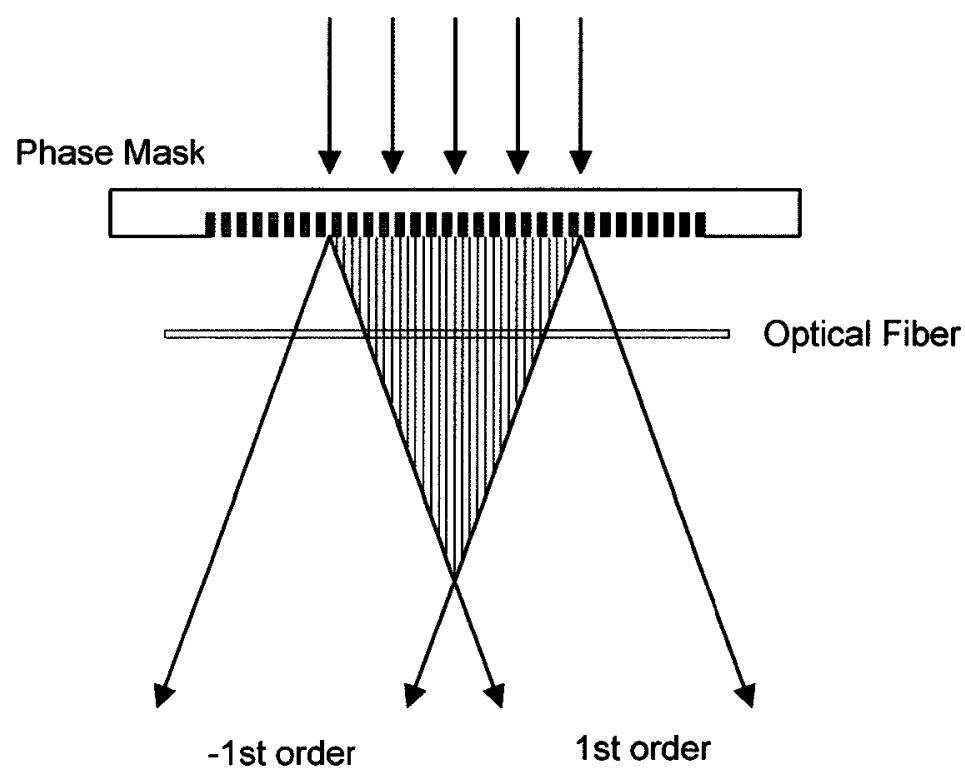

Fig 2.3 Schematic of the phase mask technique for inscribing fiber Bragg gratings

Considering the scheme more deliberately, the interfering arms of the fringe pattern are the plus and minus first diffracted orders emerged from different parts of the phase mask, which are located symmetrically about the target interference point. Due to the identical distance of two interfering arms from the phase mask to the fiber, the temporal coherence requirement of the incident beam to obtain a high contrast fringe pattern is relaxed.

However, the spatial coherence of the incident beam plays a critical role in the quality of interference fringe pattern. If the fiber is placed near contact with the phase mask, the interfering beams emerging from two close parts of the phase mask have high degree of correlation, and as a result the maximum contrast in the induced refractive index change 
is achieved. In contrast, if the distance of the fiber from the phase mask is sufficiently large such that the corresponding separation between two interfering beams emerging from the mask becomes beyond the spatial coherence of the incident beam, no interference will occur since interference beams are completely incoherent. It is clear that the contrast of interference fringe pattern and therefore that of the formed grating can be controlled by the fiber/phase mask distance. As the distance increases, the gradual loss of spatial coherence between the plus and minus first orders destroys the interference, and the grating contrast diminishes to zero accordingly.

More detailed mathematical analysis on the near field diffraction pattern from a phase mask has been done in the reference [27], in which the spatial coherence of incident UV beam was included as an important parameter affecting the diffraction pattern. In practice, the fiber is usually put directly behind the phase mask in order to fabricate a fiber Bragg grating with the best quality. On the other hand, the writing distance of the fiber from the phase mask provides an easy way to control the grating contrast of refractive index, which is employed in the following research.

\subsection{Thermal Stability Models of Fiber Bragg Gratings}

\subsubsection{Erdogan power model}

Erdogan et al. [15] conducted the first thorough investigation on the thermal decay of fiber Bragg gratings and proposed an empirical model to characterize it, which has been widely used in the following work on this issue. In their experiment, gratings fabricated within erbium-doped germanosilicate fibers were subjected to thermal annealing at different elevated temperatures. They quantify the thermal decay of grating strength by a 
grating parameter called integrated coupling constant (ICC) rather than the peak reflectivity of grating. The ICC is proportional to the UV-induced refractive index modulation of gratings. According to the coupling mode theory, the ICC of a Bragg grating is given by:

$$
I C C=\tanh ^{-1}\left(\sqrt{1-T_{\min }}\right)
$$

where $T_{\min }$ is the minimum transmission of grating at Bragg wavelength. For convenience, the ICC is generally normalized to its initial value at the beginning of the annealing experiment, and this new quantity is called the normalized ICC (NICC).

Fig 2.4 shows the thermal degradations of gratings at temperature of $350{ }^{\circ} \mathrm{C}$ and $550{ }^{\circ} \mathrm{C}$. As shown in this figure, each grating exhibits an initial sharp decrease in its NICC, followed by a progressively slower decay with non-zero rate of change. The decay of the grating could be described by the power law function:

$$
\eta=\frac{1}{1+A\left(t / t_{1}\right)^{\alpha}}
$$

where $\eta$ is the NICC of the grating (i.e. the normalized refractive index change, due to the proportionality of ICC to refractive index change), $t$ is the time, and $t_{1}=1 \mathrm{~min}$ or $1 \mathrm{~s}$ is used to keep dimensions consistent. The factors $A$ and $\alpha$ are dimensionless, and both of them are dependent on temperature.

It is very important to investigate the relationships between the decay factors $A$ and $\alpha$ and the heating temperature, because these relationships can be used to determine the thermal decay pattern of a grating at a given temperature. Based on the analysis of experiment data obtained at various heating temperatures, $A$ and $\alpha$ are plotted against 


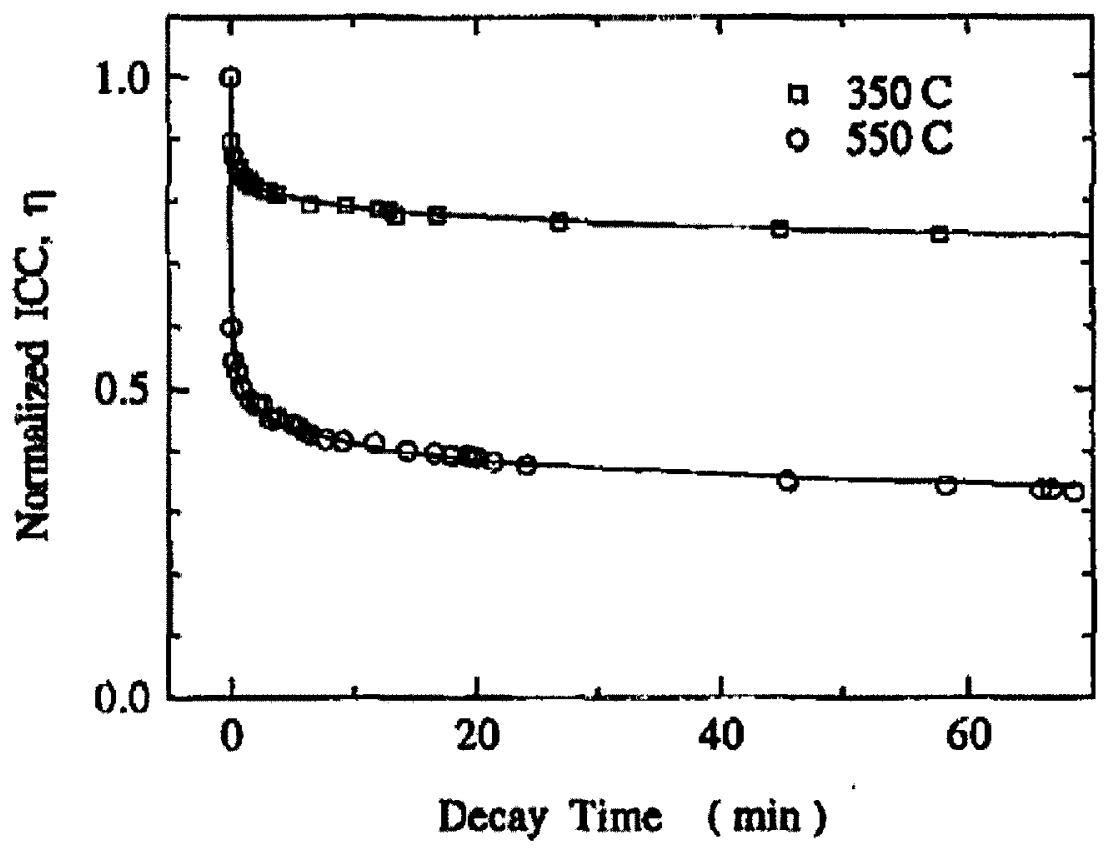

Fig 2.4 Thermal decay of normalized ICC for two gratings as a function of decay time at temperature of $350^{\circ} \mathrm{C}$ and $550^{\circ} \mathrm{C}$ [15]
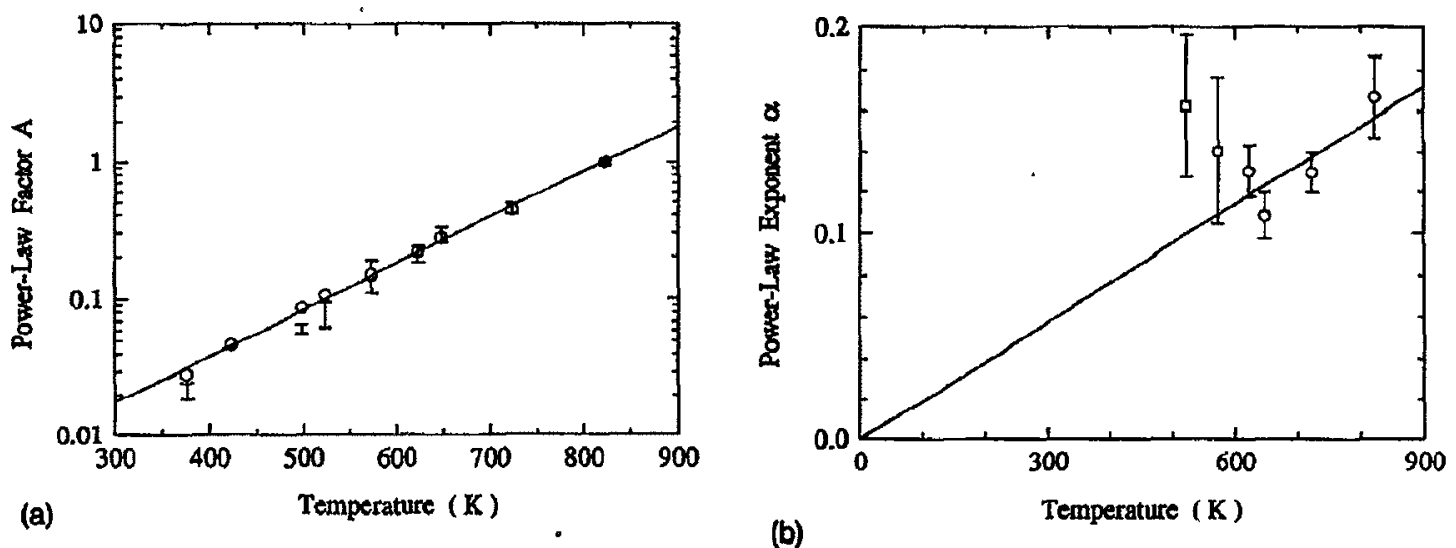

Fig 2.5 (a) The power law factor $A$ varying as a function of temperature (b) The power law exponent factor $\alpha$ varying as a function of temperature [15] 
temperature to evaluate their temperature dependence. These plots are illustrated in Fig 2.5(a) and Fig 2.5(b) respectively. In Fig 2.5(a), it can be seen that the logarithm of $A$ follows a linear relationship with the temperature quite well, therefore the power law factor $A$ has the form:

$$
A=A_{0} \exp (a T)
$$

where $T$ is the decay temperature measured in Kelvin, $A_{0}$ and $a$ are independent constants for a given grating. In Fig 2.5(b), the power exponent $\alpha$ is found to follow a possible linear dependence on the temperature:

$$
\alpha=\frac{T}{T_{0}}
$$

with the temperature measured in Kelvin. $T_{0}$ is also a constant for a given grating.

As the power law model proposed above is completely empirical arising from fitting analysis of experiment data, its accuracy and reliability in describing the thermal decay behavior of gratings is still a concern. Erdogan's power law model could represent the thermal degradation at each single elevated temperature quite well, as shown in the Fig 2.4. In addition, it is acceptable that the power law factor $A$ follows an exponential function of the temperature since Eq. (2.17) apparently fits the experiment data very well in the Fig 2.5(a). However, the correctness of the dependence of factors $\alpha$ on the temperature is still uncertain. Although the variation of $\alpha$ as a function of temperature displays a linear relationship for temperature greater than $600 \mathrm{~K}$, there is a substantial deviation between the assumed linear fit line and the obtained experiment data at lower temperatures. Even though this large error was interpreted as arising from the large uncertainty in the measurement of ICC which decays substantially less at lower 
temperature, the validity of extrapolating thermal decay of gratings at much lower temperature (i.e. typical device operating temperature) through the temperature dependence of factors $A$ and $\alpha$ is unknown due to the existence of this error. This issue will be investigated in depth in our research.

Erdogan's power model was used extensively in subsequent publications studying the thermal decay of FBGs written on different fibers [28-34], and it has been shown that Erdogan's model depicts the thermal decay behavior for those different gratings quite well. Again, in some publications there is still evidence that the power exponent $\alpha$ does not follow a linear relationship with the temperature at low temperatures. Therefore, Erdogan's power model is only an empirical model with possible invalidity on thermal decay at lower heating temperature.

\subsubsection{Master aging curve model}

In the same paper, Erdogan proposed an alternative approach, which is based on a simple interpretation on the physical mechanism underlying thermal decay of gratings, to analyze the accelerated aging data at various temperatures. That approach is called the master aging curve model, which was widely used as a more general model in the following research $[16,32-35]$.

In this theoretical picture, the formation of a grating involved the excitation of carriers (e.g., electrons) from the defect energy state. These free carriers are trapped in a broad distribution of energy states rather than a single trap level considering the inherent amorphous property of glass. Intuitively, thermal erasure of a grating is assumed as a reversal process of the UV-induced refractive index change. The carriers are thermally 
excited from their trapping sites and repopulate their original energy state again, and their contribution to the UV-induced refractive index change is lost. Fig 2.6 shows the energy diagram of the above simple mechanism. Furthermore, the detrapping rate at any energy state is dependent on the heating temperature and the erasure activation energy, which is the energy gap between the trap energy state and the conduction band. It is this erasure activation energy that should be overcome for a carrier transiting back to its original state. The trapped sites with lower activation energy decay faster than those with higher activation energy. A physical quantity called demarcation energy $E_{d}$ has been defined to describe the advancement of the carrier excitation process. For a thermal decay at a given temperature and time, approximately all carriers residing at shallower traps with activation energy less than the demarcation energy $E_{d}$ would be evacuated, but carriers in deeper traps remain filled. The remaining UV-induced refractive index change is proportional to the population of these deeper traps.

Based on the above discussion, master curve model suggest exploring the thermal degradation of gratings in a new way, in which the extent of aging on grating is described by the demarcation energy $E_{d}$ instead of the heating time $t$ as in the power law model. Experimental data sets obtained from thermal decay at multiple temperatures are grouped together to plot a composite curve, which is called master curve representing the evolution of NICC against the demarcation energy $E_{d}$. An example of master curve is shown in Fig 2.7, where different symbols correspond to the different thermal decay at various temperatures. In order to keep mathematical consistence with the empirical power-law model, the NICC of a grating should have the form: 


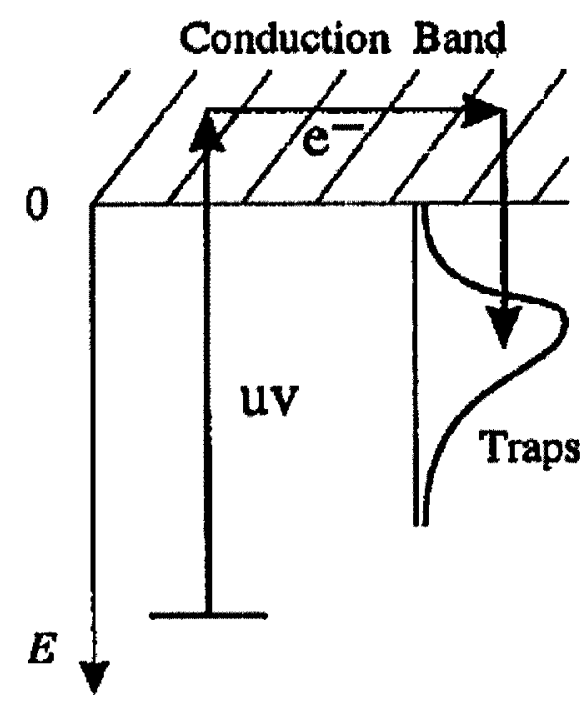

(a)

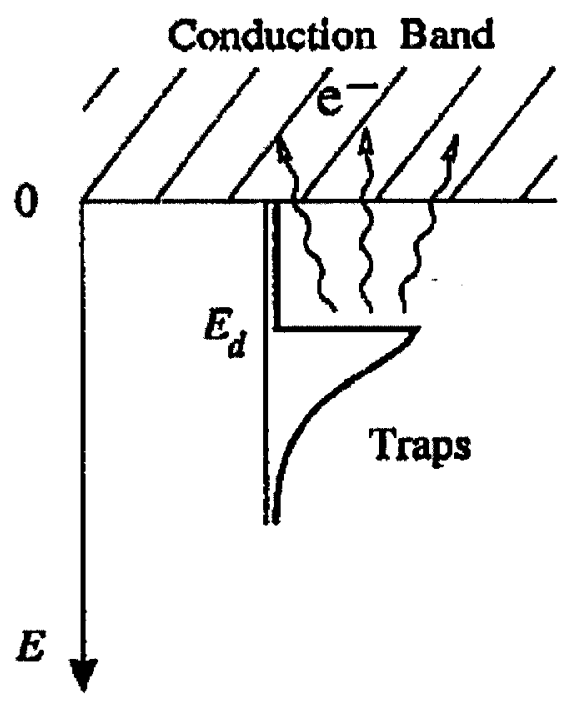

(b)

Fig 2.6 Diagram of the physical model for (a) electrons excited by UV irradiation during grating formation, and (b) thermal depopulation of traps during thermal decay of a grating [15]

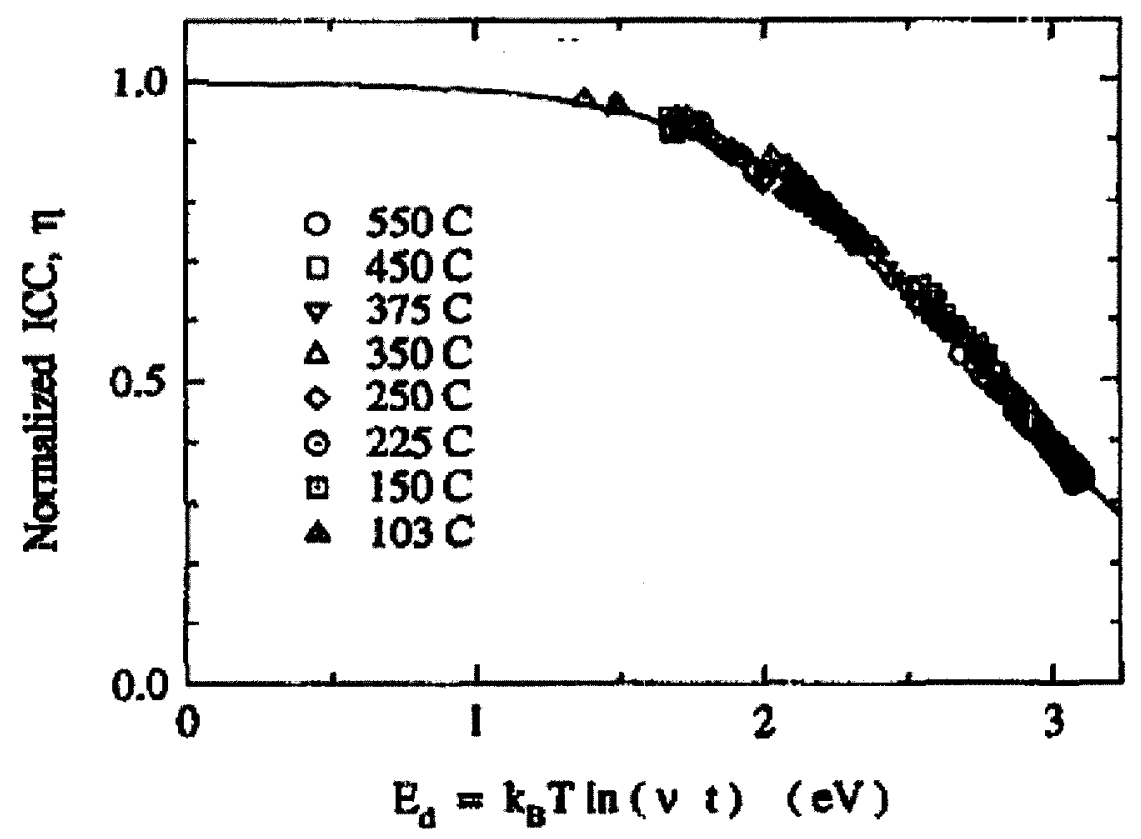

Fig 2.7 Plot of the NICC as a function of the demarcation energy $E_{d}$ [15] 


$$
\begin{aligned}
& \eta=\frac{1}{1+\exp \left[\left(E_{d}-E_{0}\right) / \Delta E\right]} \\
& E_{d}=K_{B} T \ln (v t)
\end{aligned}
$$

where $K_{B}$ is the Boltzmann constant, $T$ is the decay temperature, $t$ is the decay time, and $v$ is the attempt frequency, which is a fitting factor obtained by forcing the datasets at various temperatures to merge together on a single curve through an iterative process. $E_{0}$ and $\Delta E$ are fitting factors which are obtained by fitting Eq. (2.19) to the master curve.

A closer inspection of Eq. (2.19) and Eq. (2.16) reveals that master aging curve model is merely a mathematical variant of the empirical power law model, and the fitting factors can be easily converted to each other as:

$$
\begin{aligned}
& E_{0}=-K_{B} T_{0} \ln \left(A_{0}\right) \\
& \Delta E=K_{B} T_{0} \\
& v=\exp \left(a T_{0}\right)
\end{aligned}
$$

From the point view of data analysis, master curve model shows advantage over empirical power law model since the former fits all the experimental data sets together and thus will obtain more accurate results than the latter, which fits decay data sets individually. However, although master aging curve model is based on theoretical interpretation of thermal decay, it is still only an empirical model since the real physical mechanism is more complex and may conflict with such explanation. Apparently, the validity of this model will be challenged if multiple decay data sets at different temperatures can not be collapsed onto a single curve. 


\subsubsection{Log time model}

Although Erdogan's power law model has been widely used to represent the thermal decay of nonhydrogen loaded FBGs written in different fiber materials, Baker et al [17] found that it was not applicable for the case of hydrogen loaded FBGs. Hydrogen loading is an effective solution to enhance the photosensitivity of an optical fiber [36], but hydrogen loaded fiber Bragg gratings are less stable than those written in fibers without the assistance of hydrogen.

Baker has investigated thermal degradation of hydrogen loaded gratings written in boron and germanium codoped fiber. Prior to starting thermal aging experiments, the gratings are pre-annealed at $85^{\circ} \mathrm{C}$ for 48 hours in order to diffuse out the remaining gaseous molecular hydrogen inside the fiber, and the less stable portion up to $20 \%$ of UV-induced index modulation is removed simultaneously. Experimental data obtained at different temperatures are illustrated in Fig 2.8. In this figure, when a log time scale is used, the decay slope of the NICC is constant for all temperatures, and the decay does not begin until a certain time, which dependents on the temperature. This evidence indicates that the NICC of a hydrogen loaded fiber grating should be expressed as 


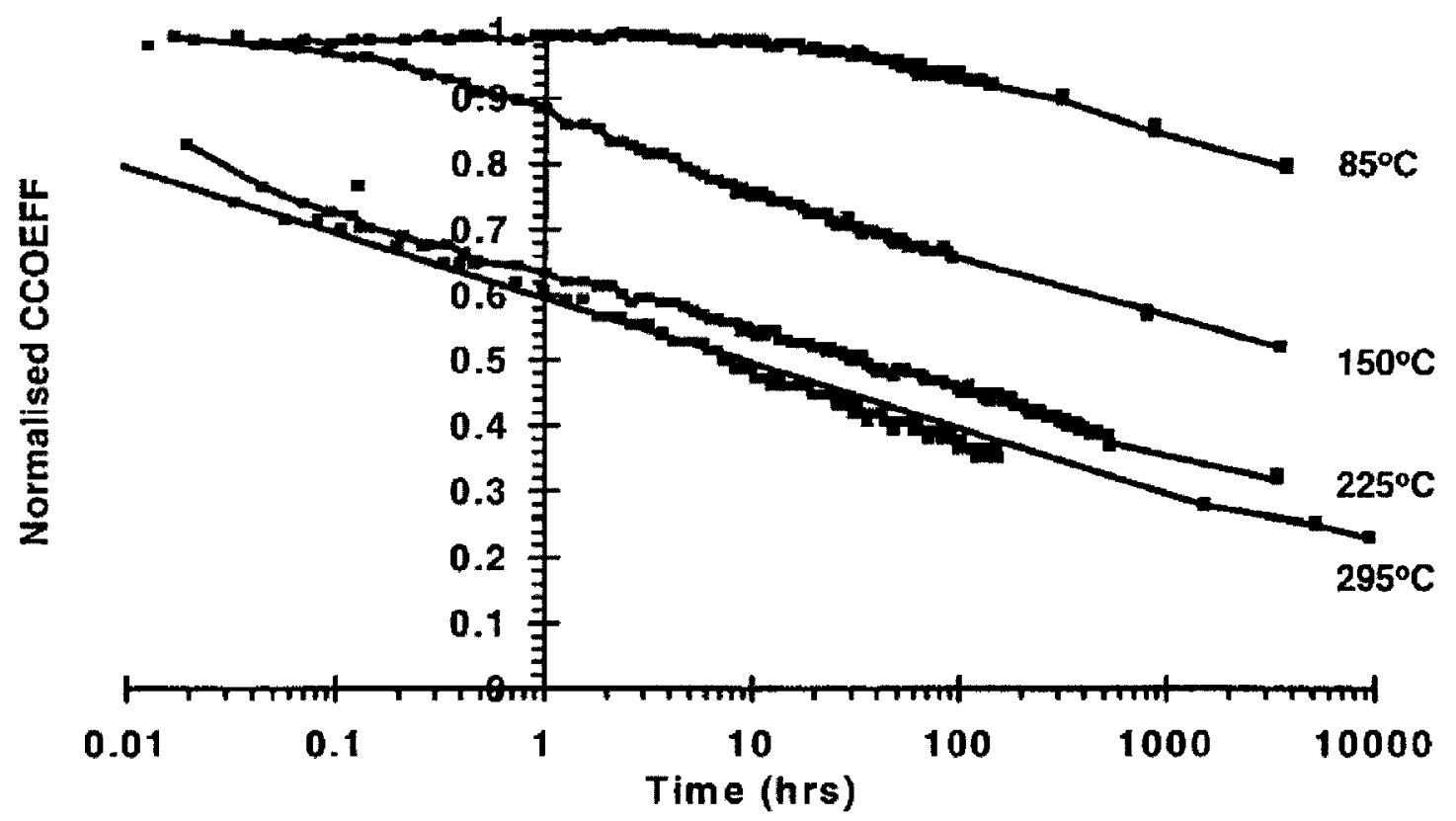

Fig 2.8 Plot of the NICC of a hydrogen loaded fiber grating as a function of time [17]

$$
\eta= \begin{cases}1-k \log (t / \tau) & \text { for } t>\tau \\ 1 & \text { for } t \leq \tau\end{cases}
$$

where $k$ is a constant, and $\tau$ is the start time of the decay, which is temperature dependent. Examining the temperature dependence of $\tau$, it is found that:

$$
\tau=A \exp (B / T)
$$

where $A$ and $B$ are independent constants.

\subsubsection{Other models}

Observing different thermal decay behavior of hydrogenated fiber Bragg gratings from the log time model, Ishikawa demonstrated experimentally that a simple power model could depict the decay property of hydrogen loaded fiber Bragg gratings quite well [18].

$$
\eta= \begin{cases}a t^{b} & \text { for } t>\tau=a^{-1 / b} \\ 1 & \text { for } t \leq \tau=a^{-1 / b}\end{cases}
$$


Also, the temperature dependence of its fitting factors has been well developed for determining the decay at any temperature and time phase.

Another decay model called the variable reaction pathway (VAREPA) approach was proposed by Poumellec [37], which takes a similar mathematical procedure to analyze the decay data as the master aging curve model. The difference is that its interpretation of the distribution of activation energy originates from the different reaction pathways instead of different energy levels of trapped sites.

\subsection{Aging model used in this thesis}

Many aging models have been proposed to describe the thermal decay characteristics of fiber Bragg gratings, but all of them are only correct to some extent. Erdogan's power law model has some uncertainty on the temperature dependence of power exponent factor, and it is only good for the nonhydrogen loaded gratings. Master curve model will turn out to be unreliable if accelerated aging data at various temperatures could not overlap with each other no matter what value is used for the attempt frequency. As for the hydrogenated fiber Bragg gratings, log time model and simple power law model obviously demonstrate two different thermal decay behaviors. Therefore, the thermal degradation of fiber Bragg gratings is a rather complex phenomenon, which is difficult to explain with a generally applicable description either mathematically or physically. Thermal decay in UV-induced index change could be dependent on many factors, such as the type of fiber material, with or without hydrogen loading, grating writing conditions such as continuous wave or pulsed laser source, operating wavelength of laser source and the writing energy. 
Although a general model for the thermal decay of fiber Bragg gratings is absent, the common procedure, which those aging models take to deal with thermal experiment data, will be followed in our research. Without losing any generality, we will analyze the thermal experimental results using the following steps:

1. Plotting NICC versus time $t$ or $\log$ time $\ln (t)$

2. Finding a proper function which fits the thermal degradation profile

3. Developing the dependence of the fitting factors on the temperature

Once these analysis steps are completed, the thermal decay characteristics of a given grating will be well defined. 


\section{Chapter 3}

\section{Experimental Techniques}

This chapter will first introduce the grating fabrication setup in detail, followed by a discussion on the experimental results, including the typical spectra of a uniform FBG and the grating growth behaviors. Finally, the accelerated aging setup designed for observing the decay of multiple gratings simultaneously will be shown, and some methodological problems concerning the aging test and proper solutions will also be addressed.

\subsection{Grating Fabrication Set-up}

\subsubsection{Fiber description and Hydrogen loading technique}

The selection of fiber is the first important step in the manufacturing of a fiber Bragg grating. Typically photosensitive fiber employed to fabricate FBGs is doped with high concentration of germanium. However, it is exciting that hydrogen loading techniques has been found to enable us to introduce large refractive index change in a standard communication fiber (germanium concentration as low as $4.3 \% \mathrm{~mol}$ ) [36]. Due to its advantage of low cost, the standard communication fiber with the assistance of hydrogen loading is a routine way of manufacturing a fiber Bragg grating in industry. Hence, in order to replicate standard industrial practice, a standard telecom fiber (Coring SMF-28) will be utilized in our research. 
Hydrogen loading technique is a simple and effective method to enhance photosensitivity in optical fibers. It is generally carried out by immersing fibers into a high-pressure hydrogen atmosphere. For the sake of safety, a hydrogen loading chamber is specially designed by using an airtight metal tube with a small diameter, which minimizes the amount of hydrogen required to fill the chamber and hence the danger of hydrogen explosion. To load hydrogen, the Corning SMF-28 fiber is cut into short pieces (length $1 \mathrm{~m})$, and placed in the small tube, which is connected to a bottle of hydrogen gas via a valve controlling the gas filling process. After this procedure is settled down, fibers are maintained in the hydrogen tube at room temperature and pressure of $\sim 17.24 \mathrm{Mpa}$ (2500 psi) for about 14 days, which has been proven to be an appropriate duration enabling most of the hydrogen molecules to diffuse into the fiber core, reaching nearly saturation level in hydrogen concentration [38]. After hydrogen loading is finished, all hydrogenated fibers are stored in a refrigerator at about $-20{ }^{\circ} \mathrm{C}$ in order to prevent the hydrogen molecules from diffusing out of fibers.

\subsubsection{Irradiation}

The experiment setup of fabricating fiber Bragg gratings with phase mask technology is schematically illustrated in Fig 3.1. The fiber Bragg grating writing system consists of a laser source, beam shaping optics, a phase mask and a fiber mounting stage.

A switchable excimer laser (PulseMaster 840 series from GSI Lumonics), which can operate at the wavelength of $248 \mathrm{~nm}(\mathrm{KrF})$ or $193 \mathrm{~nm}(\mathrm{ArF})$, provides UV source for the inscription of the fiber Bragg gratings. For the application in hydrogen loaded fibers 
herein, $248 \mathrm{~nm}$ wavelength model is used. The pulse energy could achieve up to $400 \mathrm{~mJ}$ with pulse duration of around $20 \mathrm{~ns}$, and repetition rate can be varied up to $100 \mathrm{~Hz}$.

A slit is placed in front of the laser outlet in order to select the central uniform region of the laser beam. The slit dimensions are precisely adjusted to $7.630 \mathrm{~mm}$ by $2.724 \mathrm{~mm}$, which is only a small portion of the laser beam size. The slit blocks a large surrounding part of the UV light, which cannot be used to illuminate the fiber even with the presence of a focus lens. This arrangement also makes the alignment of the following optics elements easier.

Next, the laser beam is enlarged by a beam expander. It consists of two cylindrical lenses with focus lengths of $75 \mathrm{~mm}$ and $150 \mathrm{~mm}$ respectively. Passing through this component, the beam size will be magnified 2 times in the horizontal direction and unchanged in the vertical direction. This component makes the beam profile more uniform and increases the spatial coherence by a factor of two. It also acts as beam shaper to adjust the output laser, which is slightly divergent, to a beam of parallel light.

After the beam expander, another perpendicular cylindrical lens is used to focus the interference pattern along the fiber. Its focus length is $100 \mathrm{~mm}$. The incorporation of this focus lens obviously enables us make more efficient use of the laser beam, and typically in our setup the pulse fluence illuminating the fiber is improved about 20 times.

As a key part of fabrication system, the phase mask is mounted on a motion stage. The alignment of the phase mask is extremely important for inscribing a high quality fiber Bragg grating. All three rotational directions of the phase mask are provided by the motion stage in order to allow precise alignment of the phase mask perpendicular to the incident beam propagating axis. In addition, height adjustment of the phase mask is 
available to place it in the center of laser beam. The period of the phase mask used to write the FBGs is $1067.2 \mathrm{~nm}$. With the zeroth order diffracted beam suppressed by optimizing the design of the phase mask, the plus and the minus first order diffracted beams will form an interference fringe pattern with a period of $533.6 \mathrm{~nm}$. The interference fringes will photo-imprint a periodic pattern on a photosensitive fiber and a FBG with grating period of $533.6 \mathrm{~nm}$ will be formed. An aperture is placed right in front of the phase mask in order to adjust the width of the UV laser beam and hence the grating length of the formed FBG.

At last, a hydrogen loaded fiber is stripped about $2 \mathrm{~cm}$ in the center, and clamped between the two fiber holders of the fiber mounting stage. While the fiber is mounted, a small tension is applied to the fiber in order to keep it straight and therefore form a precise fringe pattern on the fiber. With the aid of the mounting stage, the fiber is aligned in all directions such that it is placed along the focused interference fringe. Moreover, the optical fiber is placed in close proximity to the phase mask, with the distance between the two typically around several tens of micrometers. The fiber mounting stage can precisely adjust this distance with an accuracy of $\sim 10 \mu \mathrm{m}$. This distance is an important parameter in controling the grating refractive index contrast, as discussed in chapter 2 . In the course of inscribing fiber Bragg gratings, the grating transmission spectrum is recorded with a Broadband Source (from JDSU) and an optical spectrum analyzer (OSA). A LabVIEW program has been compiled on a computer to control the OSA and to record the measured data. 


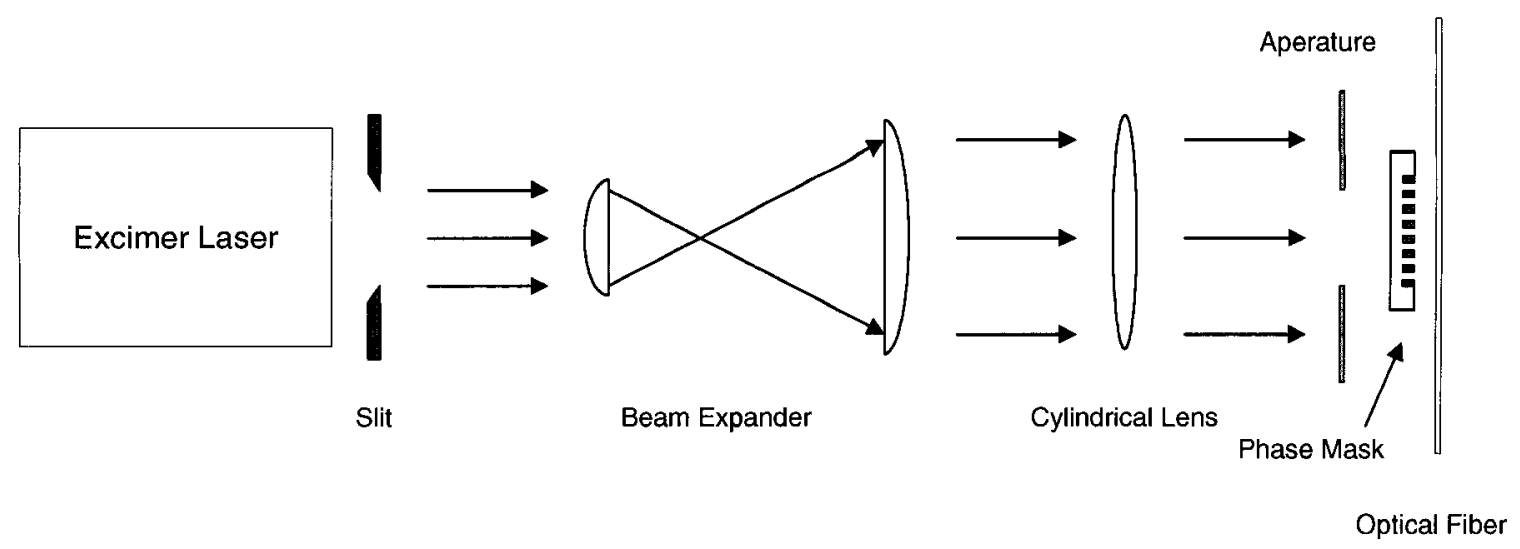

Fig 3.1 Schematic of a fiber Bragg grating fabrication system (Top view)

\subsubsection{Quick annealing technique and target erasure}

Once a Bragg grating is written on the hydrogen loaded fiber, it has to be subjected to a final annealing process before it is ready to use, since the hydrogen loaded FBGs demonstrates much lower thermal stability than nonhydrogenated FBGs [36]. This procedure, where typically the hydrogen loaded FBGs are annealed at $120^{\circ} \mathrm{C}$ for 12 hours, is aimed to stabilize the hydrogen loaded FBGs by removing the most unstable fraction of grating refractive index change. In addition, this process also forces the residual hydrogen molecules to leak out from the fiber because the diffusion rate of hydrogen is increased at a high temperature.

In contrast to a typical procedure to stabilize the hydrogen-loaded FBGs, our method incorporates an extra quick annealing step prior to the normal anneal process of the FBGs. The quick annealing step is carried out by using a heat gun to blow hot air on the FBG for a few minutes. This is a higher temperature process than the normal annealing, and could rapidly remove a large portion of the UV-induced refractive index change. For quick annealing, our target erasure portion is set to approximately $30 \%$ in refractive index modulation amplitude, which is nearly the maximum amount that can be erased in such a 
short time span. In order to keep all parts of the FBG annealed uniformly, one have to continuously sweep the heat gun back and forth between the FBG's ends. In addition, it must be ensured that the annealing temperature is below $300^{\circ} \mathrm{C}$, i.e. a safe temperature at which the thermal decay of grating does not involve any other complex mechanisms [16]. The limitation on the annealing temperature can be easily controlled by monitoring the Bragg wavelength. Note that the quick annealing with the heat gun is performed manually, and therefore the annealing temperature for each grating is randomly varies below $300{ }^{\circ} \mathrm{C}$ throughout the process. This varying annealing step may alter the defect energy state distribution of each grating in a different way, even though the amount of erasure of the grating strength is the same for all gratings. This can probably lead to different subsequent thermal decay behaviors of the gratings. This point will be discussed in more detail in future chapters.

Following a quick annealing, a normal annealing procedure is performed to heat the hydrogen loaded FBGs by baking the gratings in an oven (SH-241 from ESPEC) at $120^{\circ} \mathrm{C}$ for approximately 12 hours. After all these steps are completed, gratings are considered thermally stabilized and ready to be subjected to the accelerated aging experiment.

\subsection{Typical Results}

\subsubsection{Uniform fiber Bragg grating spectra (Transmission and Reflection)}

With the use of a BroadBand source (from JDSU), an optical spectrum analyzer (AQ6317B from Ando) and a $3 \mathrm{~dB}$ coupler, the transmission or reflection spectrum of a fiber Bragg grating can be measured. 


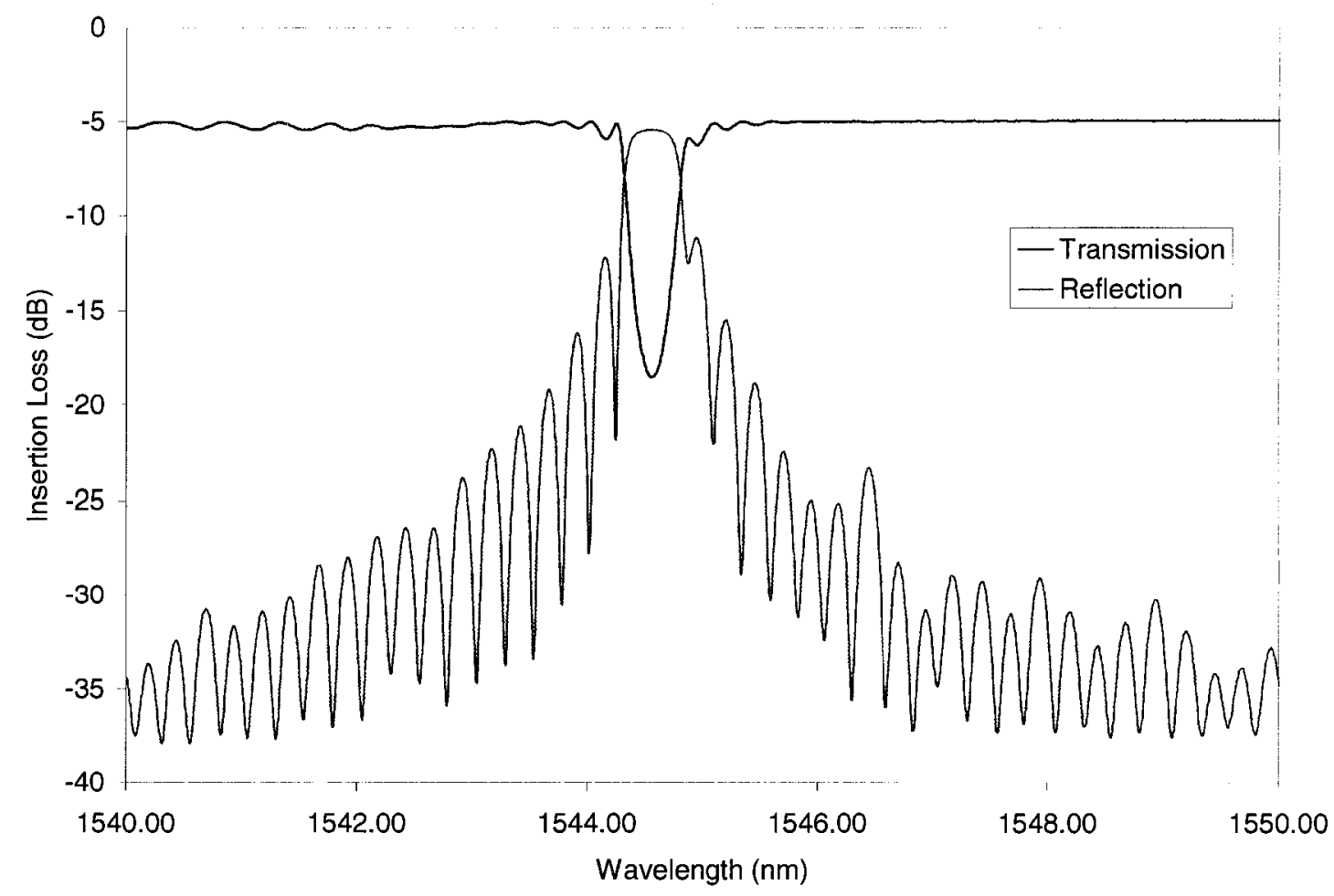

Fig 3.2 Transmission and reflection spectrum of a uniform fiber Bragg grating

A typical spectral response of a uniform FBG is shown in Fig 3.2, where the transmission and reflection of the grating are plotted with blue and pink solid lines respectively. Centering at the wavelength of $1544.550 \mathrm{~nm}$, the grating with a length of $3 \mathrm{~mm}$ has a notch depth of $13.53 \mathrm{~dB}$ in its transmission spectrum, which corresponds to a refractive index modulation of $4.88 \times 10^{-4}$. On the short wavelength side of the Bragg peak, there are several small cladding resonances whose attenuation depths are close to $0 \mathrm{~dB}$. These cladding resonances originate in the light coupling from the forward propagating fundamental mode to the backward propagating cladding modes. This is different from the Bragg reflection, which is related to light coupling between the counter propagating fundamental modes. As these cladding modes are of higher order than the fundamental mode and hence have smaller effective refractive index, the cladding resonance 
wavelengths of a grating are always smaller than its Bragg wavelength according to the Bragg condition. The fact that this sample grating is written without significant cladding resonances demonstrates that incidental laser beam is almost perfectly aligned normally to the fiber and no tilted grating is formed.

The reflection of the grating has a $3 \mathrm{~dB}$ bandwidth of $0.500 \mathrm{~nm}$, and a center wavelength of $1544.552 \mathrm{~nm}$, which agrees with its counterpart in the grating transmission spectrum. A pronounced feature of the grating reflection spectrum is the presence of side lobes. These side lobes appear symmetrically on both sides of the center peak, suggesting that a perfectly uniform Bragg grating has been fabricated in the fiber. Although a Gaussian apodized grating could suppress those side lobes, a grating with uniform structure of index change is more suitable for the study of thermal decay because it is easier to quantify, and can demonstrate the underlying mechanism more prominently.

\subsubsection{Grating growth}

As the Bragg wavelength and the transmission notch depth have been recorded consistently during the grating inscription, it is easy to follow the evolution of both the $\mathrm{DC}$ and the $\mathrm{AC}$ components of the UV induced refractive index change by using Eq. (2.11) and Eq. (2.13).

Fig 3.3 shows the grating growth behavior during the formation of hydrogen loaded fiber Bragg gratings. Both the mean refractive index change $\Delta n_{\text {mean }}$ and the refractive index modulation $\Delta n_{\text {mod }}$ for two gratings are illustrated as a function of exposure time. These two gratings are written by UV pulse irradiation at a pulse repetition rate of $40 \mathrm{~Hz}$ with 
fluence per pulse of $\sim 189 \mathrm{~mJ} / \mathrm{cm}^{2}$, whereas the only difference of their inscription parameters is the distance between the phase mask and the fiber: $600 \mu \mathrm{m}$ and $100 \mu \mathrm{m}$.

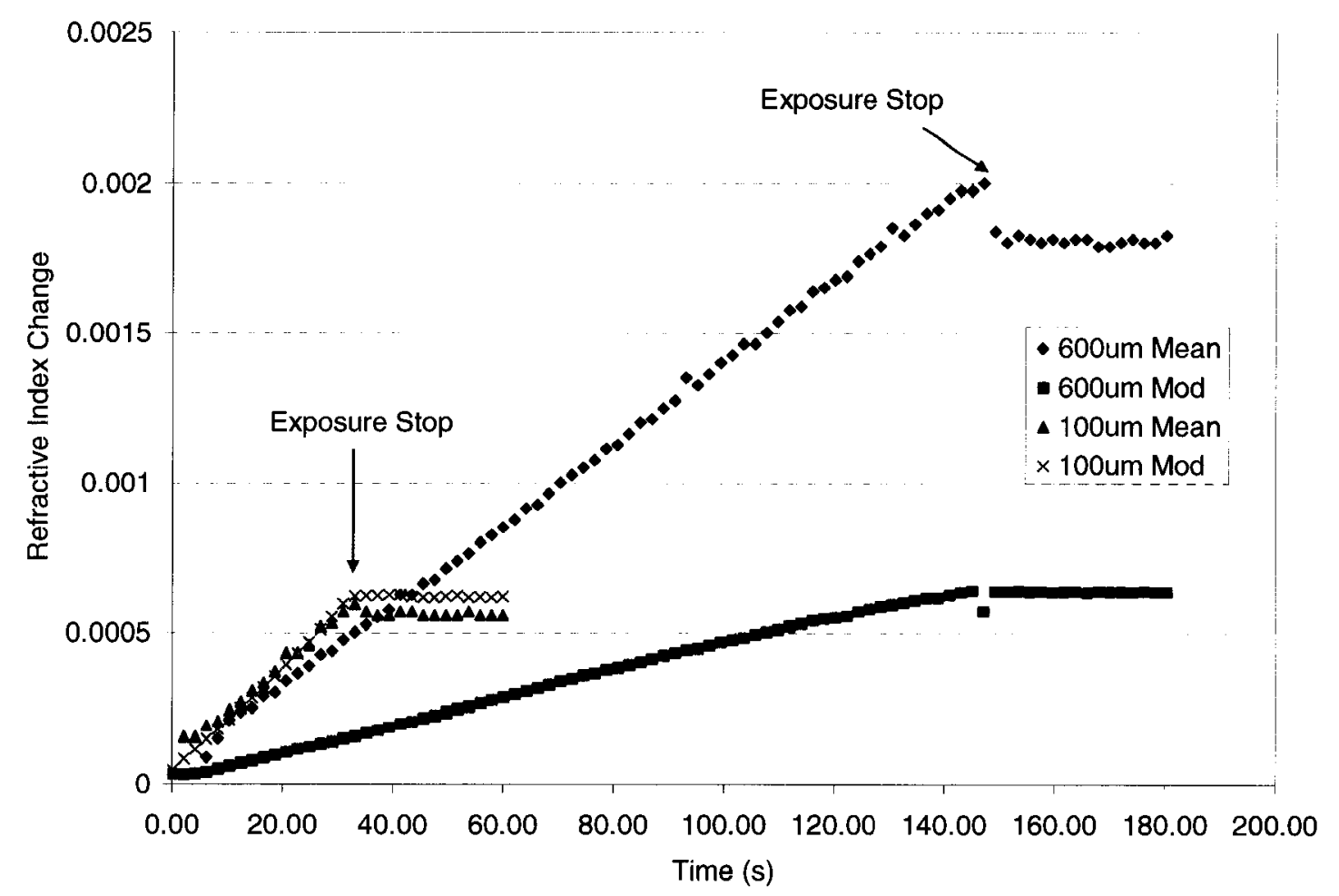

Fig 3.3 Grating growth of both the mean refractive index change and the refractive index modulation of gratings written at fiber/mask distance of $100 \mu \mathrm{m}$ and $600 \mu \mathrm{m}$

For the grating written at $600 \mu \mathrm{m}$, a refractive index modulation of $6.40 \times 10^{-4}$ was produced by an irradiation time of $147.03 \mathrm{~s}$ or a total incident fluence of $\sim 1.111 \mathrm{~kJ} / \mathrm{cm}^{2}$, and the average refractive index change was approximately $2.00 \times 10^{-3}$ before stopping exposure, which corresponds to a red shift of $1.60 \mathrm{~nm}$ of the Bragg wavelength during the irradiation.

In contrast, for the grating written at $100 \mu \mathrm{m}$, an irradiation time of $33.01 \mathrm{~s}$ or a total incident fluence of $\sim 0.249 \mathrm{~kJ} / \mathrm{cm}^{2}$ induced a similar refractive index modulation of $6.23 \times 10^{-4}$. However, the formed average refractive index change was only $5.96 \times 10^{-4}$ 
before stopping exposure, which corresponds to a red shift of $0.48 \mathrm{~nm}$ of the Bragg wavelength during the irradiation.

As illustrated in Fig 3.3, both the $\mathrm{DC}$ and the $\mathrm{AC}$ components of the refractive index change increase nearly linearly as a function of the exposure time. Furthermore, the growth rate of the refractive index modulation at the writing distance of $600 \mu \mathrm{m}$ is significantly lower than that at $100 \mu \mathrm{m}$, which supports the postulate that fringe contrast decreases with the increase of fiber/mask distance.

At the end of irradiation, the grating Bragg wavelength shifts towards the blue side of the spectrum, and this corresponds to the sudden decline of $\Delta n_{\text {mean }}$ from the linear growth to a steady level once the irradiation is stopped, as shown in the figure. In comparison, the index modulation keeps unchanged when the irradiation is stopped. This suggests that the blue shift of the Bragg wavelength originated in a decrease in the temperature of the fiber, which has been elevated by the UV pulse irradiation. This wavelength shift of the $600 \mu \mathrm{m}$ grating is $0.13 \mathrm{~nm}$, which indicates a temperature difference of roughly $13{ }^{\circ} \mathrm{C}$. In contrast, the wavelength shift of the other grating is only $0.03 \mathrm{~nm}$, and thus a temperature decrease of only $3{ }^{\circ} \mathrm{C}$. The reason for this strange difference lies in the absorption coefficient of the hydrogen loaded fiber at the irradiated UV wavelength region. It has been found that the absorption coefficient of a hydrogen loaded fiber grows when a 248 $\mathrm{nm}$ UV intense light is used to illuminate it [39]. As the grating written at $600 \mu \mathrm{m}$ was irradiated for longer time than the other, its improved absorption coefficient contributes to a larger amount of UV energy being absorbed, part of which was converted to heat. To show that the higher temperature difference is not simply due to accumulation of heat over the longer irradiation time, an additional experiment is carried out. As the irradiation 
is stopped and the wavelength blue shift occurs, an additional illumination of the grating with a few pulses causes the grating wavelength to instantly shift back to its original value, and it returns back to the blue shifted wavelength again once the UV irradiation is cut off.

\subsubsection{Grating visibility (AC/DC contrast ratio)}

Based on the grating growth curve, grating visibility (AC/DC contrast ratio of grating refractive index change) can be calculated by using experimental values after UV exposure stops, where the value of the mean refractive index change is more accurate.

Fig 3.4 illustrates the dependence of AC/DC contrast ratio of as-written hydrogen loaded FBGs on the fiber/phase mask distance. In order to improve measuring accuracy, 6 samples have been fabricated at each writing distance. The mean value of AC/DC contrast ratio at each writing distance is listed in Table 3.1. As shown in Fig 3.4, the grating index contrast decreases monotonically as the writing distance changes from 100 $\mu \mathrm{m}$ to $600 \mu \mathrm{m}$. Therefore, our goal of obtaining a larger DC component on gratings can be achieved by increasing the writing distance. This has been explained in Chapter 2 . Two interfering arms of the fringe pattern formed behind the phase mask lose correlation gradually as the separation between the fiber and the phase mask increases, and the interference fringe visibility becomes correspondingly lower. After $600 \mu \mathrm{m}$, if the writing distance keeps increasing, the AC/DC contrast ratio of grating will drop down gradually and eventually reach to zero, i.e. no refractive index modulation is formed on the fiber. This means that fiber/phase mask distance exceeds a critical value, at which the 
separation between the interfering arms on the phase mask is beyond the spatial coherence of the incident UV beam and a fringeless interfering field is formed.

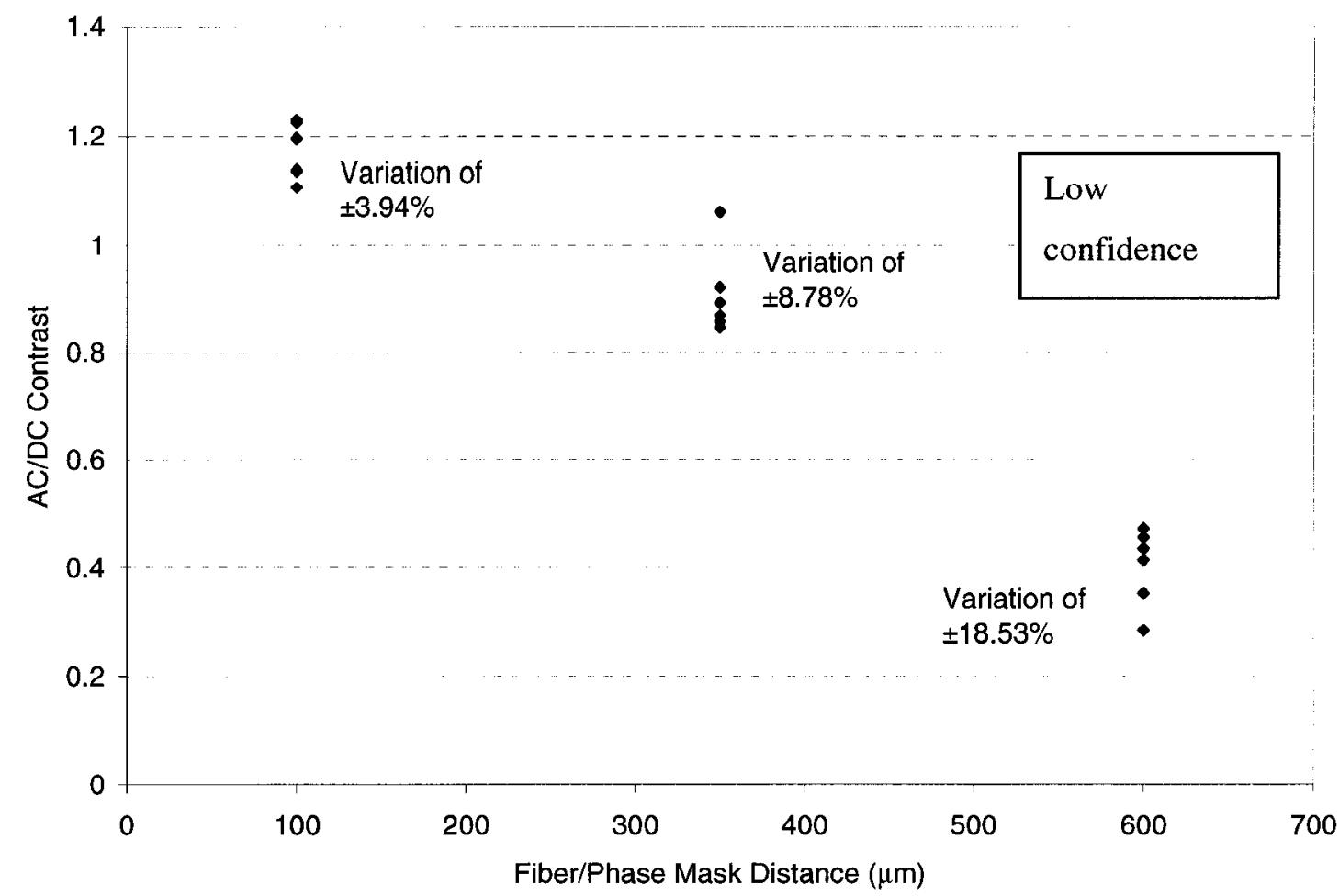

Fig 3.4 The dependence of grating index contrast (AC/DC) on the fiber/phase mask distance for hydrogen-loaded gratings.

\begin{tabular}{|c|c|}
\hline Fiber/Mask Distance $(\mu \mathrm{m})$ & Grating Index Contrast \\
\hline 100 & $1.195 \pm 3.94 \%$ \\
\hline 350 & $0.907 \pm 8.78 \%$ \\
\hline 600 & $0.402 \pm 18.53 \%$ \\
\hline
\end{tabular}

Table 3.1 Grating index contrast versus fiber/phase mask distance for gratings.

It is surprising that the $\mathrm{AC} / \mathrm{DC}$ index contrast appears to be larger than 1 at the distance of $100 \mu \mathrm{m}$, i.e. an AC component larger than a DC counterpart is induced. In fact, the DC 
index change calculated herein is an approximate value less than the real one, because the effect of hydrogen concentration on refractive index has been ignored. During the fabrication process, the hydrogen content decreases and this causes a decrease in the mean refractive index of the fiber [40-41]. The difference between the DC and the AC index change should therefore account for this refractive index contribution. Although we have low confidence in these calculated DC components, they can still provide a good indication on the trend of the AC/DC contrast ratio as writing distance varies.

Another pronounced feature of the grating fabrication is the variation of the AC/DC contrast ratio at each writing distance, which is indicated in the figure, especially at the largest distance. Even though all samples at each distance were fabricated consecutively in order to diminish any variations in the writing conditions, each grating still shows a different index contrast ratio. Furthermore, the variation of the grating index contrast is larger at a longer writing distance than at a shorter distance.

To demonstrate the origin of this variation clearly, grating growths of all gratings fabricated at $600 \mu \mathrm{m}$ are plotted together in Fig 3.5 for comparison. It can be seen that there is a slight difference in the growth rate of the refractive index modulation between the different gratings. Therefore, the exposing times used for the fabrication differed greatly with each fiber, and longer inscription times caused a higher mean refractive index change to be reached for the same final value of index modulation. This finally leads to a great difference on the mean refractive index change formed on the fiber.

This variation of the grating index contrast may be due to a number of varying grating writing conditions, such as fluctuation of the pulse energy, the hydrogen concentration of 
the optical fibers, and displacement of fiber from the focus of the laser beam. All these effects are worse when larger fiber/phase mask distances are used.

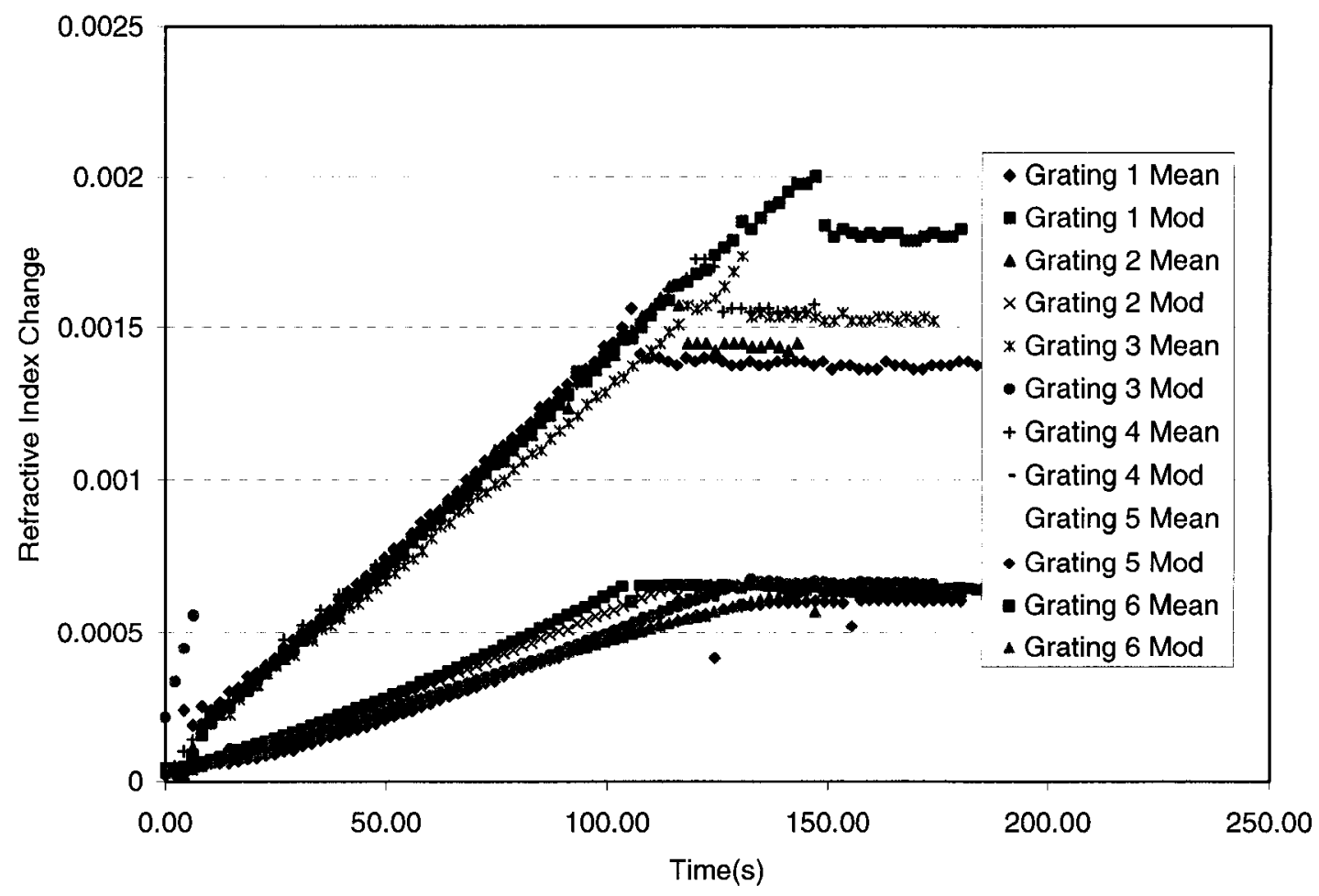

Fig 3.5 Grating growths of refractive index change for 6 gratings written at $600 \mu \mathrm{m}$.

\subsubsection{Variation of grating period}

One interesting observation of our fabrication experiment is a small variation of the actual value of grating period formed in the fibers. For each writing distance, we formed a pristine grating on nonhydrogenated SMF-28 through only a few pulses exposure. As the UV induced refractive index change on the grating is negligible, the Bragg wavelength of this grating (which can only be observed in its small reflection peak) should always be the same for any writing distance, equal to twice of product of the effective index of pristine SMF-28 and the grating period according to the Bragg condition. However, several experimental results demonstrated that the Bragg 
wavelengths of pristine gratings either increase or decrease as the writing distance increases. As the effective index of each optical fiber has no reason to change upon exposure of a few pulses, the deviation of the Bragg wavelength (around $0.1 \mathrm{~nm}$ ) only results from the variation of grating period, which could only be attributed to the variation of interference fringe pattern due to the Gaussian behavior of the incident laser beam. As a converging beam propagates through the phase mask, the period of interfering fringe pattern will decrease along the fiber/phase mask distance. In contrast, a diverging incident beam leads to an increase on the grating period at a long writing distance. This has been proven by adjusting the beam expander before the phase mask, and hence altering the beam behavior into either a diverging or a converging beam. In our experiment, the laser beam is always expected to be adjusted as a parallel beam, so the relative deviation of grating period is only about $0.01 \%$, which is negligible enough to assume the grating period is fixed and is equal to half that the period of the phase mask. However, this effect can probably find an application in determining the beam divergence angle of a laser with high accuracy.

\subsection{Accelerated Aging Set-up}

\subsubsection{Customized micro-furnace design}

Once the hydrogen-loaded FBGs have been fabricated and pre-annealed, they are ready to be subjected to different thermal decay experiments. The experimental setup for performing thermal decay (or accelerated aging) on multiple fiber Bragg gratings simultaneously will be introduced here. 

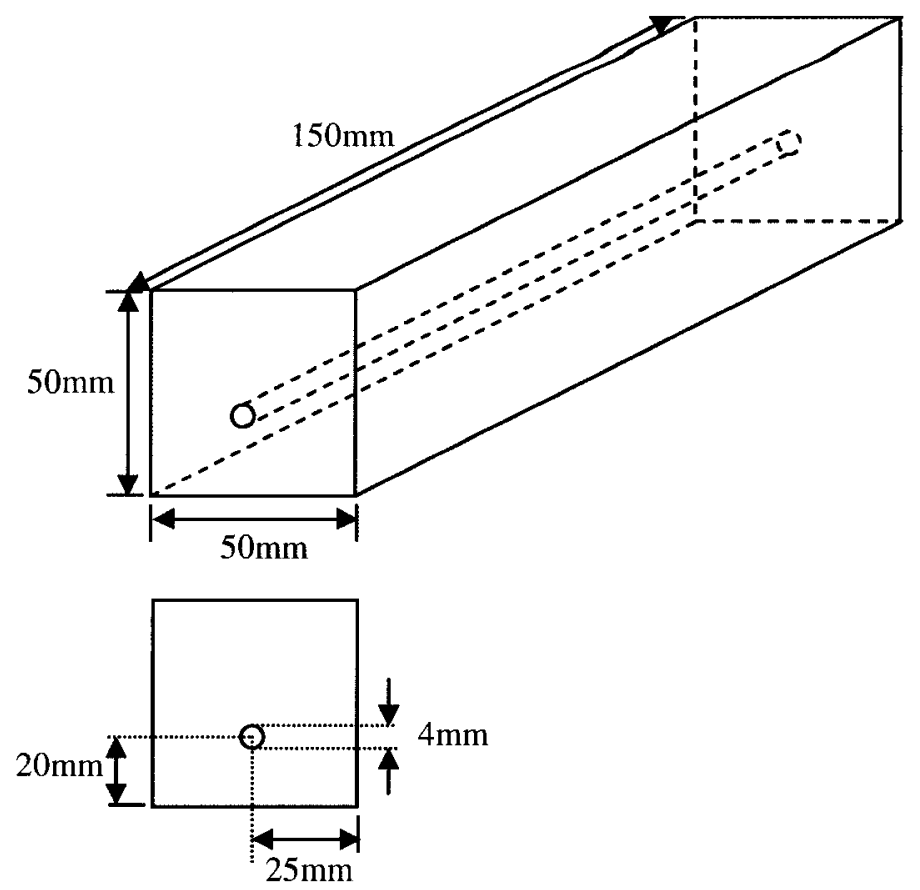

Fig 3.6 The design diagram of a rectangular tube employed as micro furnace.

Since we did not have a good oven working at high temperature, a self-customized micro furnace was designed for our accelerated aging experiment of FBGs. As illustrated in Fig 3.6 , this micro furnace is a rectangular block with a small tunnel made along its long direction, inside which fiber Bragg gratings are placed. This micro furnace can be heated to a high temperature by mounting it on a flat heating plate. The material of the micro furnace is selected as copper owing to its excellent thermal conductivity, and its dimensions are illustrated in the figure. The diameter of the tunnel is only $4 \mathrm{~mm}$ in order to keep heating space compact and minimize the influence of temperature fluctuation from free air flow. The tunnel length of $15 \mathrm{~cm}$, which is much longer than the typical grating length of $1 \mathrm{~cm}$, ensures that the temperature in the grating area is uniform and steady. 
As the tunnel is filled many fibers, it becomes difficult to measure the temperature inside the tube by insertion of a thermocouple. Therefore, in order to monitor the temperature inside the tube, a hole with a small diameter of $1 \mathrm{~mm}$ is perpendicularly drilled from the front side, ending at the center of the bulk which is in close proximity of the grating position. Repeated experiments have demonstrated that the temperature inside the tiny hole is only about $1.0{ }^{\circ} \mathrm{C}$ less than that inside the furnace tunnel in the operating temperature range of $200{ }^{\circ} \mathrm{C}$ to $300{ }^{\circ} \mathrm{C}$. With this small temperature difference calibration, the temperature at the tiny hole can be used as a reliable and accurate reference for the operating temperature of the micro furnace.

\subsubsection{Accelerated aging set-up}

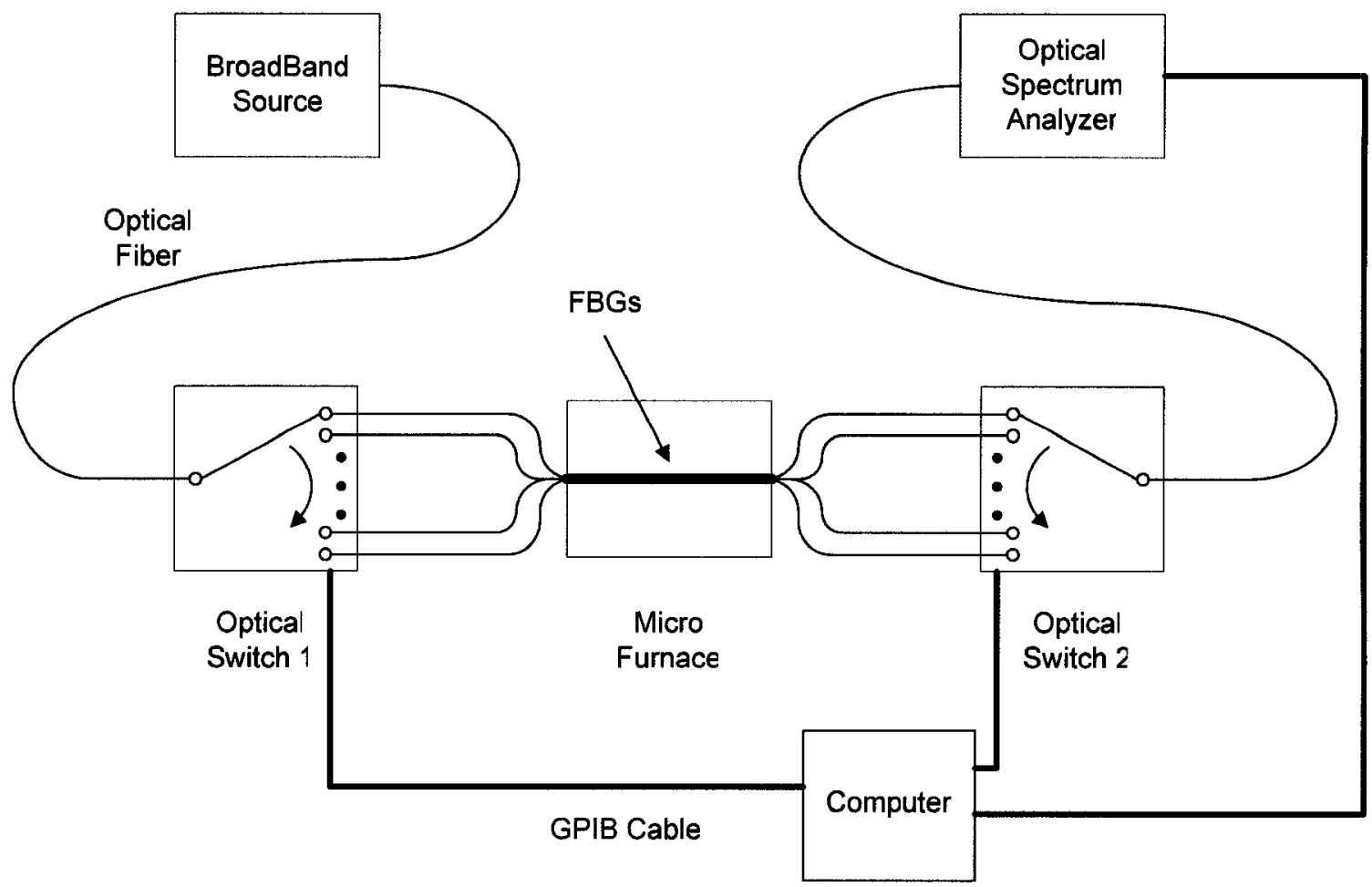

Fig 3.7 Schematic of the accelerated aging experimental setup for the measurement of multiple gratings 
Fig 3.7 depicts the schematic arrangement of the accelerated aging experiment setup, which enables simultaneous measurement of multiple grating transmission spectra as a function of annealing time. It consists of a broadband source, a pair of $1 \times 19$ optical switches, a self-customized micro furnace, FBGs under detection, and an optical spectrum analyzer. Light emitted from the broadband source (Erbium-doped fiber amplified spontaneous emission source from JDSU) propagates through a section of optical fiber, and launches into the input port of the optical switch 1 (SB series from JDSU). On the other side of this optical switch, FBGs under detection are sandwiched between this switch and its counterpart optical switch 2 , in such a way that each grating is connected to two corresponding output ports of the optical switches. The output port of optical switch 2 is connected to an optical spectrum analyzer (AQ6317B from Ando), which is used to measure the transmission spectra of the FBGs. The wavelength resolution of the OSA is set at $0.01 \mathrm{~nm}$.

For thermal annealing, all FBGs are bundled together and placed inside the small tunnel of our self-customized micro furnace, which has reached the desired annealing temperature after a few hours of warm up. During annealing, the temperature of the micro furnace is monitored by using a K-type thermocouple, placed inside the monitoring side hole of the micro furnace. The resolution of the thermometer (HH506RA from Omega) is $0.1{ }^{\circ} \mathrm{C}$. By checking and controlling the temperature periodically during the test, the temperature variation is maintained in the range of $\pm 5{ }^{\circ} \mathrm{C}$ due to the fluctuation of ambient temperature. In this annealing experiment setup, the optical switches and the OSA are connected to a computer through GPIB (General Purpose Interface Bus) cables. 
A Labview program is configured to automatically control the equipment and record data for multiple gratings over a long period of time.

Optical switches are used to switch between all the desired channels consecutively and repeatedly. Once the OSA accumulates the data for one channel, the measurement of next channel is carried out immediately and thus the interval between two adjacent channels is the single sweeping time of the OSA, which depends on its measurement parameters setup, such as the wavelength resolution, the average times, and the sweeping wavelength span. In our experiment, the interval is approximately 8 seconds after a compromise has been reached between the accuracy of the measurements and the measurement interval of the channels.

\subsubsection{Problems and solutions for data processing method}

As a parameter to measure the grating decay, the reflectivity of the grating could be determined by the maximum attenuation depth in the transmission spectrum. The attenuation was measured as the difference (in $\mathrm{dB}$ ) of the transmission minimum relative to a flat reference adjacent to the Bragg wavelength. The wavelength of the flat reference should be selected far enough from the Bragg wavelength to avoid the effect of grating spectrum on this reference power. For instance, the reference wavelength should be at least $2 \mathrm{~nm}$ more than Bragg wavelength in Fig 3.2.

In addition, to avoid the effects of power fluctuation of the broadband source, which may cause additional error in the reflectivity of the gratings, measurements at two fixed wavelength (start and stop wavelengths of the OSA sweeping range) are also recorded as additional references. Experimental results demonstrate that the power fluctuation of the 
broadband source is less than $0.16 \mathrm{~dB}$ over a period time of several weeks. This error can be neglected for thermal decay experiment on gratings with attenuation depth of more than $10 \mathrm{~dB}$. Moreover, this small power variation will only affect measured grating reflectivity partly or even not at all, since grating reflectivity is a relative measurement on power level instead of absolute one. Despite the small influence of power fluctuation, data at start and stop sweeping wavelength are still recorded accompanying with data at attenuation peak and flat reference. This three-reference points method apparently makes experimental acquisition data more reliable.

As mentioned in the last chapter, grating refractive index modulation is typically normalized to 1 with its initial value prior to annealing for the following analysis on the grating thermal decay pattern. In order to obtain a reliable initial value of reflectivity in our case, a measurement is done at room temperature before putting the grating in the heated micro furnace. However, using initial refractive index modulation at room temperature directly as normalization factor was found to be not accurate, and a slight correction should be made on this value. Thermal annealing experiment results demonstrate that in additional to an irreversible change, i.e. thermal decay, the reflectivity of Bragg gratings also experiences a slight reversible change as temperature of grating increases from room temperature to a higher temperature. In terms of refractive index modulation, it will decrease approximately $1 \%$ for a rise of the grating temperature from room temperature to $200^{\circ} \mathrm{C}$, and will return to its initial value once temperature reduces to room temperature. Fig 3.8 shows this reversible change of index modulation $\left(\Delta n_{\bmod }(T) / \Delta n_{\bmod }\left(23^{\circ} C\right)\right)$ for our hydrogen-loaded FBGs at various high temperatures. 


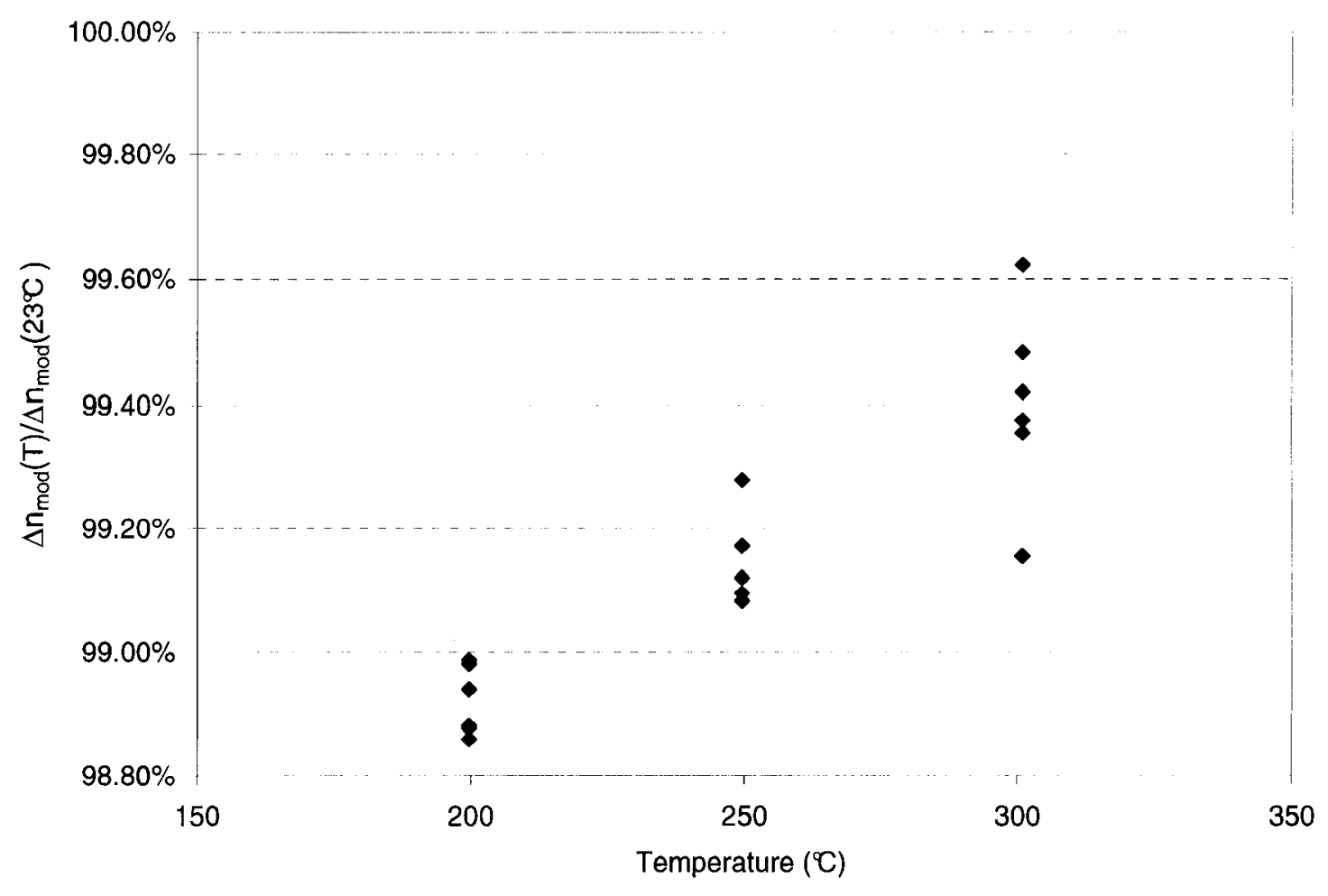

Fig 3.8 Normalized change in the grating index modulation as a function of temperature $\mathrm{T}\left({ }^{\circ} \mathrm{C}\right)$

The observed temperature induced reversible change in refractive index modulation has been suggested to originate in the structural change during the formation of the FBG that alter some thermal property of the glass in correlation with a change in the density of the glass [42]. In more detail, it has been proposed that the thermal expansion coefficient has been altered locally, and a modulation of the thermal expansion coefficient has been produced during the exposure to the fringe pattern. An increase in the fiber temperature will induce different stresses at the bright and dark regions of the grating, thus changing the refractive index modulation of the grating.

Comparing with the above paper, our experimental results demonstrate that hydrogen loaded FBGs do exhibit this kind of temperature induced reversible change effect, which 
was reported to not exist in hydrogen loaded FBGs. This may be due to a different mechanism involved in our case.

Although the observed temperature induced reversible change in refractive index modulation is very small, a correction to the normalization factor of refractive index modulation is still carried out for the sake of accuracy. Therefore, we take the initial refractive index modulation at the annealing temperature, to be equal to the initial refractive index modulation measured at room temperature multiplied by a correction factor, the reversible change $\Delta n_{\text {mod }}(T) / \Delta n_{\bmod }\left(23^{\circ} C\right)$. 


\section{Chapter 4}

\section{Experimental results}

Accelerated aging tests employing an isothermal annealing method will be carried out on two groups of pre-annealed gratings, which have different grating index modulation amplitudes. Both groups of gratings are samples fabricated at various fiber/phase mask distances. Detailed information about these samples is given in the first part of this chapter. Following that, the thermal decay curves of these hydrogen loaded pre-annealed gratings are given, and the fitting results, which utilize the simple power decay model, are also presented.

\subsection{Description of the sample sets}

In order to fabricate the FBGs for the thermal decay experiment, standard telecommunication single mode fibers (Corning SMF-28) were loaded with hydrogen at room temperature for 14 days at $\sim 17.24 \mathrm{Mpa}$. Phase mask technology was employed for the inscription of FBGs on these hydrogen-loaded fibers by the use of UV light from a pulsed $\mathrm{KrF}$ excimer laser at $248 \mathrm{~nm}$. Two groups of uniform type I FBGs have been written within hydrogen-loaded single mode fibers: strong gratings and weak gratings in terms of the grating's index modulation amplitude.

Strong gratings with $3 \mathrm{~mm}$ grating length, all of which have approximately same aswritten refractive index modulation amplitude $\Delta n_{\text {mod }}$ of $\sim 6.60 \times 10^{-4}\left(6.22 \times 10^{-4}\right.$ to $6.91 \times 10^{-4}$ ) corresponding to $\sim 99.07 \%(98.65 \%$ to $99.29 \%)$ in reflectivity, were fabricated 
at different fiber/phase mask distance $(100 \mu \mathrm{m}, 350 \mu \mathrm{m}$ and $600 \mu \mathrm{m})$ in order to introduce different mean refractive index changes $\Delta n_{\text {mean }}$. Similarly, refractive index modulation amplitude $\Delta n_{\text {mod }}$ of $\sim 2.57 \times 10^{-4}$ was introduced on hydrogen-loaded fibers with grating length of $10 \mathrm{~mm}$, and these weak gratings with reflectivity of $\sim 99.92 \%$ also had attained different mean refractive index changes by following the same inscribing method as before.

To this end, 18 strong gratings were formed, of which every 6 gratings were fabricated using the same displacement between the phase mask and the fiber, and had nearly the same DC and AC refractive index changes. As for the weak gratings, we fabricated 36 gratings in total: 6 specimens of each individual displacement of $50 \mu \mathrm{m}, 100 \mu \mathrm{m}, 150$ $\mu \mathrm{m}, 200 \mu \mathrm{m}, 3$ specimens of each displacement of $250 \mu \mathrm{m}, 300 \mu \mathrm{m}, 350 \mu \mathrm{m}, 2$ samples for $600 \mu \mathrm{m}$, and 1 sample for $800 \mu \mathrm{m}$. Detailed information about all the fabricated gratings is shown in the Table 4.1 and Table 4.2. Before starting the isothermal annealing test, all the hydrogen-loaded gratings were pre-annealed after fabrication to remove most of the unstable portion of the grating's UV-induced index change. About $30 \%$ of the grating's index modulation was erased during this process, which is widely considered to be adequate for the stabilization of hydrogen-loaded gratings. However, due to loose control on the quick-annealing procedure as mentioned in Chapter 3, the erased portion of index modulation varies for each grating as shown in both tables below. 


\begin{tabular}{|r|l|l|l|l|l|}
\hline $\begin{array}{l}\text { Distance } \\
(\mu \mathrm{m})\end{array}$ & $\begin{array}{l}\text { As-written } \\
\text { Refractive } \\
\text { index } \\
\text { modulation } \\
\Delta n_{\text {mod }}\end{array}$ & $\begin{array}{l}\text { As-written } \\
\text { Mean } \\
\text { refractive } \\
\text { index change } \\
\Delta n_{\text {mean }}\end{array}$ & $\begin{array}{l}\text { Pre-annealed } \\
\text { Refractive } \\
\text { index } \\
\text { modulation } \\
\Delta n_{\text {mod }}\end{array}$ & $\begin{array}{l}\text { Pre-annealed } \\
\text { Mean } \\
\text { refractive } \\
\text { index change } \\
\Delta n_{\text {mean }}\end{array}$ & $\begin{array}{l}\text { Pre-annealed } \\
\text { portion of } \\
\text { index } \\
\text { modulation } \\
\Delta n_{\text {mod }}\end{array}$ \\
\hline 100 & 0.000657 & 0.000558 & 0.000477 & 0.000515 & $27.41 \%$ \\
\hline 100 & 0.000668 & 0.000553 & 0.000482 & 0.000515 & $27.79 \%$ \\
\hline 100 & 0.000667 & 0.000575 & 0.000467 & 0.000503 & $30.09 \%$ \\
\hline 100 & 0.000692 & 0.00057 & 0.000484 & 0.000515 & $30.02 \%$ \\
\hline 100 & 0.000673 & 0.000547 & 0.000485 & 0.000528 & $27.93 \%$ \\
\hline 100 & 0.000622 & 0.00056 & 0.000478 & 0.000553 & $23.25 \%$ \\
\hline 350 & 0.000676 & 0.000747 & 0.000483 & 0.000782 & $28.58 \%$ \\
\hline 350 & 0.000647 & 0.000707 & 0.000486 & 0.000744 & $24.99 \%$ \\
\hline 350 & 0.000665 & 0.000783 & 0.000487 & 0.000794 & $26.72 \%$ \\
\hline 350 & 0.00066 & 0.000773 & 0.000485 & 0.000782 & $26.58 \%$ \\
\hline 350 & 0.000654 & 0.000751 & 0.000483 & 0.000794 & $26.11 \%$ \\
\hline 350 & 0.000655 & 0.000751 & 0.000473 & 0.000744 & $27.73 \%$ \\
\hline 600 & 0.000646 & 0.001387 & 0.000481 & 0.001395 & $25.43 \%$ \\
\hline 600 & 0.000651 & 0.001436 & 0.000483 & 0.001507 & $25.81 \%$ \\
\hline 600 & 0.000662 & 0.001547 & 0.000488 & 0.001557 & $26.27 \%$ \\
\hline 600 & 0.000639 & 0.001563 & 0.000471 & 0.001645 & $26.26 \%$ \\
\hline 600 & 0.000636 & 0.001762 & 0.000481 & 0.001607 & $24.42 \%$ \\
\hline 600 & 0.000636 & 0.001802 & 0.000476 & 0.001882 & $25.15 \%$ \\
\hline
\end{tabular}

* Low confidence on values of $\Delta n_{\text {mean }}$

Table 4.1 Characteristics of index change for strong gratings 


\begin{tabular}{|c|c|c|c|c|c|}
\hline $\begin{array}{l}\text { Distance } \\
(\mu \mathrm{m})\end{array}$ & $\begin{array}{l}\text { As-written } \\
\text { Refractive } \\
\text { index } \\
\text { modulation } \\
\Delta n_{\text {mod }}\end{array}$ & $\begin{array}{l}\text { As-written } \\
\text { Mean } \\
\text { refractive } \\
\text { index change } \\
\Delta n_{\text {mean }}\end{array}$ & $\begin{array}{l}\text { Pre-annealed } \\
\text { Refractive } \\
\text { index } \\
\text { modulation } \\
\Delta n_{\text {mod }}\end{array}$ & $\begin{array}{l}\text { Pre-annealed } \\
\text { Mean } \\
\text { refractive } \\
\text { index change } \\
\Delta n_{\text {mean }}\end{array}$ & $\begin{array}{l}\text { Pre-annealed } \\
\text { portion of } \\
\text { index } \\
\text { modulation } \\
\Delta n_{\text {mod }}\end{array}$ \\
\hline 50 & 0.000264 & 0.000289 & 0.000186 & 0.000179 & $29.57 \%$ \\
\hline 50 & 0.000267 & 0.000289 & 0.000183 & 0.000168 & $31.44 \%$ \\
\hline 50 & 0.000254 & 0.000266 & 0.00018 & 0.000197 & $28.85 \%$ \\
\hline 50 & 0.000266 & 0.000272 & 0.00018 & 0.000167 & $32.19 \%$ \\
\hline 50 & 0.000264 & 0.000261 & 0.000183 & 0.000167 & $30.77 \%$ \\
\hline 50 & 0.000262 & 0.000275 & 0.000181 & 0.00023 & $30.77 \%$ \\
\hline 100 & 0.000255 & 0.000256 & 0.000179 & 0.000242 & $29.65 \%$ \\
\hline 100 & 0.000263 & 0.000261 & 0.000184 & 0.000192 & $29.97 \%$ \\
\hline 100 & 0.000257 & 0.000257 & 0.000185 & 0.000192 & $27.77 \%$ \\
\hline 100 & 0.000266 & 0.000261 & 0.000179 & 0.000167 & $32.85 \%$ \\
\hline 100 & 0.000262 & 0.000234 & 0.000183 & 0.000167 & $30.15 \%$ \\
\hline 100 & 0.000267 & 0.000256 & 0.000187 & 0.00018 & $29.81 \%$ \\
\hline 150 & 0.000263 & 0.000297 & 0.000188 & 0.000325 & $28.55 \%$ \\
\hline 150 & 0.00026 & 0.000284 & 0.000184 & 0.000312 & $29.32 \%$ \\
\hline 150 & 0.000258 & 0.000271 & 0.000183 & 0.000287 & $29.28 \%$ \\
\hline 150 & 0.000262 & 0.000282 & 0.00019 & 0.000287 & $27.53 \%$ \\
\hline 150 & 0.000264 & 0.000307 & 0.000186 & 0.000312 & $29.49 \%$ \\
\hline 150 & 0.00026 & 0.00027 & 0.000181 & 0.000275 & $30.67 \%$ \\
\hline 200 & 0.000261 & 0.000311 & 0.000185 & 0.000303 & $29.31 \%$ \\
\hline 200 & 0.00026 & 0.000349 & 0.000184 & 0.00034 & $29.15 \%$ \\
\hline 200 & 0.00026 & 0.000362 & 0.000184 & 0.000365 & $29.31 \%$ \\
\hline 200 & 0.000268 & 0.000309 & 0.000189 & 0.000315 & $29.51 \%$ \\
\hline 200 & 0.000268 & 0.000341 & 0.000185 & 0.000278 & $31.06 \%$ \\
\hline 200 & 0.000262 & 0.000385 & 0.000189 & 0.000365 & $28.08 \%$ \\
\hline 250 & 0.000265 & 0.000355 & 0.000188 & 0.00034 & $28.90 \%$ \\
\hline 250 & 0.000263 & 0.000322 & 0.000186 & 0.000302 & $29.03 \%$ \\
\hline 250 & 0.000262 & 0.000335 & 0.000181 & 0.000315 & $30.89 \%$ \\
\hline 300 & 0.000264 & 0.000371 & 0.000186 & 0.000365 & $29.70 \%$ \\
\hline 300 & 0.000256 & 0.000366 & 0.000179 & 0.000365 & $30.17 \%$ \\
\hline 300 & 0.000263 & 0.000354 & 0.000185 & 0.000352 & $29.81 \%$ \\
\hline 350 & 0.000265 & 0.000406 & 0.000187 & 0.000374 & $29.39 \%$ \\
\hline 350 & 0.000259 & 0.000419 & 0.000178 & 0.000354 & $31.07 \%$ \\
\hline 350 & 0.00026 & 0.000386 & 0.000183 & 0.00035 & $29.67 \%$ \\
\hline 600 & 0.000258 & 0.000475 & 0.000181 & 0.000489 & $29.75 \%$ \\
\hline 600 & 0.000266 & 0.000435 & 0.000184 & 0.000452 & $30.75 \%$ \\
\hline 800 & 0.000264 & 0.000851 & 0.000187 & 0.000742 & $29.35 \%$ \\
\hline
\end{tabular}

* Low confidence on values of $\Delta n_{\text {mean }}$

Table 4.2 Characteristics of index change for weak gratings 


\subsection{Accelerated Aging results}

Accelerated aging tests are carried out on both groups of gratings by using the setup shown in Fig 3.7. Here, the isothermal annealing method is employed for the aging test. In an isothermal annealing, the grating is exposed to a fixed temperature and the degradation of its strength is monitored as a function of time. More specifically, the isothermal annealing processes for both groups of gratings were performed at fixed temperatures of $200^{\circ} \mathrm{C}, 250{ }^{\circ} \mathrm{C}$, and $300^{\circ} \mathrm{C}$.

\subsubsection{Thermal decay of strong hydrogen-loaded FBGs}

For strong gratings, all 18 gratings were divided into 3 groups, each group made up of three pairs of gratings (one pair for each index contrast) and each group being subjected to a different isothermal annealing process. By using two nominally identical gratings for each situation (index contrast and temperature) we hope to obtain a rough estimate of the experimental error that would impact any model-based lifetime predictions. The experimental data were accumulated by measuring the transmission spectra of all the gratings every 60 seconds, and gratings were held at their annealing temperature $\left(200^{\circ} \mathrm{C}\right.$, $250{ }^{\circ} \mathrm{C}$, and $300{ }^{\circ} \mathrm{C}$ ) for durations that were sufficient long (typically several weeks) to yield observable clear thermal decay characteristics. The large data sets obtained from these intensive observations contribute to a precise description of thermal decay and help in comparing the applicability of several fitting models. The results of the annealing experiments are summarized in Fig. 4.1 to Fig 4.6. In each case, the integrated coupling constant is normalized to its initial value before starting the aging experiment. It is called the normalized integrated coupling constant (NICC, same as normalized index 
modulation), and is used as the parameter representing thermal decay of grating as suggested by Erdogan [15].

Two special points about the way that we present the thermal decay results should be addressed. Take the case of thermal decay at $300^{\circ} \mathrm{C}$ as an example. First, the evolution of NICC as a function of the annealing time is plotted using both linear and log scale time axes in Fig 4.1(a) and (b), respectively. The logarithmic time axis plot is used in order to clearly show the decay at the beginning of the anneal process. Second, in addition to NICC normalized to the initial value prior to the isothermal annealing as in Fig 4.1, another type of NICC, which are the same annealing data normalized to the as-written value of ICC, are also plotted against annealing time in Fig 4.2. This procedure originates from the possibility that the pre-annealing of a $\mathrm{H}_{2}$-loaded $\mathrm{FBG}$ before isothermal annealing may change its subsequent decay pattern due to the historic dependence of thermal decay. With normalization to the as-written index modulation, thermal degradation of this NICC treats the pre-annealing and isothermal annealing as a whole thermal process.

When the thermal decay is plotted with linear vertical and horizontal (time) scales as in Fig. 4.1(a), each grating exhibits an initial sharp decrease in the NICC, followed by a progressively slower decay but with a nonzero rate of change. This feature of the thermal decay is fundamental to the practice of stabilizing grating structures by accelerated aging, which is generally carried out by heating the gratings at high temperature to force them to reach the slow decay region. In contrast, when thermal degradations of gratings is presented using a logarithmic time scale, each grating begins with an irregular decay pattern varying at different annealing temperatures, but converges nicely to a straight line 
for very long annealing times. As shown in these figures, the rate of change of the NICC as a function of the logarithm of time is almost zero at the beginning, especially in the case of isothermal decay at $200^{\circ} \mathrm{C}$, then increases with longer annealing time, and finally reaches an approximately linear decay rate for very long times. This characteristic of thermal decay indicates that thermal degradation of hydrogen-loaded, unsaturated, AND PRE-ANNEALED (removal of $30 \%$ of the index modulation prior to starting aging) gratings still comprises at least two different regimes with different characteristics for a short-term decay and a long-term decay. The latter is the only basis on which one can predict the grating's lifetime, and it appears to follow a very regular function law. Since the final slope of the long-term decay has a slight deviation from that of a straight line, the fitting model describing the long-term decay that we selected is a simple power function $N I C C=a t^{b}$ (see Eq. (2.26)) instead of the more common logarithm function $N I C C=1-k \cdot \ln (t / \tau)$ (see Eq. (2.24)).

In the remainder of this subsection, all the aging curves for strong gratings are presented, in the various formats included for completeness. For weak gratings, similar to strong gratings, all gratings were divided into 3 groups and subjected to isothermal annealing processes. The thermal decay curves of weak gratings are summarized in subsection 4.2.2 (Fig 4.7 to Fig 4.12).

The results will be analyzed and discussed in Chapter 5 . 


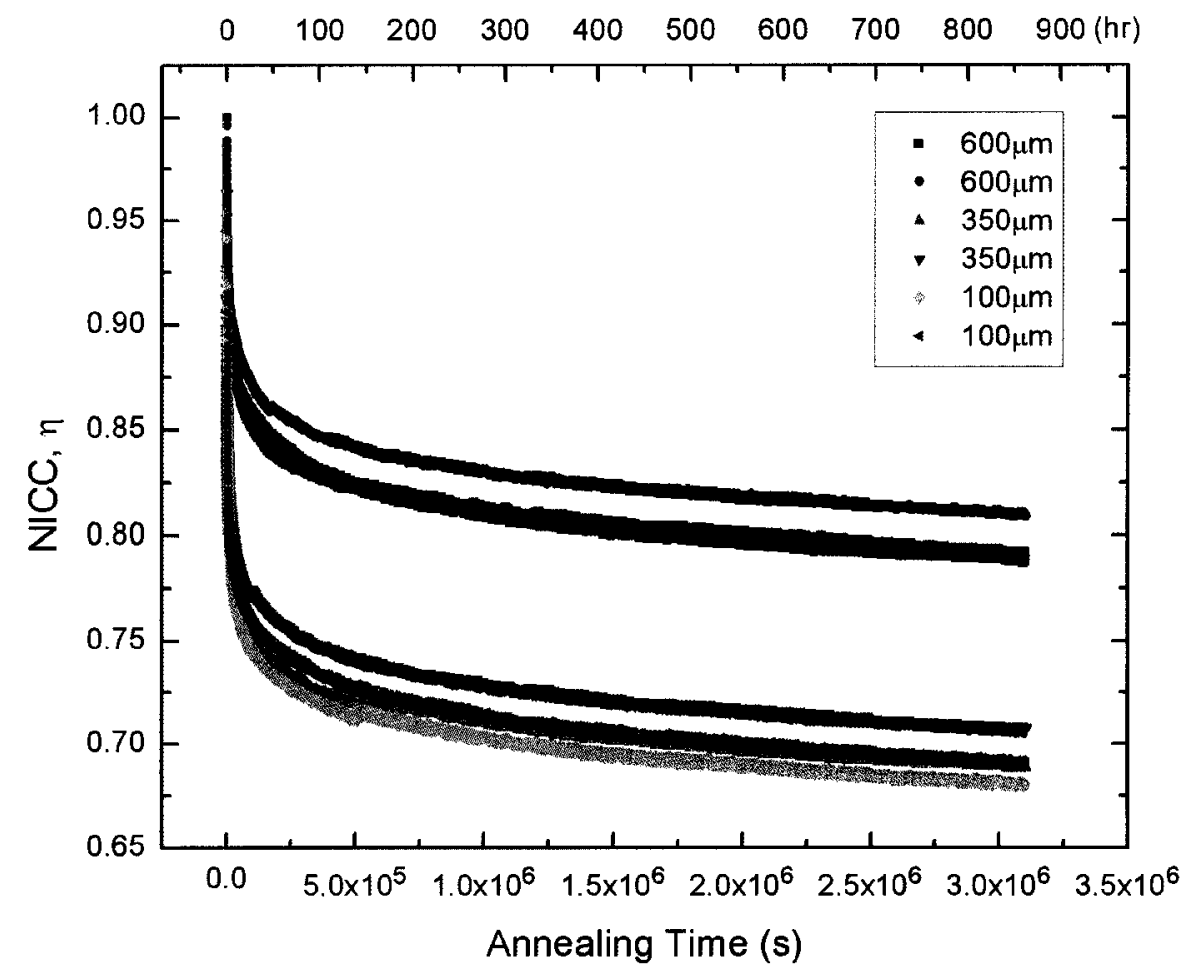

(a)

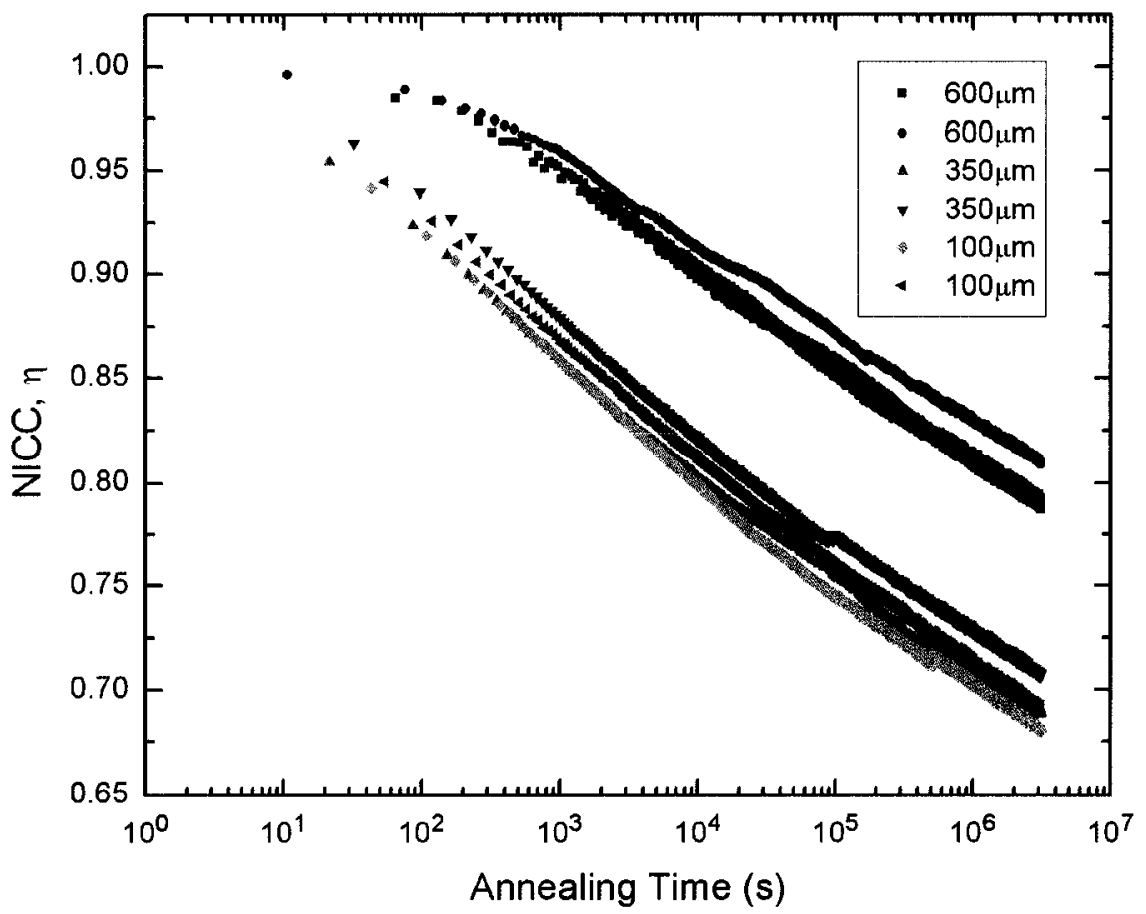

(b)

Fig 4.1 Isothermal decay of normalized integrated coupling constant (i.e. normalized refractive index modulation amplitude, normalized to the initial value at annealing temperature) for strong gratings as a function of annealing time at temperature of $300^{\circ} \mathrm{C}$ (a) linear time scale; (b) logarithmic time scale. 


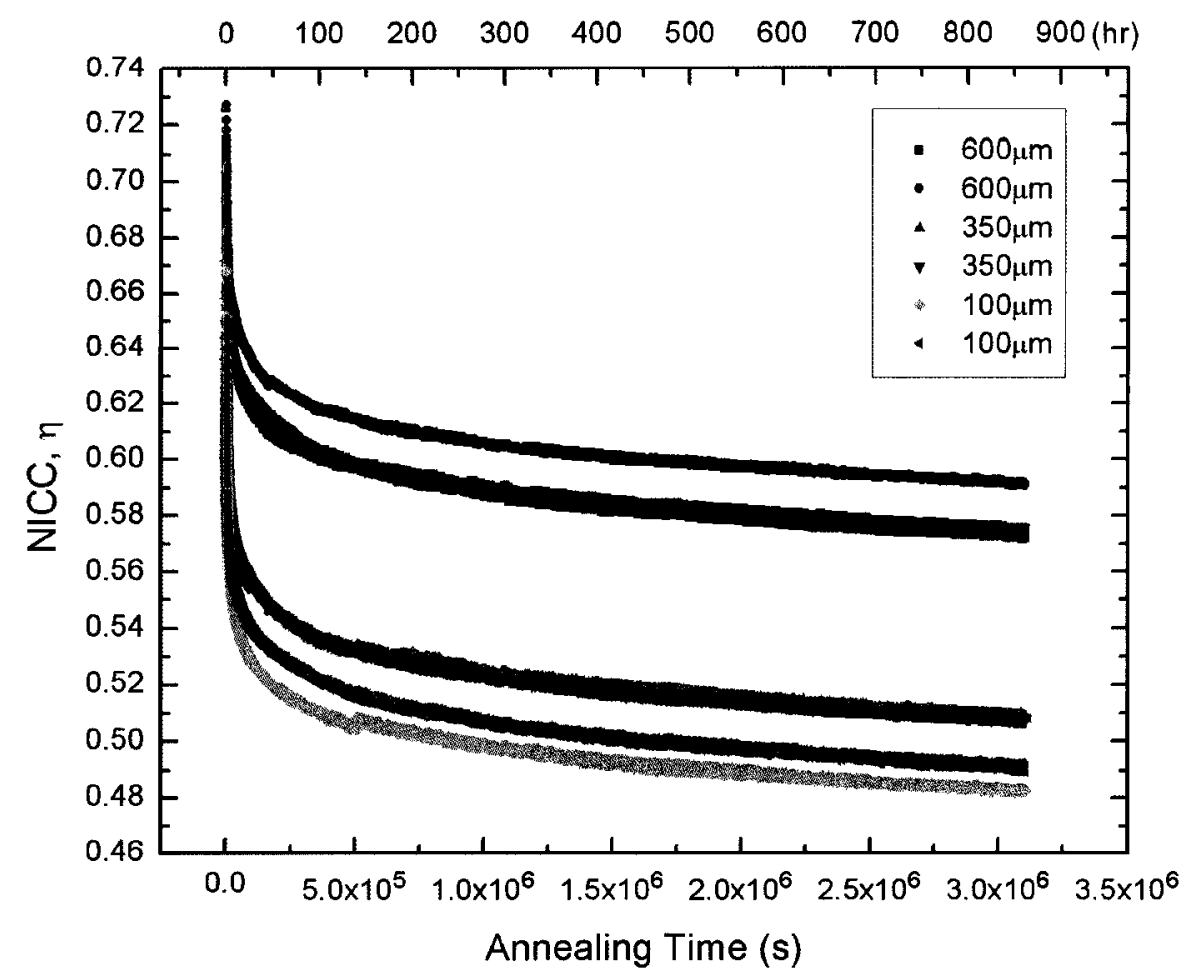

(a)

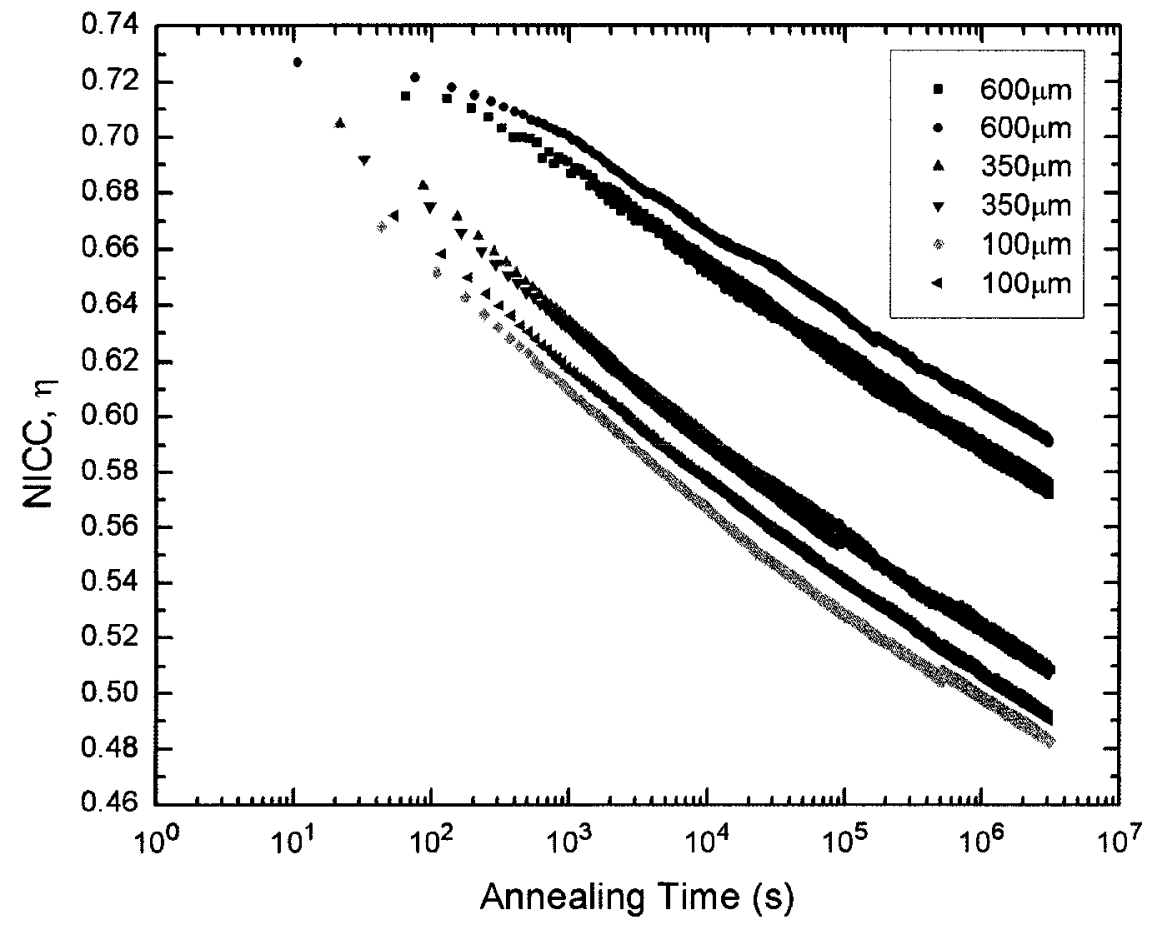

(b)

Fig 4.2 Isothermal decay of normalized integrated coupling constant (i.e. normalized refractive index modulation amplitude, normalized to the initial as-written value) for strong gratings as a function of annealing time at temperature of $300^{\circ} \mathrm{C}$ (a) linear time scale; (b) logarithmic time scale. 


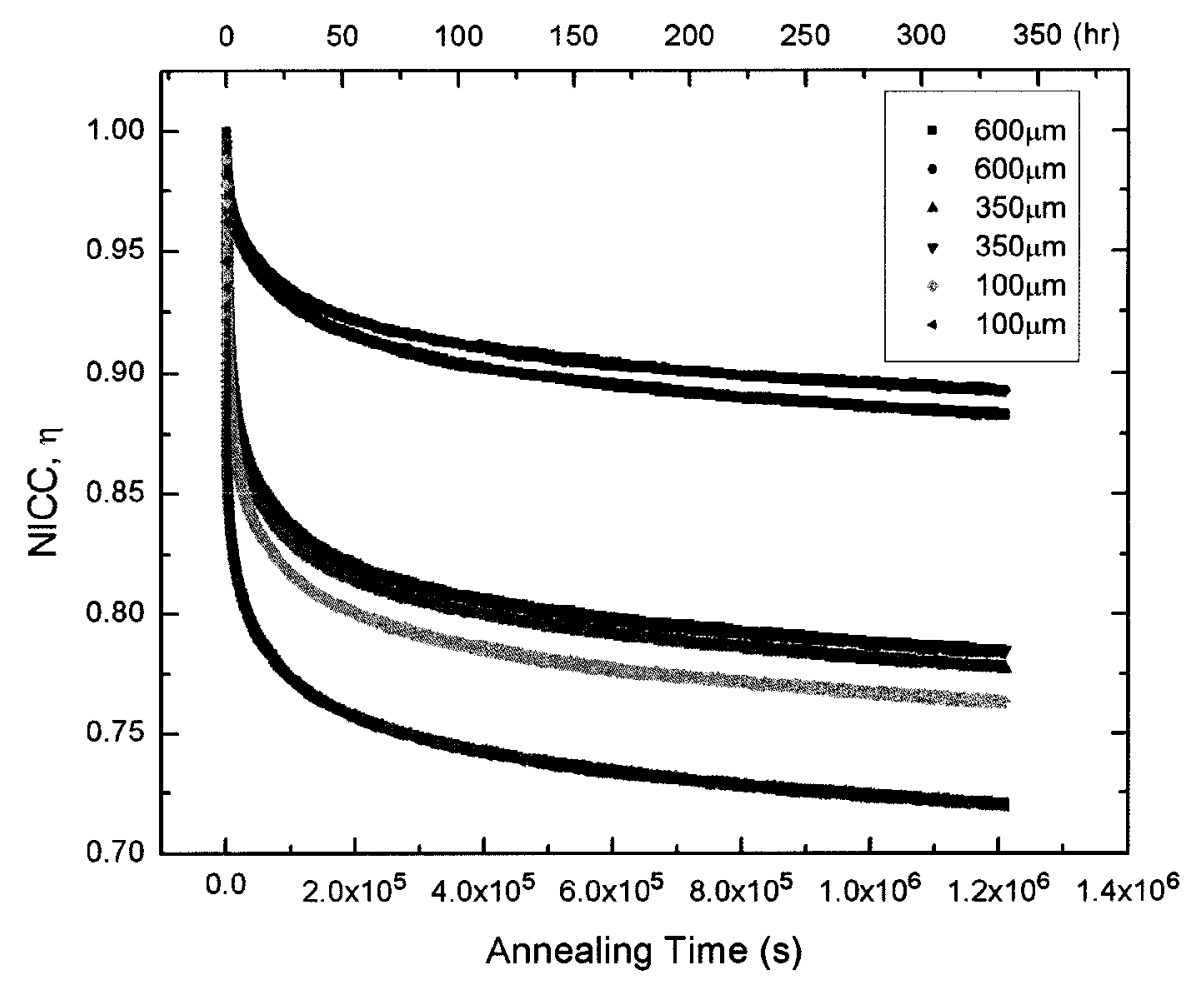

(a)

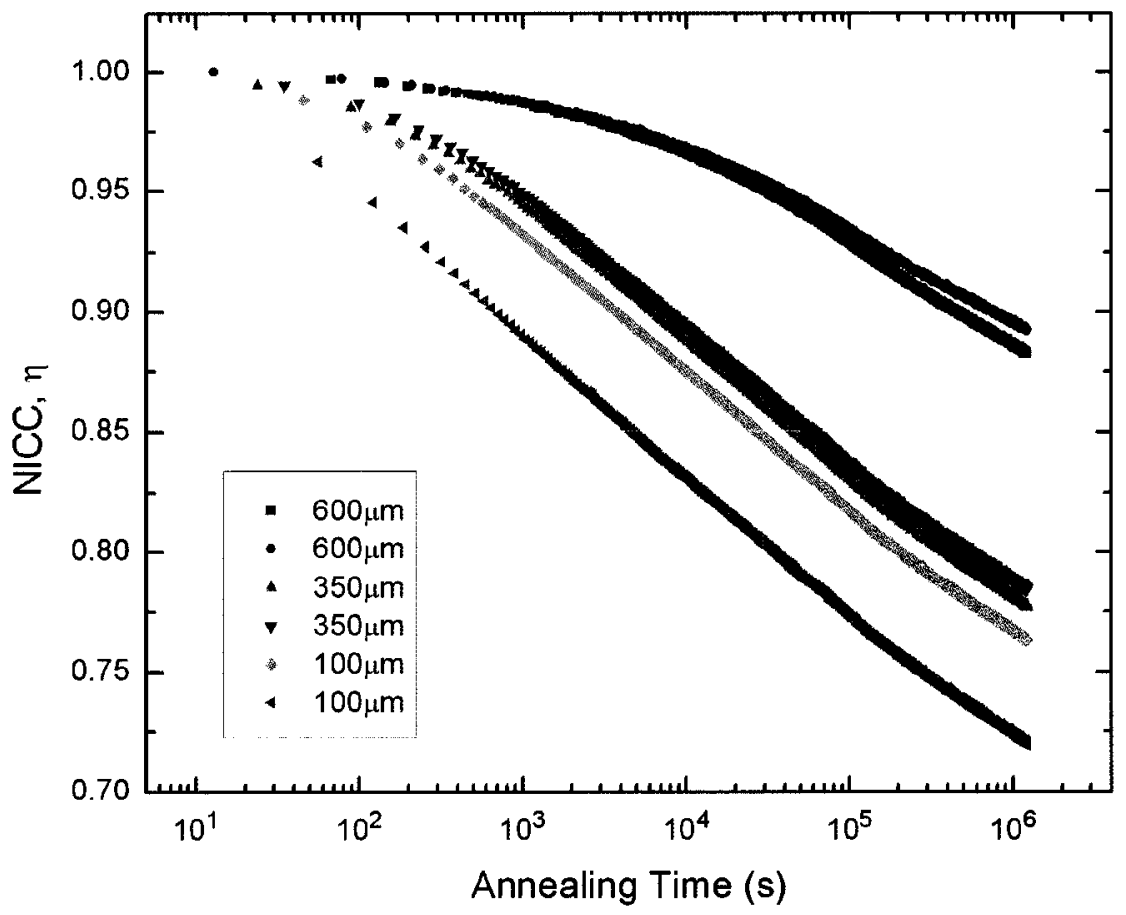

(b)

Fig 4.3 Isothermal decay of normalized integrated coupling constant (i.e. normalized refractive index modulation amplitude, normalized to the initial value at annealing temperature) for strong gratings as a function of annealing time at temperature of $250^{\circ} \mathrm{C}$ (a) linear time scale; (b) logarithmic time scale. 


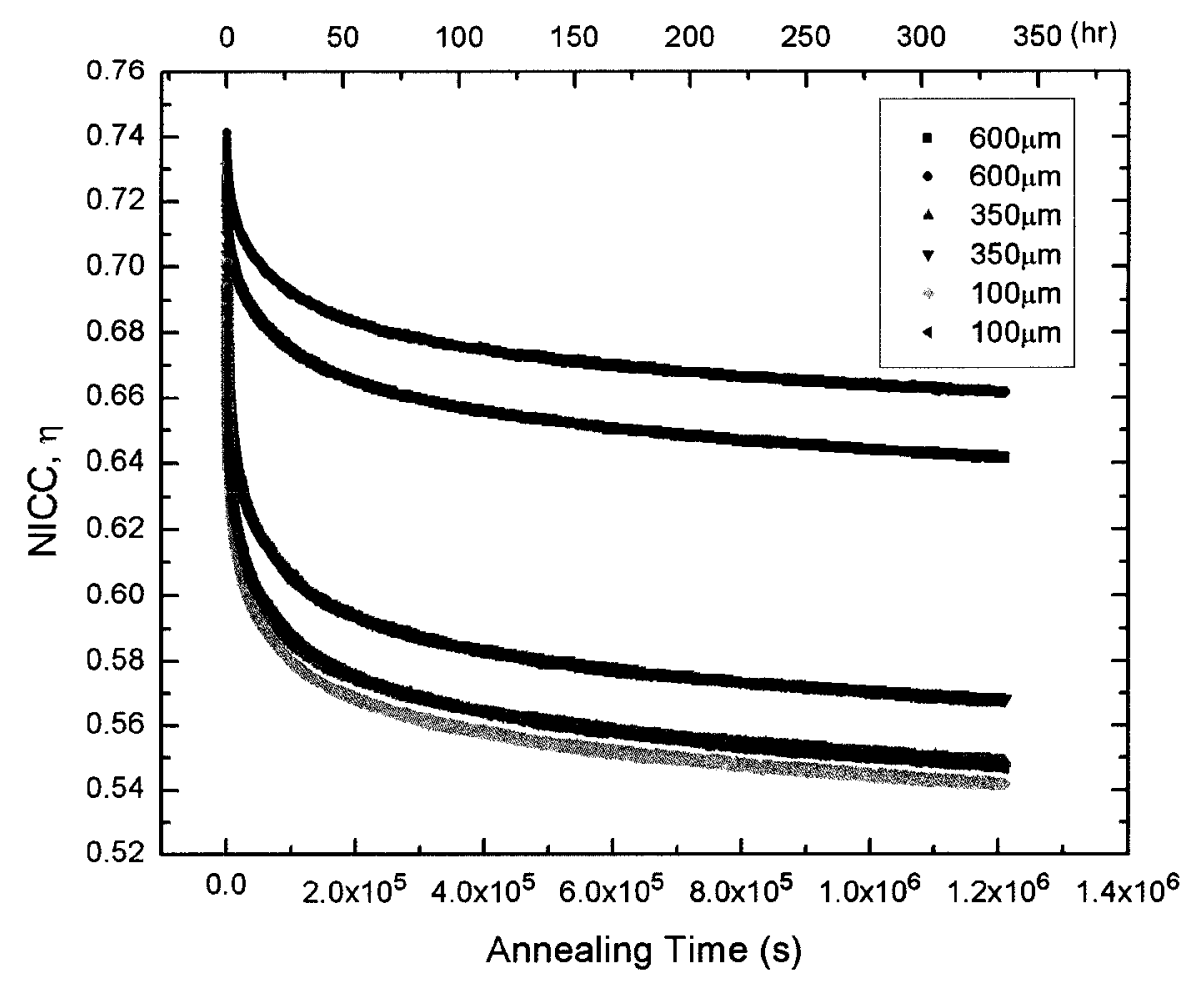

(a)

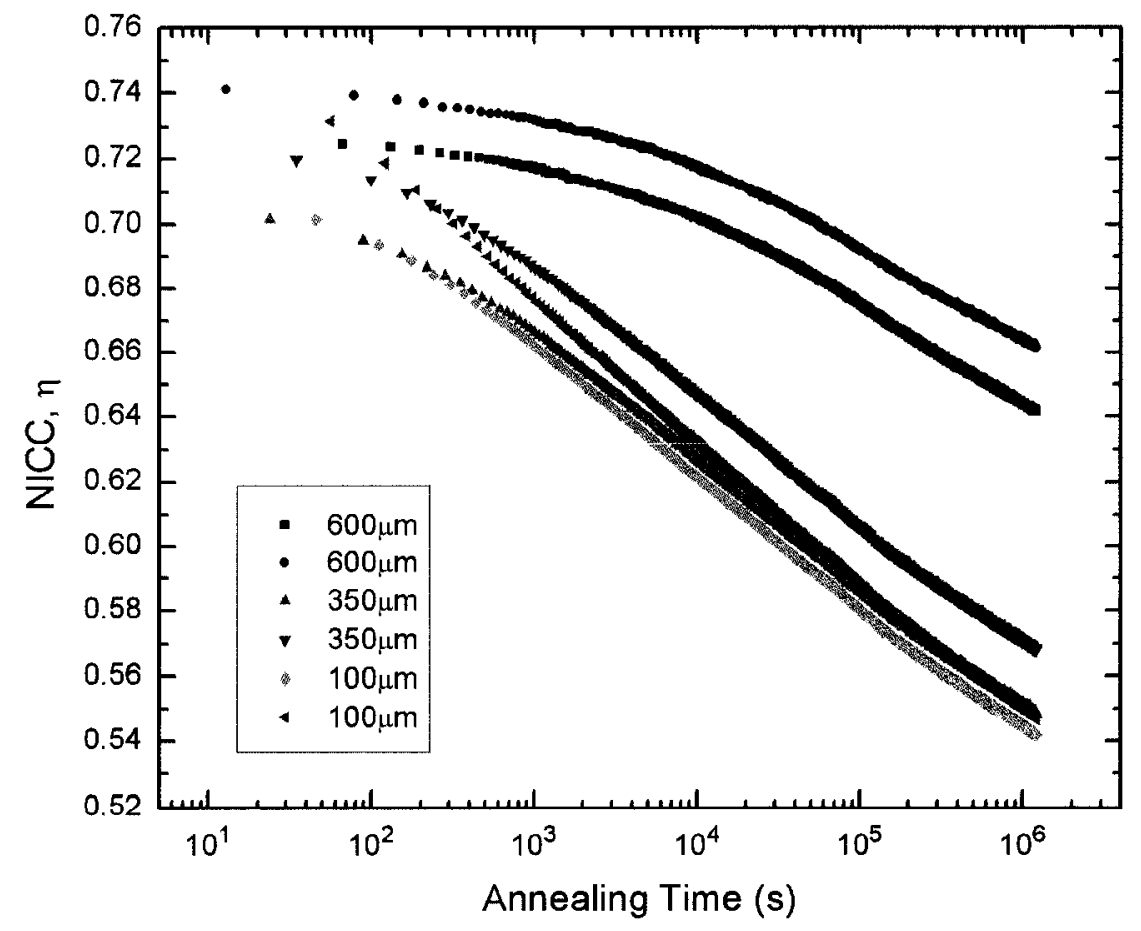

(b)

Fig 4.4 Isothermal decay of normalized integrated coupling constant (i.e. normalized refractive index modulation amplitude, normalized to the initial as-written value) for strong gratings as a function of annealing time at temperature of $250^{\circ} \mathrm{C}$ (a) linear time scale; (b) logarithmic time scale. 


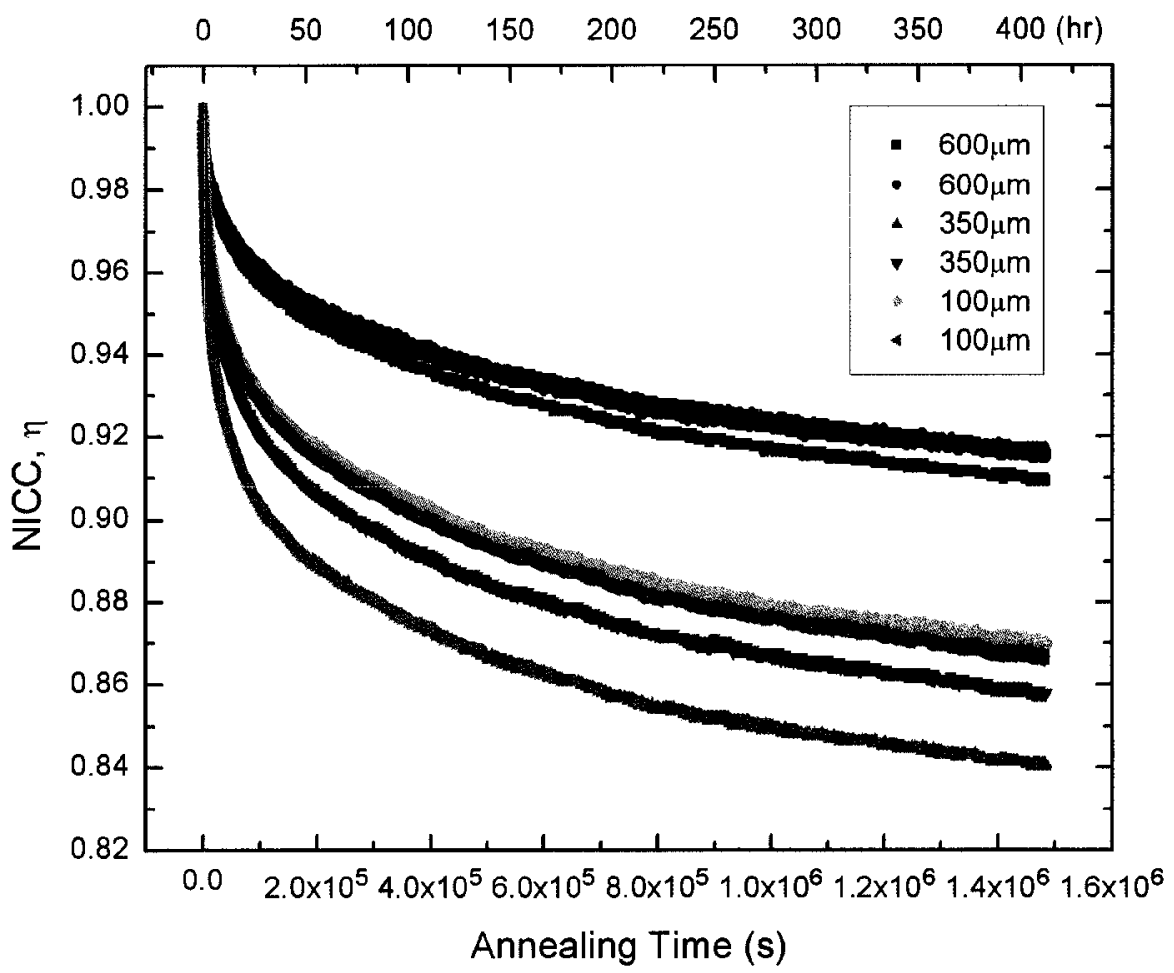

(a)

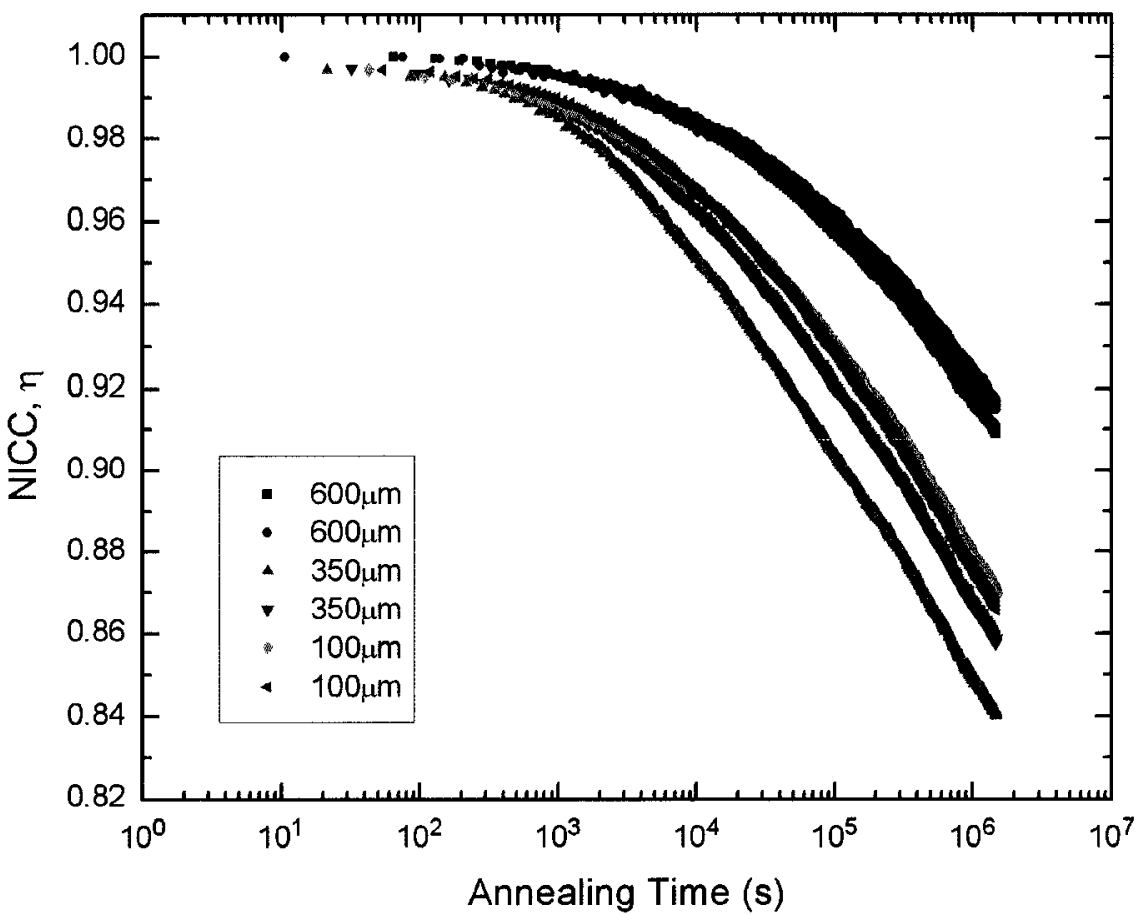

(b)

Fig 4.5 Isothermal decay of normalized integrated coupling constant (i.e. normalized refractive index modulation amplitude, normalized to the initial value at annealing temperature) for strong gratings as a function of annealing time at temperature of $200^{\circ} \mathrm{C}$ (a) linear time scale; (b) logarithmic time scale. 


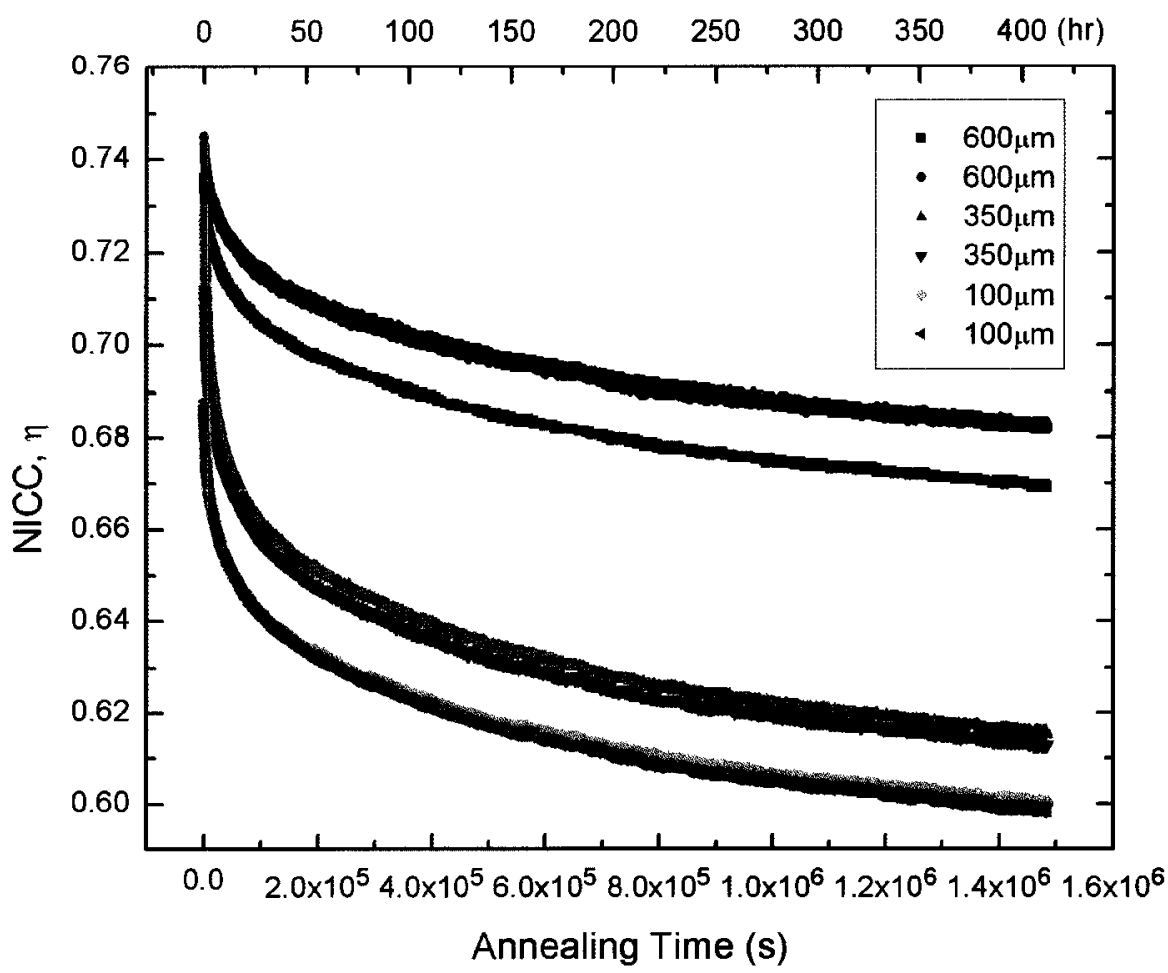

(a)

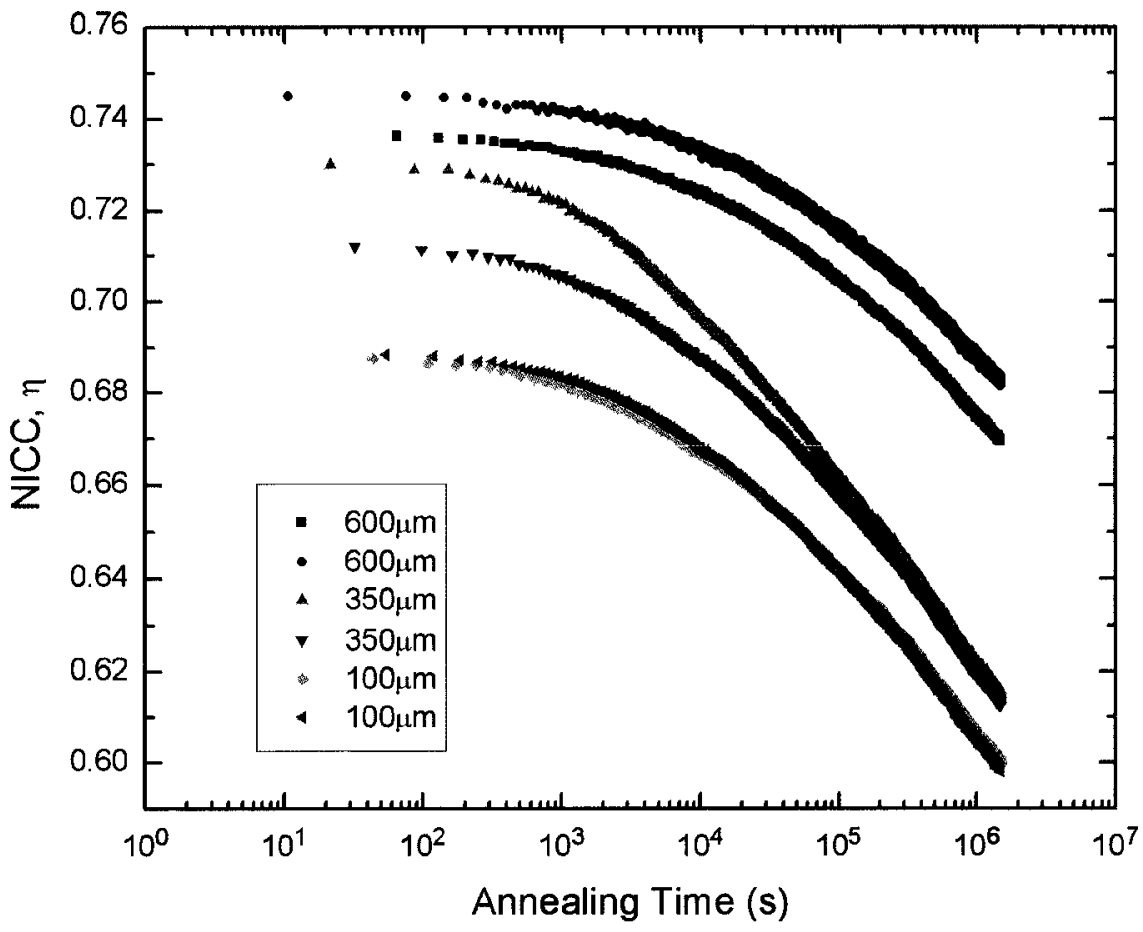

(b)

Fig 4.6 Isothermal decay of normalized integrated coupling constant (i.e. normalized refractive index modulation amplitude, normalized to the initial as-written value) for strong gratings as a function of annealing time at temperature of $200^{\circ} \mathrm{C}$ (a) linear time scale; (b) logarithmic time scale. 


\subsubsection{Thermal decay of weak hydrogen-loaded FBGs}

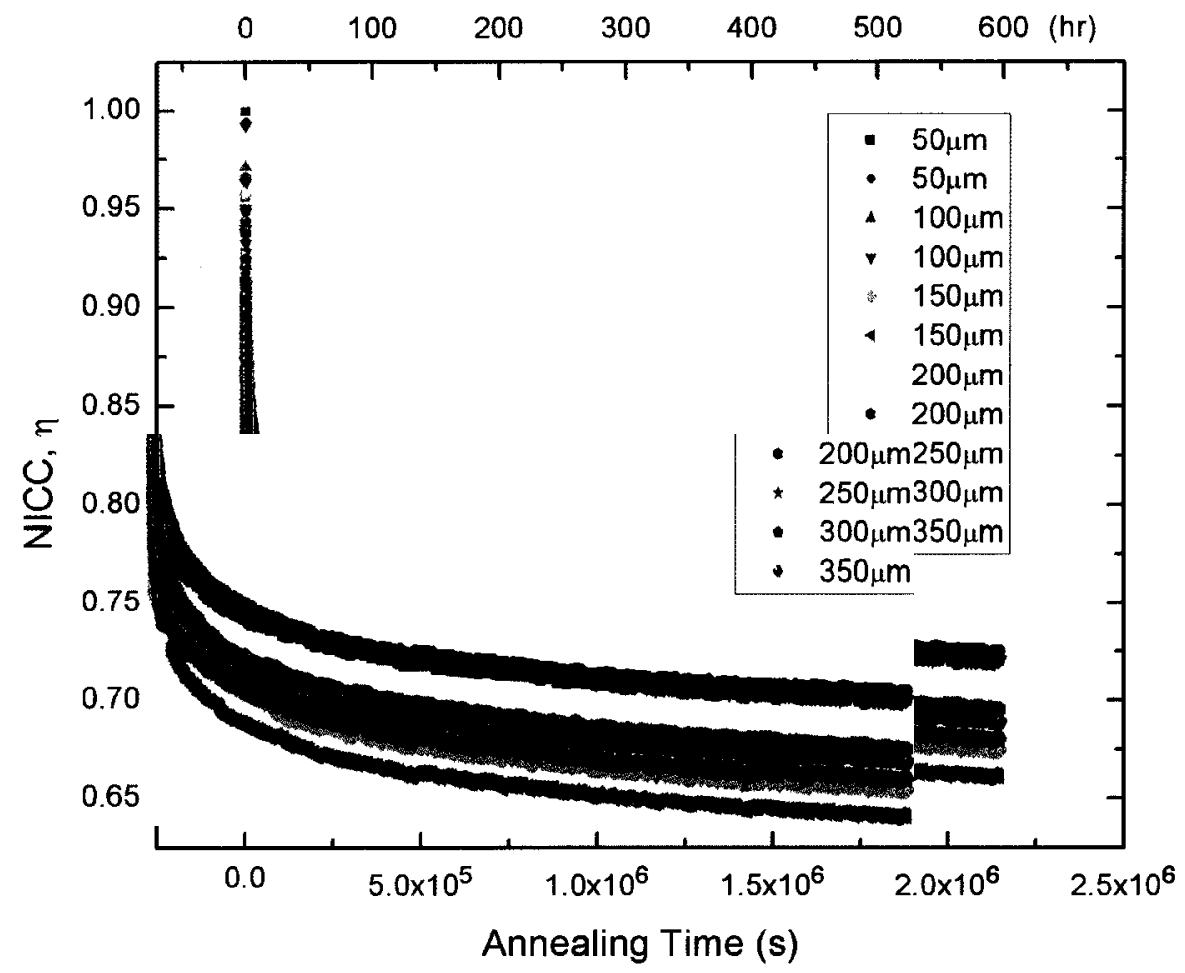

(a)

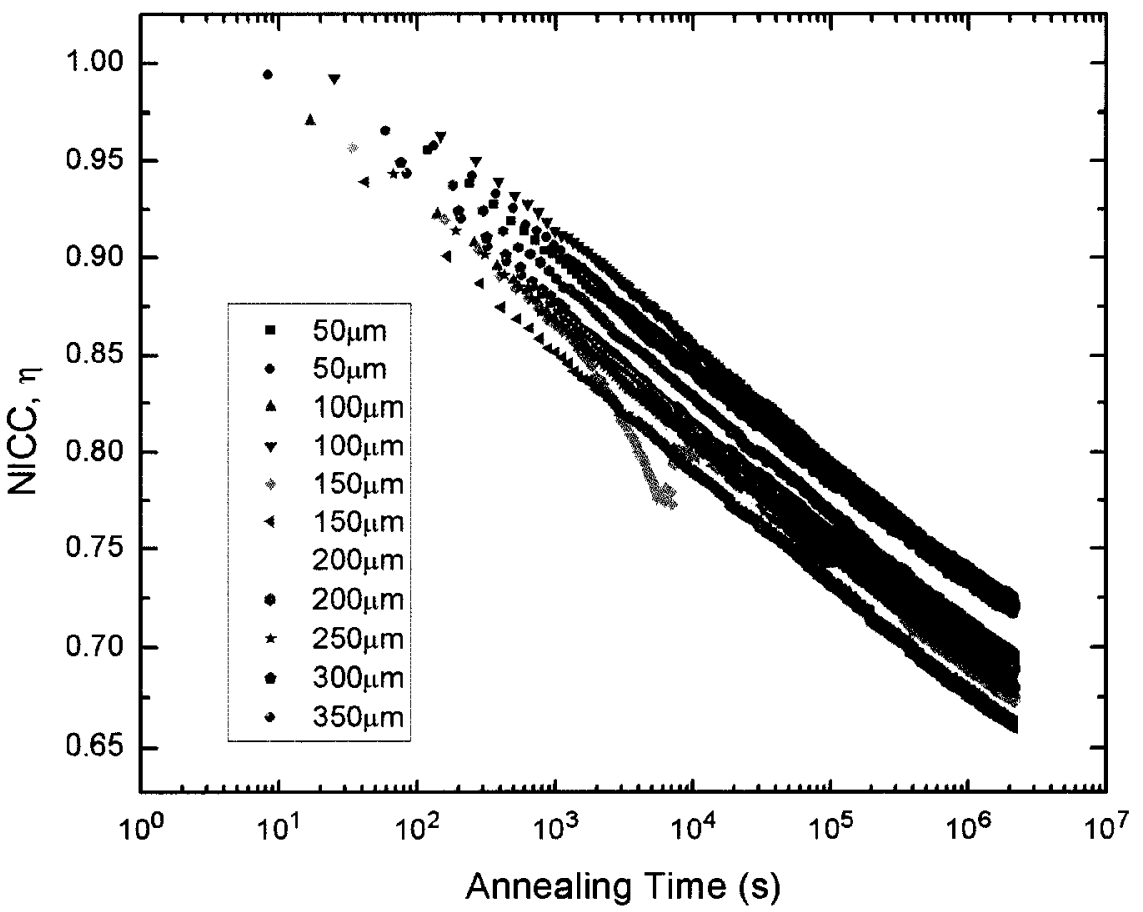

(b)

Fig 4.7 Isothermal decay of normalized integrated coupling constant (i.e. normalized refractive index modulation amplitude, normalized to the initial value at annealing temperature) for weak gratings as a function of annealing time at temperature of $300^{\circ} \mathrm{C}$ (a) linear time scale; (b) logarithmic time scale. 


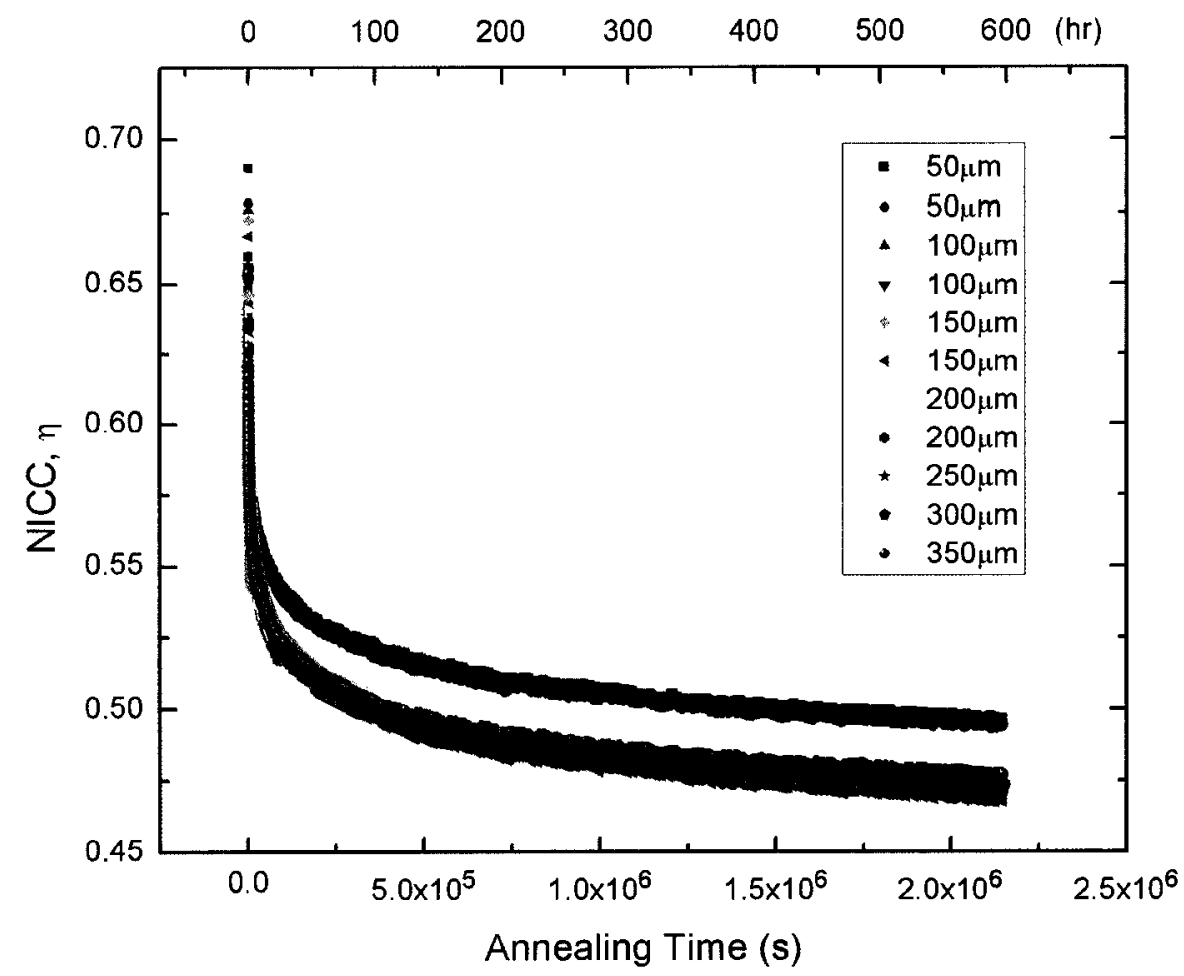

(a)

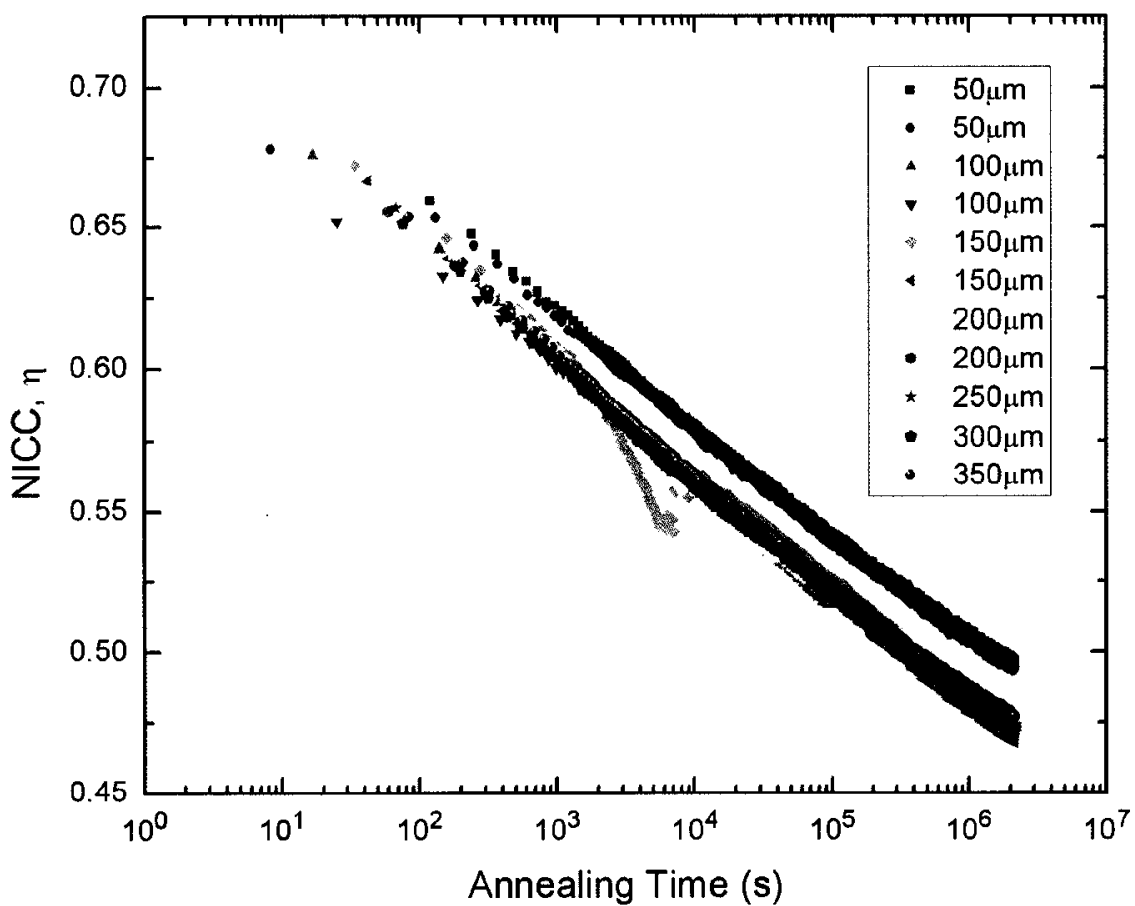

(b)

Fig 4.8 Isothermal decay of normalized integrated coupling constant (i.e. normalized refractive index modulation amplitude, normalized to the initial as-written value) for weak gratings as a function of annealing time at temperature of $300^{\circ} \mathrm{C}$ (a) linear time scale; (b) logarithmic time scale. 


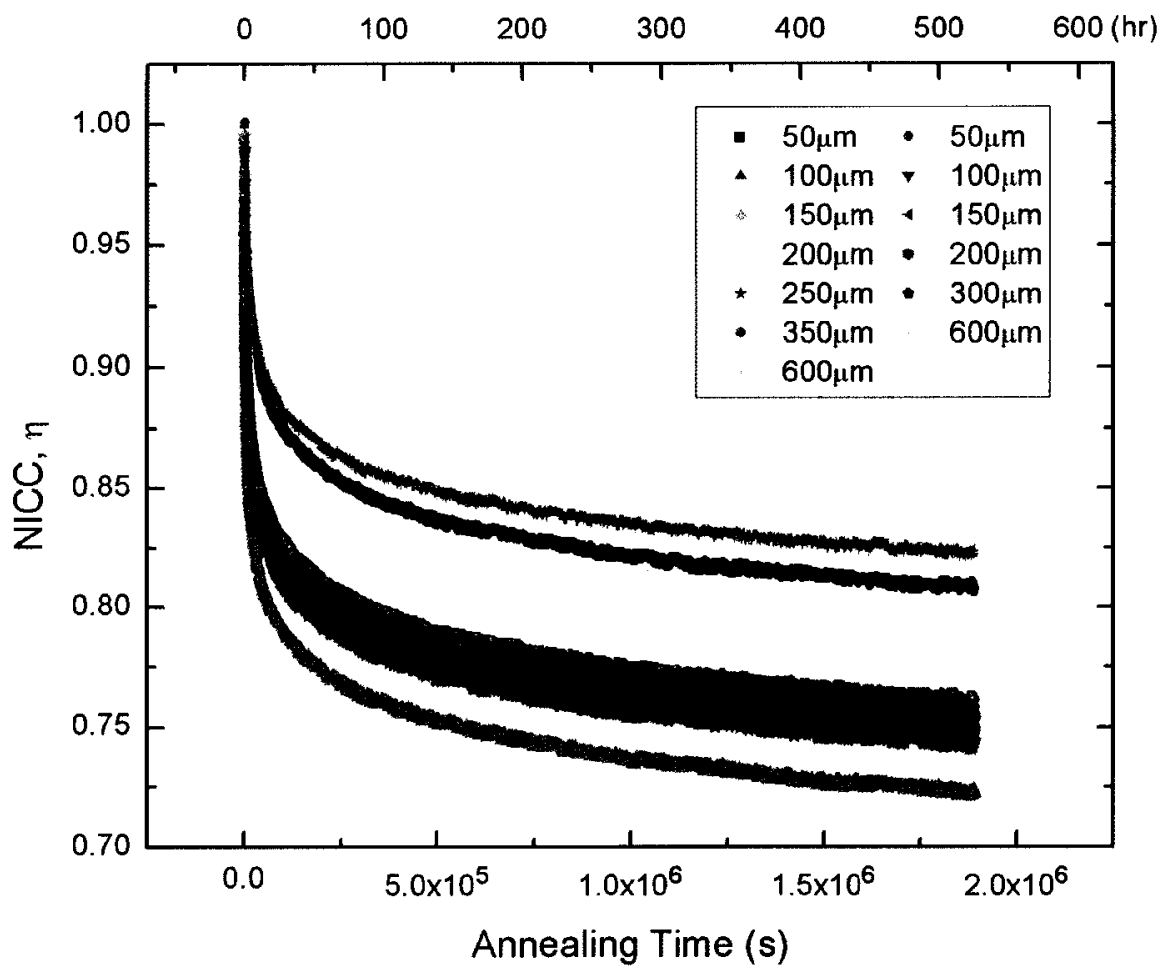

(a)

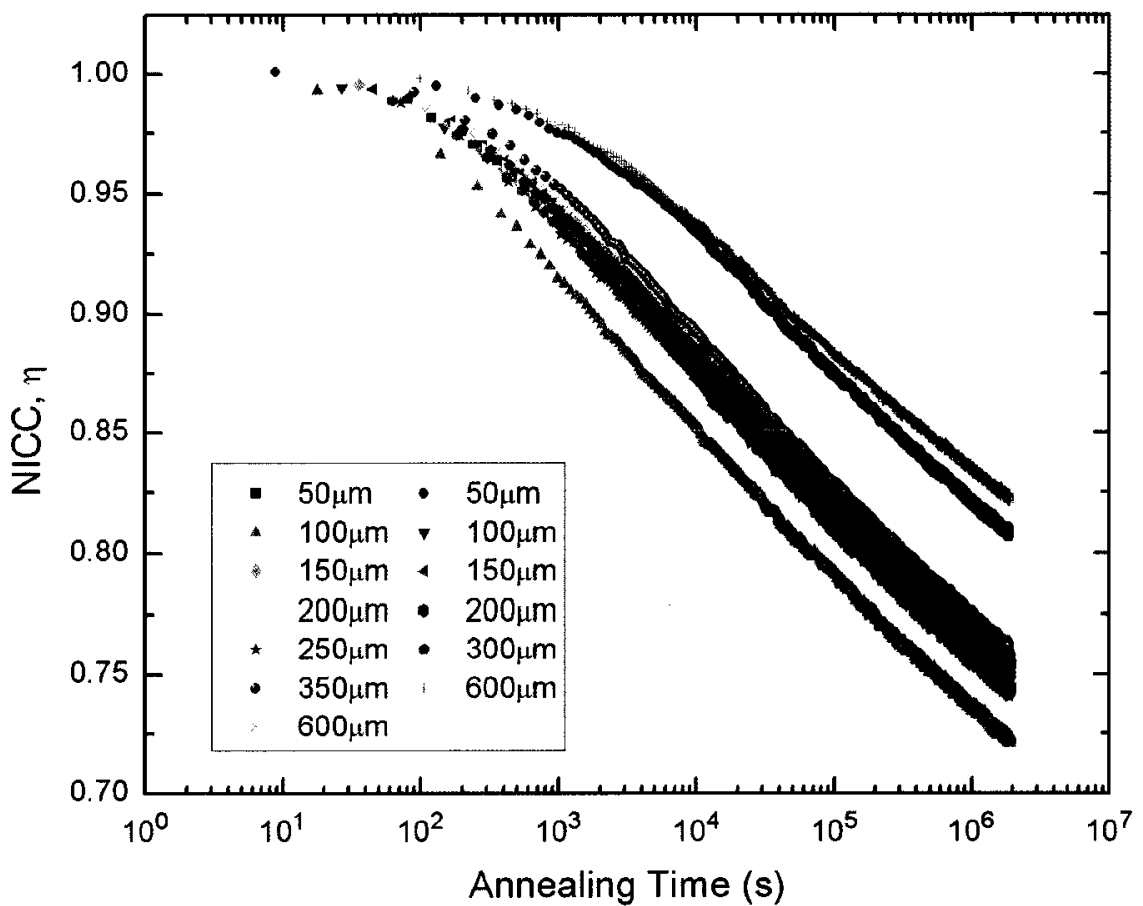

(b)

Fig 4.9 Isothermal decay of normalized integrated coupling constant (i.e. normalized refractive index modulation amplitude, normalized to the initial value at annealing temperature) for weak gratings as a function of annealing time at temperature of $250^{\circ} \mathrm{C}$ (a) linear time scale; (b) logarithmic time scale. 


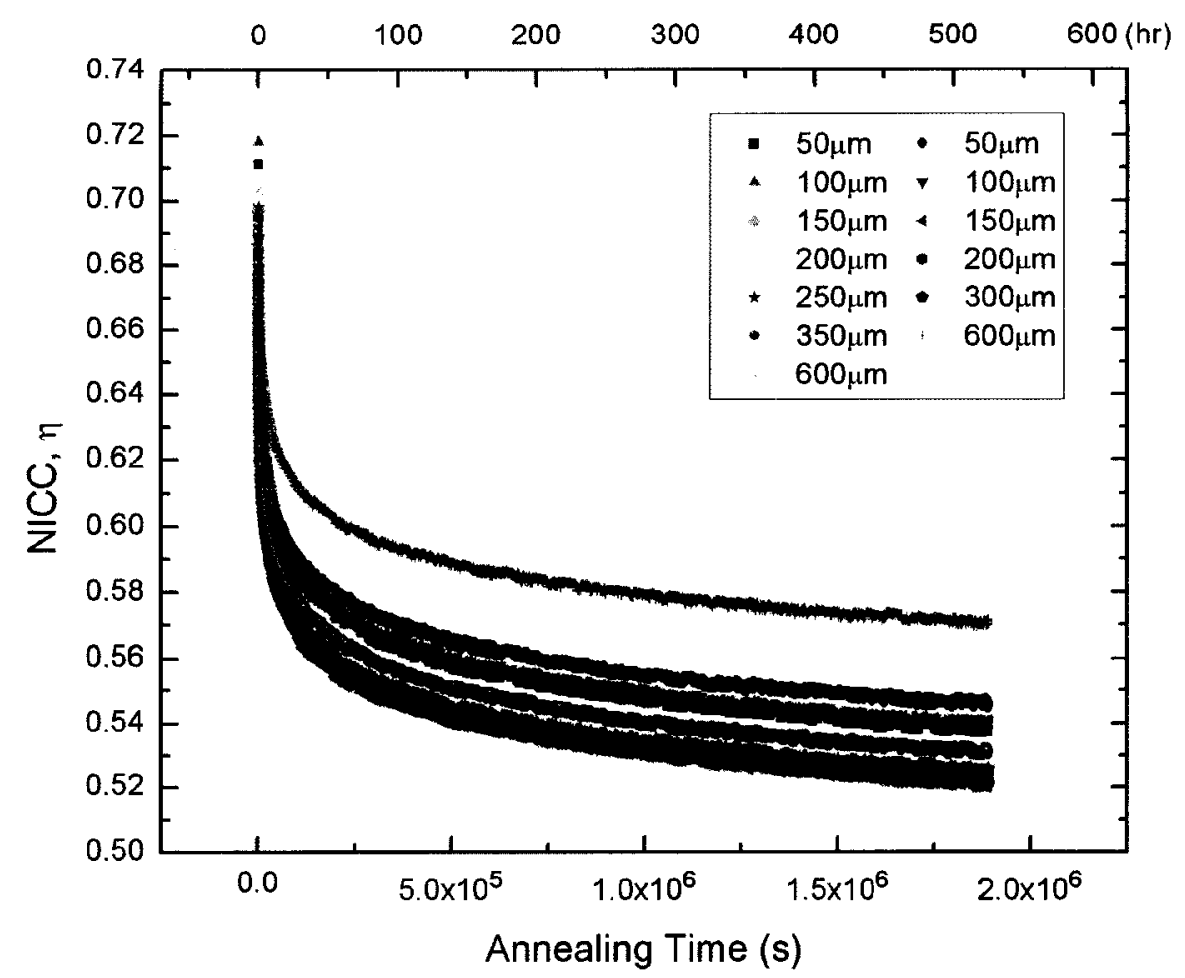

(a)

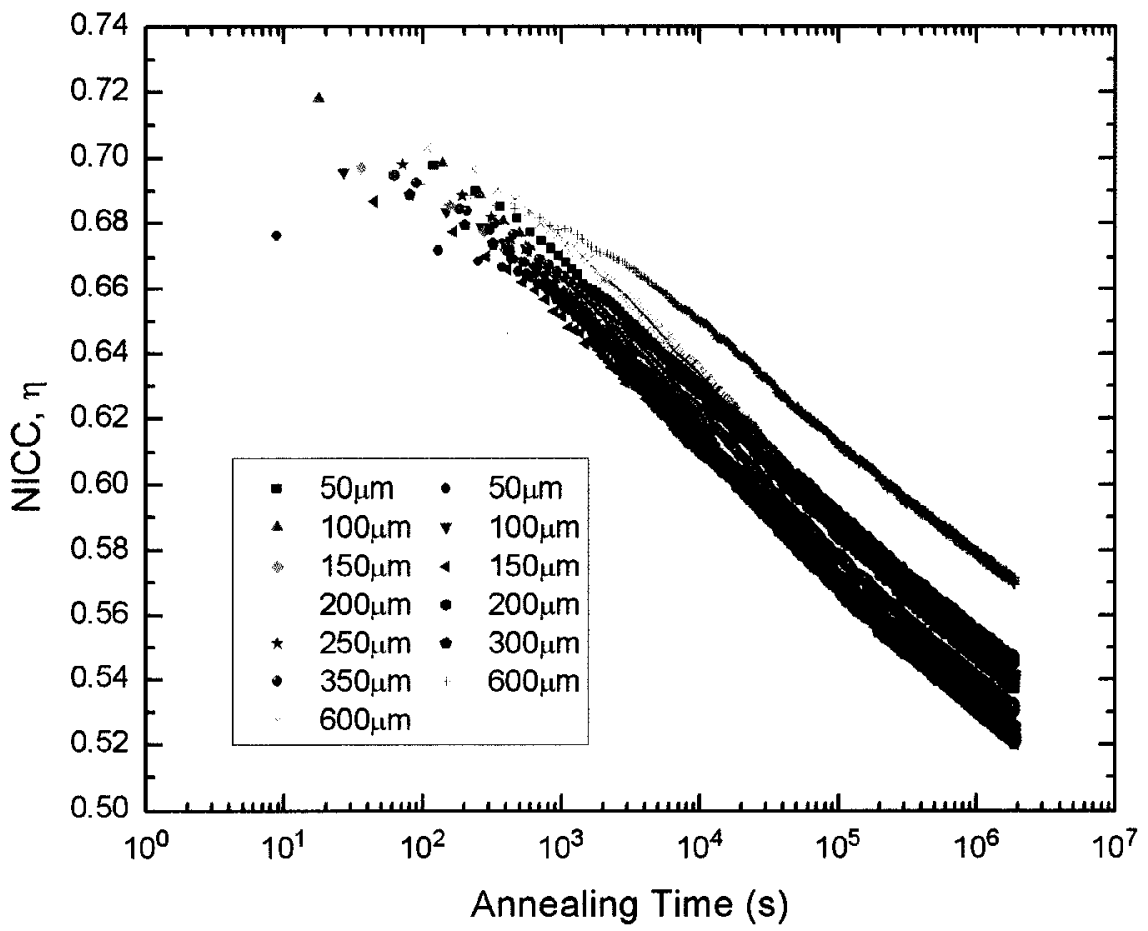

(b)

Fig 4.10 Isothermal decay of normalized integrated coupling constant (i.e. normalized refractive index modulation amplitude, normalized to the initial as-written value) for weak gratings as a function of annealing time at temperature of $250^{\circ} \mathrm{C}$ (a) linear time scale; (b) logarithmic time scale. 


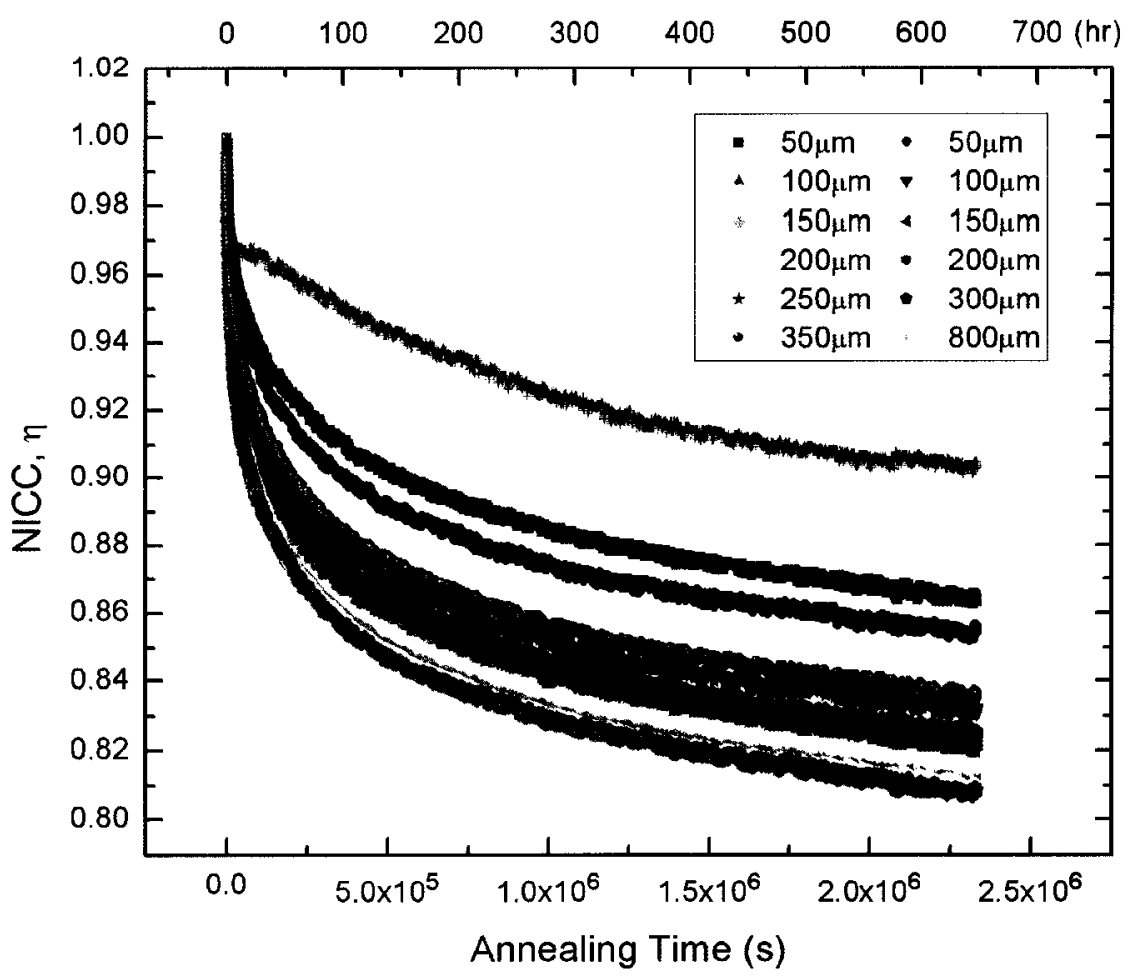

(a)

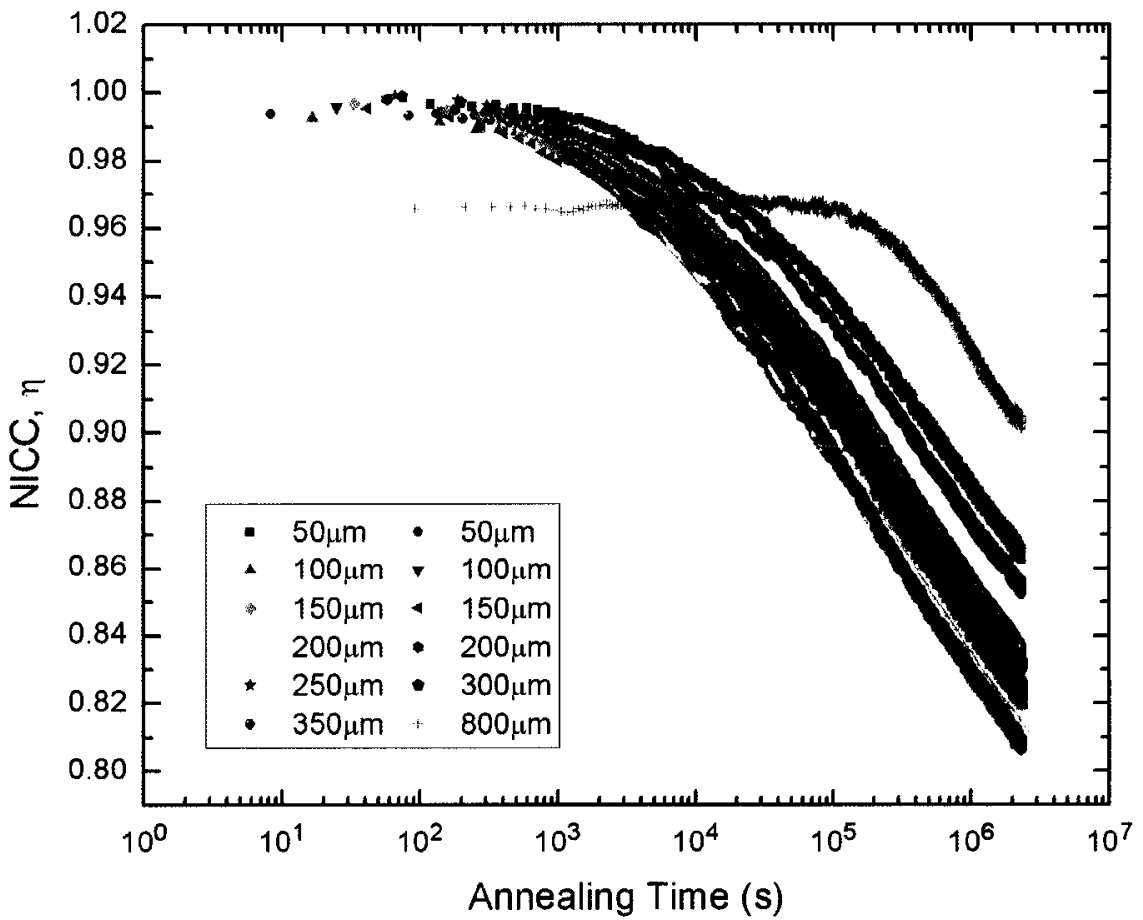

(b)

Fig 4.11 Isothermal decay of normalized integrated coupling constant (i.e. normalized refractive index modulation amplitude, normalized to the initial value at annealing temperature) as a function of annealing time at temperature of $200^{\circ} \mathrm{C}$ (a) linear time scale; (b) logarithmic time scale. 


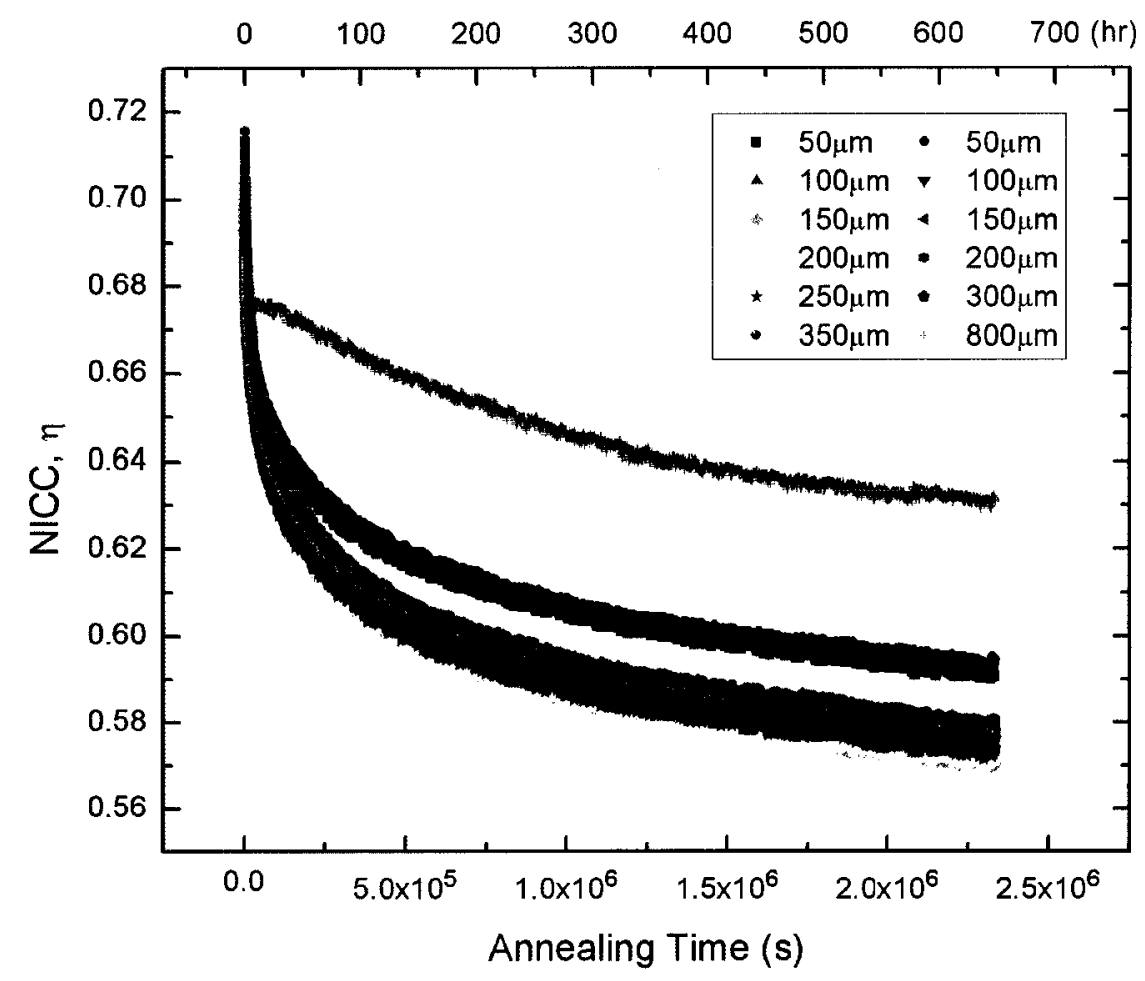

(a)

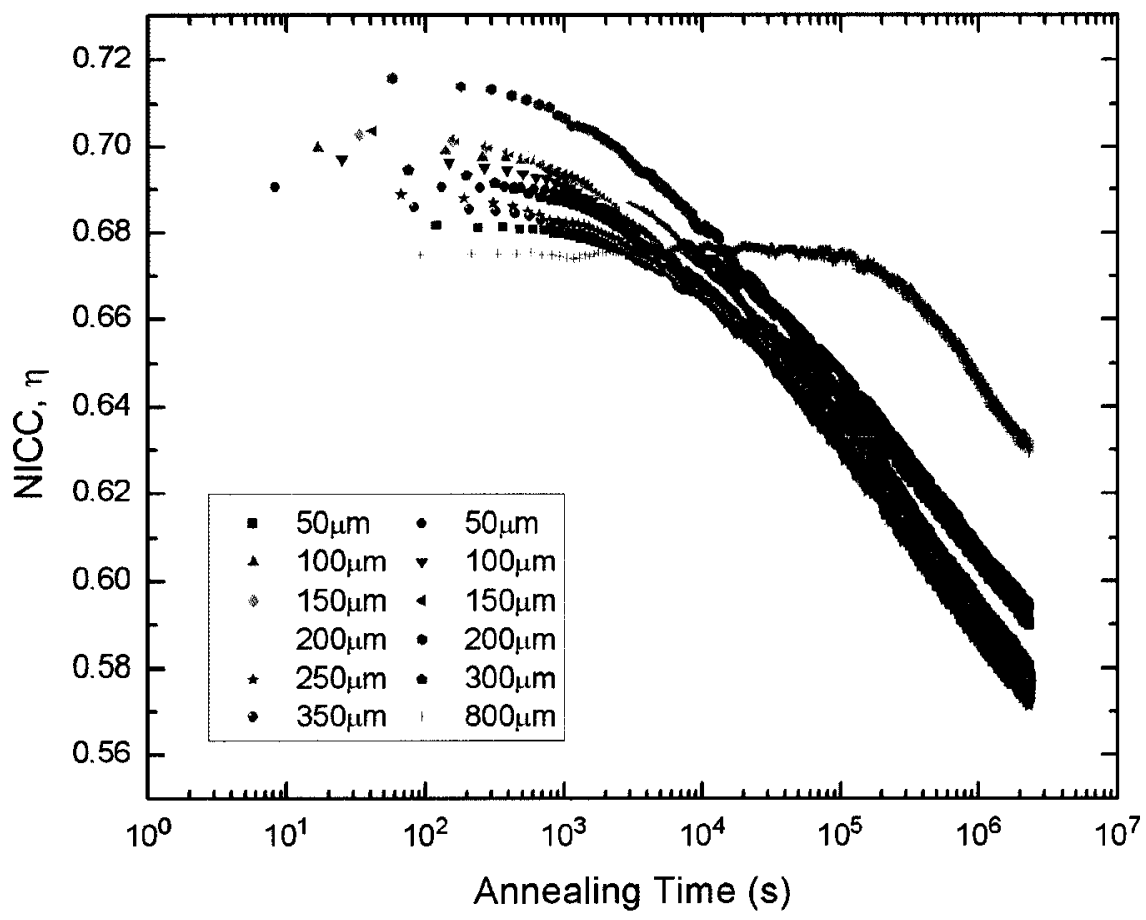

(b)

Fig 4.12 Isothermal decay of normalized integrated coupling constant (i.e. normalized refractive index modulation amplitude, normalized to the initial as-written value) for weak gratings as a function of annealing time at temperature of $200^{\circ} \mathrm{C}$ (a) linear time scale; (b) logarithmic time scale. 
As shown in these figures, within the accuracy of measurement, no big difference on thermal stability of grating can be observed for weak gratings inscribed at fiber/mask distance from $50 \mu \mathrm{m}$ to $600 \mu \mathrm{m}$. The only grating with apparently improved thermal stability is the grating written at $800 \mu \mathrm{m}$ as shown in the case of annealing temperature of $200{ }^{\circ} \mathrm{C}$. Unfortunately, no more gratings written at $800 \mu \mathrm{m}$ have been prepared for aging tests at other temperatures due to time limitations. In contrast to weak gratings, strong gratings written at $600 \mu \mathrm{m}$ have already demonstrated greater thermal stabilities than those with shorter writing distances. In summary, fabrication of the gratings at a relatively large fiber/mask distance can improve the thermal stability of grating reflectivity to a certain extent. The aging test results on these gratings will be discussed in depth in the next chapter.

\subsubsection{Bragg wavelength thermal evolution during accelerated aging}

In addition to the evolution of the NICC observed above, the evolution of the Bragg wavelengths for the gratings was also recorded in the course of the accelerated aging tests. As a result, the evolution of the DC refractive index change could be derived using the Bragg condition. However, different from the evolution of index modulation, the evolution of the DC refractive index change established from our results can not demonstrate a clear decay trend due to its large measurement error. This is mainly because the fluctuations in the annealing temperature during the aging test have influence only on the measurement of the Bragg wavelength and not on the transmission notch depth. Since the annealing temperature varied about $\pm 5^{\circ} \mathrm{C}$ around its desired value, an error of $\pm 0.05 \mathrm{~nm}$ was introduced to the measured Bragg wavelength. Because the decay 
amount of the DC index change of a grating is not very large $\left(\sim 20 \%\right.$ of $\left.2 \times 10^{-4}\right)$, the evolution of its Bragg wavelength does not follow a clear decay trend and falls within the measurement uncertainty.

\subsection{Model Fit Results}

As mentioned before, the simple power model $N I C C=a t^{b}$ has been applied to the thermal decay curves of all gratings. The fitting results of the pre-exponential factor "a" and the slope " $b$ " for both types of NICC (normalized to either the initial value at the annealing temperature or the as-written value) are listed in two separate tables. Tables 4.3 and 4.4 are the fitting results for the strong gratings, and Tables 4.5 and 4.6 are of the results for the weak gratings. As mentioned in Chapter 2, the final step to define the thermal decay characteristics of a grating is to develop a temperature dependence of the fitting factors "a" and "b". However, the decay parameters appear randomly distributed against the annealing temperature, and it was found that neither of the fitting factors " $a$ " and " $b$ " can be characterized by a well-defined temperature-dependent function. The validities of this simple power model and other existing standard models will be discussed in the next chapter in detail. 


\subsubsection{Fitting results of strong gratings}

\begin{tabular}{|c|c|c|c|c|c|c|}
\hline \multirow{2}{*}{$\begin{array}{c}\text { Distance } \\
(\boldsymbol{\mu} \mathbf{m})\end{array}$} & \multicolumn{2}{|c|}{ Decay fit for $200{ }^{\circ} \mathbf{C}$} & \multicolumn{2}{|c|}{ Decay fit for $250{ }^{\circ} \mathbf{C}$} & \multicolumn{2}{|c|}{ Decay fit for $300{ }^{\circ} \mathbf{C}$} \\
\cline { 2 - 7 } & $\mathbf{a}$ & $\mathbf{b}$ & $\mathbf{a}$ & $\mathbf{b}$ & $\mathbf{a}$ & $\mathbf{b}$ \\
\hline 600 & 1.16518 & -0.01724 & 1.15572 & -0.01919 & 1.10918 & -0.02262 \\
\hline 600 & 1.14982 & -0.01579 & 1.13643 & -0.01722 & 1.106876 & -0.02085 \\
\hline 350 & 1.214451 & -0.02578 & 1.137049 & -0.02722 & 1.016237 & -0.02599 \\
\hline 350 & 1.214502 & -0.02427 & 1.135507 & -0.02645 & 1.036603 & -0.02555 \\
\hline 100 & 1.20742 & -0.02284 & 1.128888 & -0.02804 & 1.012514 & -0.02653 \\
\hline 100 & 1.214829 & -0.02357 & 1.084258 & -0.02934 & 1.047633 & -0.02788 \\
\hline
\end{tabular}

Table 4.3 Fitting results for the NICC isothermal decay (normalized to the initial value at annealing temperature) of strong gratings

\begin{tabular}{|c|c|c|c|c|c|c|}
\hline \multirow{2}{*}{$\begin{array}{c}\text { Distance } \\
(\mu \mathbf{m})\end{array}$} & \multicolumn{2}{|c|}{ Decay fit for $200{ }^{\circ} \mathbf{C}$} & \multicolumn{2}{|c|}{ Decay fit for $250{ }^{\circ} \mathbf{C}$} & \multicolumn{2}{|c|}{ Decay fit for $300{ }^{\circ} \mathbf{C}$} \\
\cline { 2 - 7 } & $\mathbf{a}$ & $\mathbf{b}$ & $\mathbf{a}$ & $\mathbf{b}$ & $\mathbf{a}$ & $\mathbf{b}$ \\
\hline 600 & 0.857796 & -0.01724 & 0.840074 & -0.01919 & 0.805179 & -0.02262 \\
\hline 600 & 0.856634 & -0.01579 & 0.842383 & -0.01722 & 0.807875 & -0.02085 \\
\hline 350 & 0.889323 & -0.02578 & 0.80223 & -0.02722 & 0.750745 & -0.02599 \\
\hline 350 & 0.867539 & -0.02427 & 0.821815 & -0.02645 & 0.74467 & -0.02555 \\
\hline 100 & 0.832842 & -0.02284 & 0.801581 & -0.02804 & 0.718343 & -0.02653 \\
\hline 100 & 0.838957 & -0.02357 & 0.824333 & -0.02934 & 0.744986 & -0.02788 \\
\hline
\end{tabular}

Table 4.4 Fitting results for the NICC isothermal decay (normalized to the as-written value) of strong gratings 
4.3.2 Fitting results of weak gratings

\begin{tabular}{|c|c|c|c|c|c|c|}
\hline \multirow{2}{*}{$\begin{array}{c}\text { Distance } \\
(\mu \mathbf{m})\end{array}$} & \multicolumn{2}{|c|}{ Decay fit for $\mathbf{2 0 0}^{\circ} \mathbf{C}$} & \multicolumn{2}{c|}{ Decay fit for $\mathbf{2 5 0}^{\circ} \mathbf{C}$} & \multicolumn{2}{c|}{ Decay fit for $300{ }^{\circ} \mathbf{C}$} \\
\cline { 2 - 7 } & $\mathbf{a}$ & $\mathbf{b}$ & $\mathbf{a}$ & $\mathbf{b}$ & $\mathbf{a}$ & $\mathbf{b}$ \\
\hline 50 & 1.24513 & -0.02474 & 1.1495 & -0.02899 & 1.09609 & -0.02899 \\
\hline 50 & 1.248252 & -0.02575 & 1.191375 & -0.02689 & 1.100766 & -0.02881 \\
\hline 100 & 1.257588 & -0.02901 & 1.128737 & -0.03087 & 1.067463 & -0.03083 \\
\hline 100 & 1.263241 & -0.02922 & 1.159276 & -0.02988 & 1.147129 & -0.03205 \\
\hline 150 & 1.263669 & -0.03028 & 1.147352 & -0.02995 & 1.088089 & -0.03282 \\
\hline 150 & 1.253542 & -0.02978 & 1.153063 & -0.02943 & 1.072348 & -0.03326 \\
\hline 200 & 1.267601 & -0.03059 & 1.146 & -0.02952 & 1.034847 & -0.02776 \\
\hline 200 & 1.269797 & -0.0308 & 1.145893 & -0.02979 & 1.115221 & -0.03244 \\
\hline 250 & 1.271004 & -0.02893 & 1.144887 & -0.03002 & 1.077009 & -0.03173 \\
\hline 300 & 1.274529 & -0.02961 & 1.151373 & -0.02978 & 1.085883 & -0.03125 \\
\hline 350 & 1.268277 & -0.02836 & 1.163274 & -0.02942 & 1.0758 & -0.0307 \\
\hline 600 & & & 1.168396 & -0.02431 & & \\
\hline 600 & & & 1.155093 & -0.0295 & & \\
\hline 800 & 1.350565 & -0.02747 & & & & \\
\hline
\end{tabular}

Table 4.5 Fitting results for the NICC isothermal decay (normalized to the initial value at annealing temperature) of weak gratings

\begin{tabular}{|c|c|c|c|c|c|c|}
\hline \multirow{2}{*}{$\begin{array}{c}\text { Distance } \\
(\mu \mathbf{m})\end{array}$} & \multicolumn{2}{|c|}{ Decay fit for $200{ }^{\circ} \mathbf{C}$} & \multicolumn{2}{c|}{ Decay fit for $250{ }^{\circ} \mathbf{C}$} & \multicolumn{2}{c|}{ Decay fit for $300{ }^{\circ} \mathbf{C}$} \\
\cline { 2 - 7 } & $\mathbf{a}$ & $\mathbf{b}$ & $\mathbf{a}$ & $\mathbf{b}$ & $\mathbf{a}$ & $\mathbf{b}$ \\
\hline 50 & 0.851549 & -0.02474 & 0.817428 & -0.02899 & 0.756246 & -0.02899 \\
\hline 50 & 0.867358 & -0.02575 & 0.804864 & -0.02689 & 0.750993 & -0.02881 \\
\hline 100 & 0.886466 & -0.02901 & 0.816196 & -0.03087 & 0.743062 & -0.03083 \\
\hline 100 & 0.884263 & -0.02922 & 0.81094 & -0.02988 & 0.753586 & -0.03205 \\
\hline 150 & 0.890907 & -0.03028 & 0.80343 & -0.02995 & 0.763884 & -0.03282 \\
\hline 150 & 0.886194 & -0.02978 & 0.797091 & -0.02943 & 0.760725 & -0.03326 \\
\hline 200 & 0.894112 & -0.03059 & 0.808402 & -0.02952 & 0.716033 & -0.02776 \\
\hline 200 & 0.910815 & -0.0308 & 0.80526 & -0.02979 & 0.756847 & -0.03244 \\
\hline 250 & 0.876375 & -0.02893 & 0.809184 & -0.03002 & 0.749672 & -0.03173 \\
\hline 300 & 0.886182 & -0.02961 & 0.801568 & -0.02978 & 0.745098 & -0.03125 \\
\hline 350 & 0.875646 & -0.02836 & 0.811588 & -0.02942 & 0.744957 & -0.0307 \\
\hline 600 & & & 0.810223 & -0.02431 & & \\
\hline 600 & & & 0.8253 & -0.0295 & & \\
\hline 800 & 0.943581 & -0.02747 & & & & \\
\hline
\end{tabular}

Table 4.6 Fitting results for the NICC isothermal decay (normalized to the as-written value) of weak gratings 


\section{Chapter 5}

\section{Data Analysis and Discussion}

Based on the accelerated aging experiment results from Chapter 4, this chapter will discuss the dependence of the grating stability on the grating strength and the grating index contrast. The validity of the existing grating decay models will also be discussed. Finally, a comparison between our experiment and other experiments reported in literature will be done, and a possible mechanism for the enhancement effect of a larger DC component on the thermal stability of grating reflectivity will be suggested.

\subsection{Trends}

\subsubsection{Stability vs grating strength}

Based on the data obtained from the accelerated aging experiments for strong and weak gratings, the dependence of the annealing behavior on grating strength is investigated to provide a clear understanding about the complex nature of index change mechanism during the annealing process. In order to clarify the effect of the grating strength (herein means only the index modulation) on the thermal stability of a grating, a comparison will be done only on those gratings inscribed at fiber/phase mask distance of $100 \mu \mathrm{m}$, whose grating index contrasts are nearly 1 . This approach eliminates the potential impact of the average refractive index change on the thermal decay behavior.

Fig 5.1 illustrates the isothermal decay of the normalized index modulation of weak gratings at different annealing temperatures along those of strong gratings, all of which 


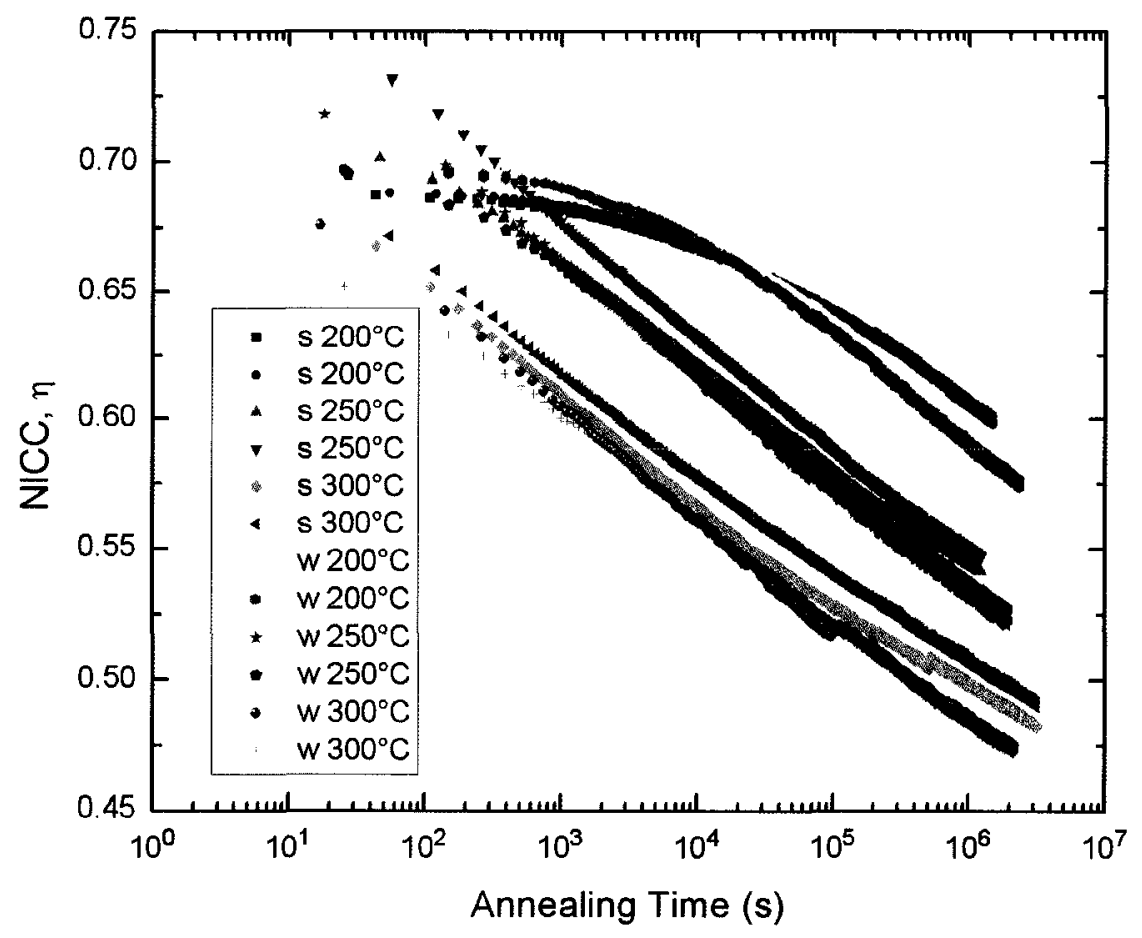

(a)

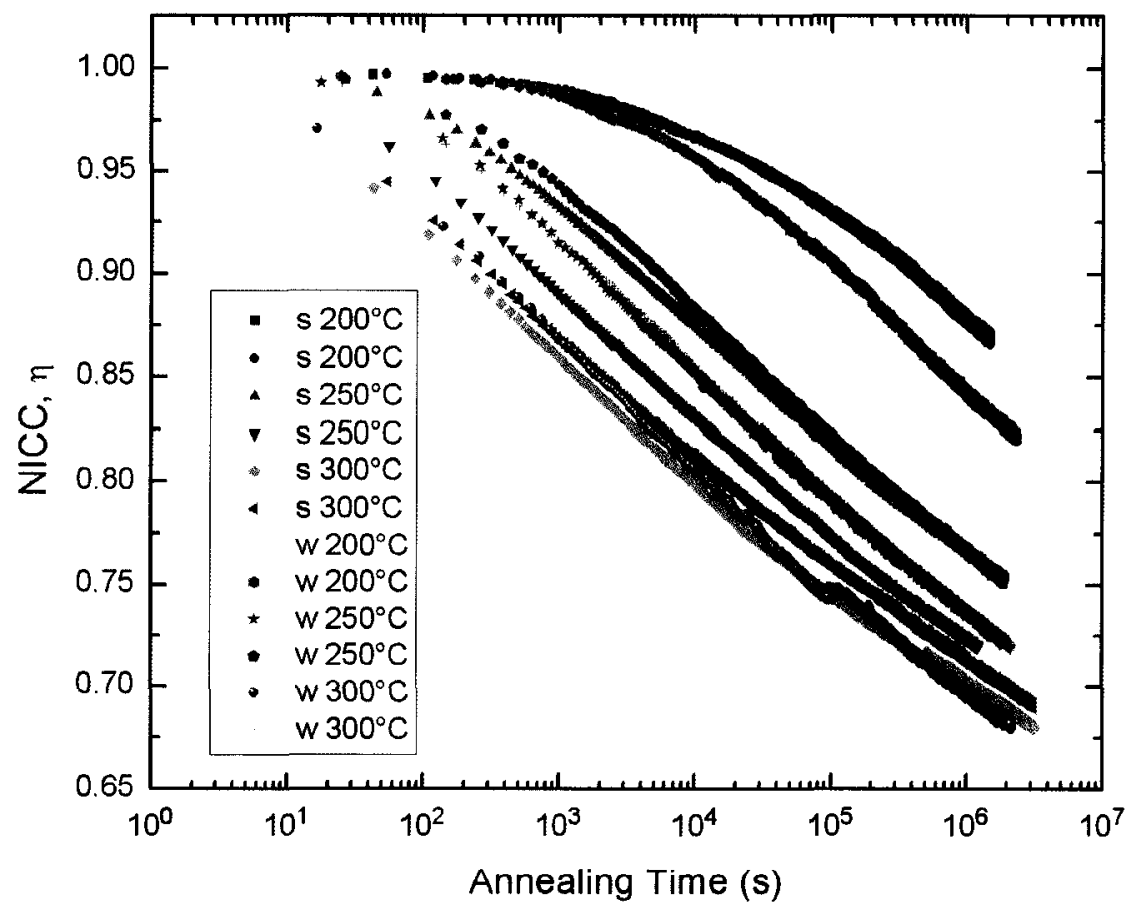

(b)

Fig 5.1 Thermal decays of strong and weak hydrogen-loaded pre-annealed gratings with writing distance of $100 \mu \mathrm{m}$ (a) NICC normalized to as-written value; (b) NICC normalized to initial value prior to annealing 
are written at a fiber/phase mask distance of $100 \mu \mathrm{m}$ and have approximately identical index contrast. In the legend shown in Fig 5.1, the symbols "s" and "w" denote strong and weak gratings respectively. Like in the previous chapter, the presented data is normalized by two different normalization factors, and the results are presented separately because the pre-annealing portion of the grating strength may play an important role in the subsequent thermal decay of the grating.

After an inspection on both Fig 5.1(a) and (b), it can be seen that the weak gratings show nearly same thermal stability as the strong gratings at annealing temperatures of $300^{\circ} \mathrm{C}$ and $250^{\circ} \mathrm{C}$. Only a slightly superior thermal stability of strong gratings over weak ones could be observed for the case of $200{ }^{\circ} \mathrm{C}$. The small difference of the thermal decay curves (around $1 \%$ in term of NICC at the end of annealing) between strong and weak gratings can be considered negligible compared to the larger statistical measurement error between two equivalent gratings annealed at the same temperature (NICC deviation of up to $2 \%$ is observed in the experiments).

The above observation demonstrates that the thermal stability of Bragg gratings written in hydrogen-loaded fibers does not significantly depend on the initial as-written index modulation in the range of $\sim 2.62 \times 10^{-4}$ to $\sim 6.57 \times 10^{-4}$. In contrast, some other papers reported that grating strength does affect thermal stability of fiber Bragg gratings, which are inscribed with UV pulsed irradiation on hydrogen-loaded standard telecommunication fibers [16] or nonhydrogenated boron-doped germanosilicate fibers [43]. It was observed that strong gratings had greater thermal stability than weak ones on both types of fibers (note that all hydrogen-loaded gratings had been stabilized using same pre-annealing process prior to the thermal annealing experiment.) According to the master aging curve 
model, this suggests that the strong gratings have different distribution of activation energy states from the weak grating. Strong gratings should have relatively larger population of electrons trapped at higher activation energy states. These higher energy trap sites are harder to overcome with thermal assistance compared to lower energy sites in order to cause a decay of the refractive index change. During annealing, weak gratings have a greater portion of lower energy trapping sites, which are subjected to thermal decay and a more rapid decay is observed.

However, reference [43] also observed the thermal decay of hydrogenated boron-doped germanosilicate FBGs, and no difference was found in the thermal stability between strong and weak gratings. This agrees with our observation. Therefore, it reminds us that the photosensitivity and the thermal decay of gratings depend on many factors, such as hydrogen loading, the dopants of the fiber, and the grating writing conditions. As far as our hydrogenated FBGs are concerned, the grating index modulation amplitude does not alter the thermal decay behavior of the gratings. During the grating fabrication, the long exposure of the hydrogen loaded fiber to a UV laser radiation will only excite carriers to reside at trap sites with the same activation energies as for the case of a short exposure. The above comparison between strong and weak gratings also helps us to further identify the origin of large deviations between two nominally identical gratings observed in the annealing experiment. This will be addressed detailed in next section.

\subsubsection{Stability vs fiber-mask distance}

As an initial goal of this research, we have attempted to develop an additional approach to improve the thermal stability of grating reflectivity. After carrying out several 
accelerated aging tests on hydrogen-loaded pre-annealed FBGs fabricated at various fiber/phase mask distances, the dependence of the grating's thermal stability on the writing distance (and hence on the grating index contrast) can be investigated. Besides determining the effect of writing distance on grating stability, this dependence also enables us to check whether small differences in fiber/mask distance during volume manufacturing of FBGs would impact the thermal stability of the gratings.

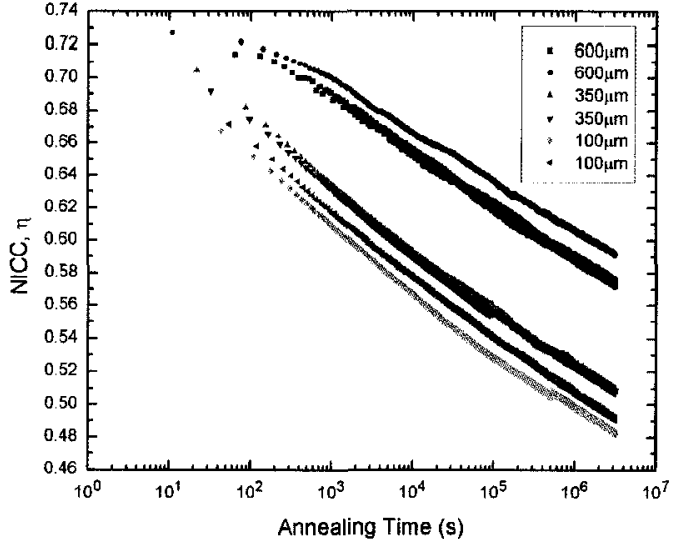

(a)

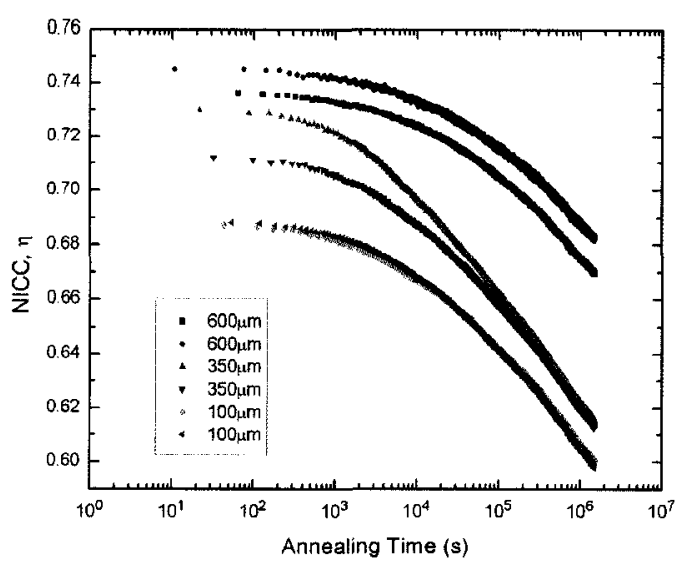

(c)

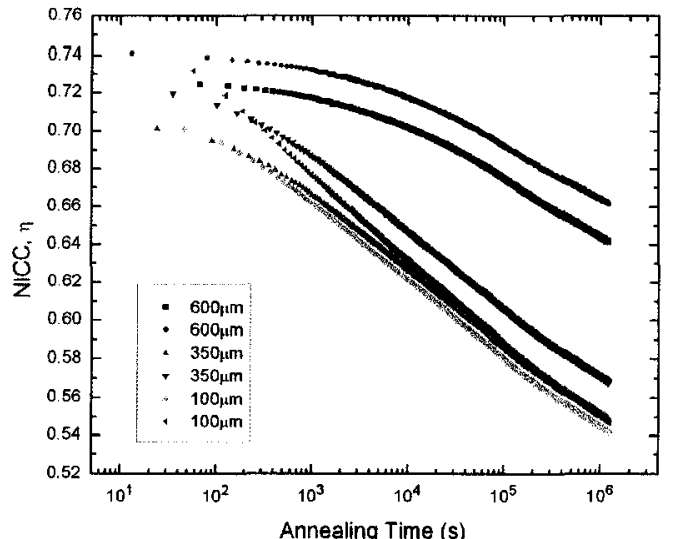

(b)

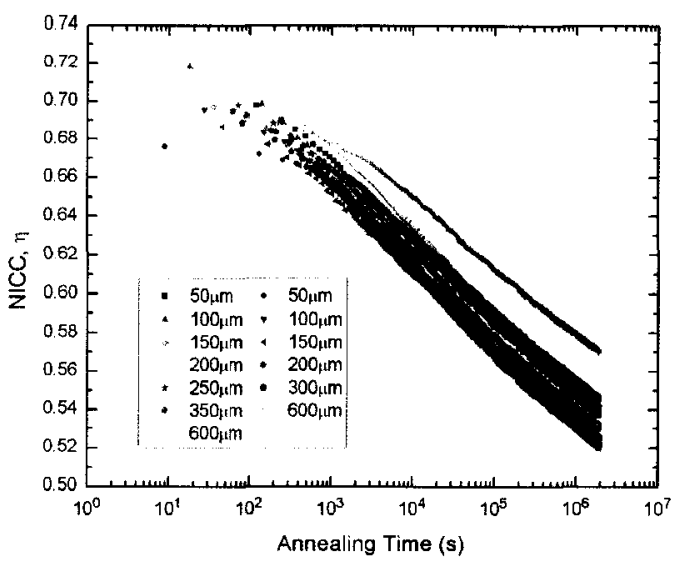

(d)

Fig 5.2 (replica) Thermal decays of hydrogen-loaded pre-annealed gratings written at various distances in terms of NICC normalized to as-written value (a) strong gratings at $300{ }^{\circ} \mathrm{C}$; (b) strong gratings at $250{ }^{\circ} \mathrm{C}$; (c) strong gratings at $200{ }^{\circ} \mathrm{C}$; (d) weak gratings at $250{ }^{\circ} \mathrm{C}$. 
For convenience, a number of thermal decay curves from Chapter 4 are replicated here in Fig 5.2. Three sets of curves for strong gratings annealed at different temperatures as well as a single set of curves for weak gratings annealed at $250^{\circ} \mathrm{C}$ were chosen as examples of the experimental data in order to allow us to compare the trends in the thermal decay of the gratings as a function of annealing time. This time, only NICC normalized to the aswritten value is chosen as the parameter to compare the thermal reliability of the gratings. Indeed, NICC normalized to grating strength prior the accelerated aging tests would not demonstrate clearly the impact of grating writing distance, since grating decay characteristics could be largely altered by the big variation in the amount of grating strength erasure during the pre-annealing process. This point will be explained in detail in the next section.

As illustrated in Fig 5.2 (a), (b) and (c), within the accuracy of measurement, strong hydrogen loaded FBGs written at the fiber/phase mask displacement of $600 \mu \mathrm{m}$ are apparently more thermally stable than their counterparts formed at $350 \mu \mathrm{m}$ and $100 \mu \mathrm{m}$ displacement with approximately same refractive index modulation for all gratings. For example, two gratings written at $600 \mu \mathrm{m}$ will be erased to $59.2 \%$ and $57.4 \%$ of their aswritten index modulation during annealing at $300{ }^{\circ} \mathrm{C}$ for 800 hours. In comparison, the same thermal annealing process would lead to a decrease in NICC to $51.0 \%$ and $50.9 \%$ for gratings of $350 \mu \mathrm{m}$, and $49.2 \%$ and $48.4 \%$ for those of $100 \mu \mathrm{m}$. This follows that a fairly large rise in the DC refractive index change comparatively increases the thermal stability of the grating reflectivity. Noting that grating index contrast at distance of 350 $\mu \mathrm{m}$ is still closer to that of $100 \mu \mathrm{m}$ as shown in Chapter 3 , it was expected that similar thermal stability had been observed for gratings of $350 \mu \mathrm{m}$ and $100 \mu \mathrm{m}$ since the increase 
in the DC refractive index change from $100 \mu \mathrm{m}$ to $350 \mu \mathrm{m}$ was not large enough to induce a noticeable improvement in the thermal stability of grating reflectivity.

However, as for weak hydrogen loaded fiber Bragg gratings in Fig 5.2 (d), the effect of the mean refractive index change on the enhancement of the thermal stability of grating reflectivity is not as prominent as that of strong gratings. At least, all of gratings inscribed at distances of $50 \mu \mathrm{m}$ to $350 \mu \mathrm{m}$ do not exhibit any difference on their thermal degradation curves during annealing at higher temperatures. Although increasing writing distance to $600 \mu \mathrm{m}$ probably strengthens grating thermal stability, this improvement is still smaller than the measurement error of the experiment so that one of the gratings written at $600 \mu \mathrm{m}$ has similar thermal decay behavior as gratings written at shorter phase mask / fiber distances.

In conclusion, a large amount of average refractive index change could improve thermal stability of grating reflectivity (solely determined by grating index modulation) in certain conditions. In order to achieve a pronounced improvement of thermal reliability, it is required that a large DC index change is formed inside grating. This finding removes the accuracy of the fiber-phase mask distance as one of the possible sources of variability in the thermal reliability of FBGs manufactured with excimer laser sources. The underlying mechanism of the enhancement effect of the average refractive index change will be investigated physically in Section 5.4. 


\subsection{Unexpected Results}

\subsubsection{Deviation of annealing data for two nominally identical gratings}

In this thesis work, we analyze the thermal decay of pre-annealed gratings by plotting the grating index modulation evolution as a function of the annealing time. The grating index modulation is normalized using two different normalization schemes - to its initial value prior to annealing (generally used in other researches), and to the as-written value of the modulation. However, dissimilar grating decay patterns in the two figures used to plot these different normalization schemes lead to confusion about the correctness of the two normalized index modulations. For instance, some pairs of similar gratings exhibit identical thermal decay curves in both figures, or some pairs have overlapping thermal decay patterns in one normalization figure but deviating in another figure. Additionally, some pairs completely separate from each other at the beginning of the thermal annealing process, and converge nicely to one decay curve for long annealing time. It is unclear whether the different decay characteristics of two similar gratings originate from the fabrication process or the pre-annealing procedure, or even the statistical error during the measurements.

During fabrication of fiber Bragg gratings, the fluctuation of the grating writing condition (fluence per pulse, or spatial coherence of UV laser) or the small variation of the aswritten index modulation formed in the fiber may contribute to different thermal decay behaviors of the grating. Based on the finding about the dependence of the thermal stability on the grating strength in Subsection 5.1.1, the effect of the small variation of the as-written index modulation on the thermal decay behavior of the grating could be eliminated. An increase in the grating index modulation from $\sim 2.62 \times 10^{-4}$ to $\sim 6.57 \times 10^{-4}$ 
does not alter grating decay pattern significantly, therefore the as-written index modulation of the gratings varying within the range of $\pm 3.00 \times 10^{-5}$ during fabrication apparently should not be responsible for the observed relatively large deviation in the measurement data for two equivalent gratings annealed at the same temperature.

As for the concern on the fluctuation of grating writing condition, efforts have been made to minimize this fluctuation by inscribing the gratings continually in order to keep the writing conditions consistent. Additional accelerated aging tests have been performed on hydrogen loaded FBGs without pre-annealing, which are formed at $100 \mu \mathrm{m}$ with similar grating index change as the strong gratings in our thermal annealing experiment. Before launching thermal annealing at $300{ }^{\circ} \mathrm{C}$, these gratings have been kept at room temperature for several days to allow residual hydrogen inside fiber to diffuse out. The thermal decay behavior of these hydrogen-loaded FBGs is shown in Fig 5.3. It is apparent that all thermal decay curves of these gratings overlap very well. This further confirms that all grating samples employed in each series in our thermal annealing experiment could be considered as equivalent in terms of their thermal stability after their fabrication.

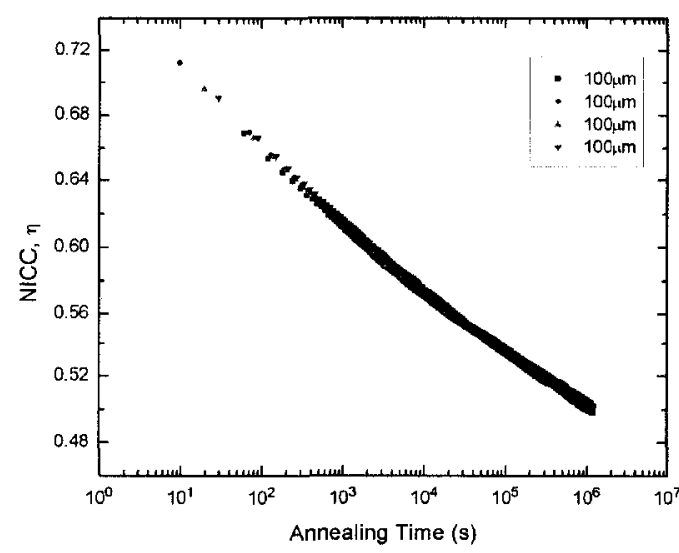

Fig 5.3 Thermal decay curves for four similar hydrogen-loaded gratings written at 100 $\mu \mathrm{m}$ during annealing at $300{ }^{\circ} \mathrm{C}$. 
The above discussion suggests that the difference in thermal decay patterns exhibited by two nominally equivalent pre-annealed FBGs is related to loose control of the preannealing procedure or the measurement errors. As mentioned in Chapter 3, the quick annealing technique utilized in the pre-annealing procedure is a process under loose control, which results to a relatively large variation of the amount of grating strength erasure for each grating (between $23.25 \%$ and $30.09 \%$ in Table 4.1 ). There could also be a big variation in the characteristics of the distribution of the trapped site energy states due to the different thermal histories during this quick annealing step. Consequently, the subsequent thermal decay of gratings would be altered by this variance. As an example, the data obtained during the thermal annealing of strong gratings at $200{ }^{\circ} \mathrm{C}$ in Chapter 4 is replotted in Fig 5.4. Both kinds of NICC are shown for comparison.

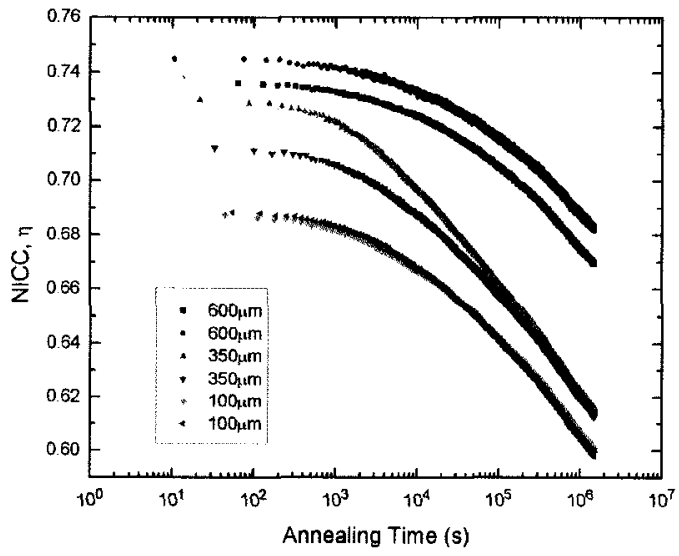

(a)

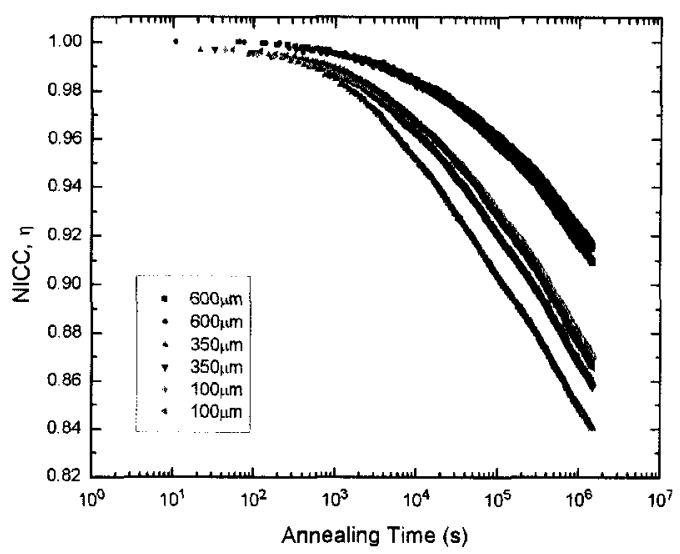

(b)

Fig 5.4 (replica) Thermal decays of hydrogen-loaded pre-annealed gratings written at various distances at $200{ }^{\circ} \mathrm{C}$ (a) in terms of NICC normalized to as-written value; (b) in terms of NICC normalized to initial value prior to annealing.

For writing distances $100 \mu \mathrm{m}$ and $350 \mu \mathrm{m}$, the thermal decay patterns of equivalent gratings converge to single curve at long annealing times, although they may be separated from each other at the beginning of annealing process. Especially for the ideal case at 100 
$\mu \mathrm{m}$, the overlapping thermal decay curves of two similar gratings in both figures reveal that these two gratings are equivalent in their thermal stability, both in terms of the aswritten and the pre-annealed NICC. For the two samples written at $600 \mu \mathrm{m}$, their thermal decay curves demonstrate nearly the same pattern with a $1 \%$ difference in the as-written NICC throughout the entire annealing process. It is plausible to suppose these two gratings follow the same thermal decay curves, and that the small deviation in the normalized refractive index modulation is a systematic error in grating strength introduced during either the writing (i.e. normalization factor) or the annealing (i.e. experimental data) process.

Surprisingly, the figure of NICC normalized to the initial value prior to annealing demonstrates that the gratings at $100 \mu \mathrm{m}$ have superior stability than those at $350 \mu \mathrm{m}$. This is contrary to the observed results in the figure of NICC normalized to the as-written grating strength. Upon Careful inspection of the beginning part of the thermal decay in the figure, it is clear that a much greater portion of the grating strength of the $100 \mu \mathrm{m}$ gratings has been erased prior to the accelerated aging compared to the gratings at 350 $\mu \mathrm{m}$. This means that more defect sites with low activation energy have been removed from the gratings written at $100 \mu \mathrm{m}$ phase mask / fiber distance. As a result, these stabilized gratings at $100 \mu \mathrm{m}$ have a greater improvement in thermal stability than those at $350 \mu \mathrm{m}$ after the pre-annealing process, although without any pre-annealing process the as-written gratings of $100 \mu \mathrm{m}$ may be slightly less stable than those of $350 \mu \mathrm{m}$. This large pre-annealing portion contributes to an increased stability exceeding the enhancement effect from the increased writing distance. 
It should be noted that the concern of using two normalization factors does not exist in analysis of annealing data for non hydrogen loaded gratings, which are not generally subjected to any pre-annealing process before launching the thermal annealing experiments.

\subsubsection{Large deviation in a single thermal annealing data}

All the thermal decay datasets of gratings obtained in our work are very smooth throughout the entire annealing process, except two samples in the weak gratings group that have shown a large deviation in their decay curves. This strange behavior is only observed in a single thermal decay experiment, where more than 10 weak gratings are annealed at $300{ }^{\circ} \mathrm{C}$. This experiment result is plotted again in Fig 5.5. As shown in the figure, two of the gratings under test show a large deviation in the NICC from their context evolutions, which apparently interrupt the continuity of the grating's thermal decay. The deviations of NICC observed in two samples are $7.3 \%$ and $2.5 \%$ respectively, which is much larger than the statistical error of about $2 \%$ reported in the last subsection. No valid explanation is found to account for the big error in the normalized refractive index modulation in these specific thermal decay datasets. However, by examining other papers investigating thermal degradations of fiber Bragg grating, it seems that a similar or an even greater measurement error exists in other reports as well. For instance, an error up to $8 \%$ was found in data obtained for thermal annealing in reference [44]. It has to be noted that although a big distortion has occurred in the course of the annealing, the decay curve of the grating still returns back to its normal shape and clearly demonstrates an evolution trend. 


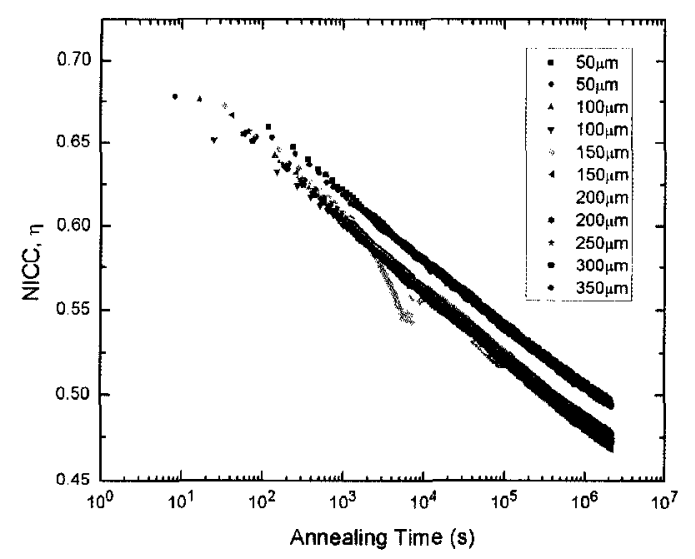

Fig 5.5 (replica) Thermal decays of NICC (normalized to as-written value) for weak gratings at $300^{\circ} \mathrm{C}$

\subsection{Validity of the decay models}

This section will discuss the validity of all existing standard accelerated aging models, mentioned in Chapter 2, for the case of hydrogen loaded PRE-ANNEALED FBGs. Although a simpler power law model has been chosen to analyze all of thermal experimental data before and this model is found to be capable of characterizing thermal degradation data for long annealing times, an apparent discrepancy between the experimental data and fitting result is observed at the beginning of the isothermal annealing (especially at lower annealing temperatures). However, after attempts of applying other standard thermal decay models have been made, our detailed experimental results indicate that none of the existing models can describe accurately the thermal decay behavior of our pre-annealed gratings. 


\subsubsection{Validity of the Erdogan power law model, the log time model, and the simple power model}

As summarized in Chapter 4, thermal decay of grating strength typically comprises of two different regimes: short-term decay and long-term decay, which are characterized by different rates of change in NICC as a function of the logarithm of the annealing time. For the sake of a clear demonstration of the fitting properties of each model in both regimes, three typical thermal decay curves are used as examples to be fitted with different decay models. Fig 5.6 depicts the thermal degradation of NICC (normalized to the initial value prior to annealing) for strong gratings in 3 cases, along with their fitting results using the Erdogan power model, the log time model, and the simple power model, which are expressed as equations (5.1), (5.2) and (5.3), respectively (see detailed in Chapter 2).

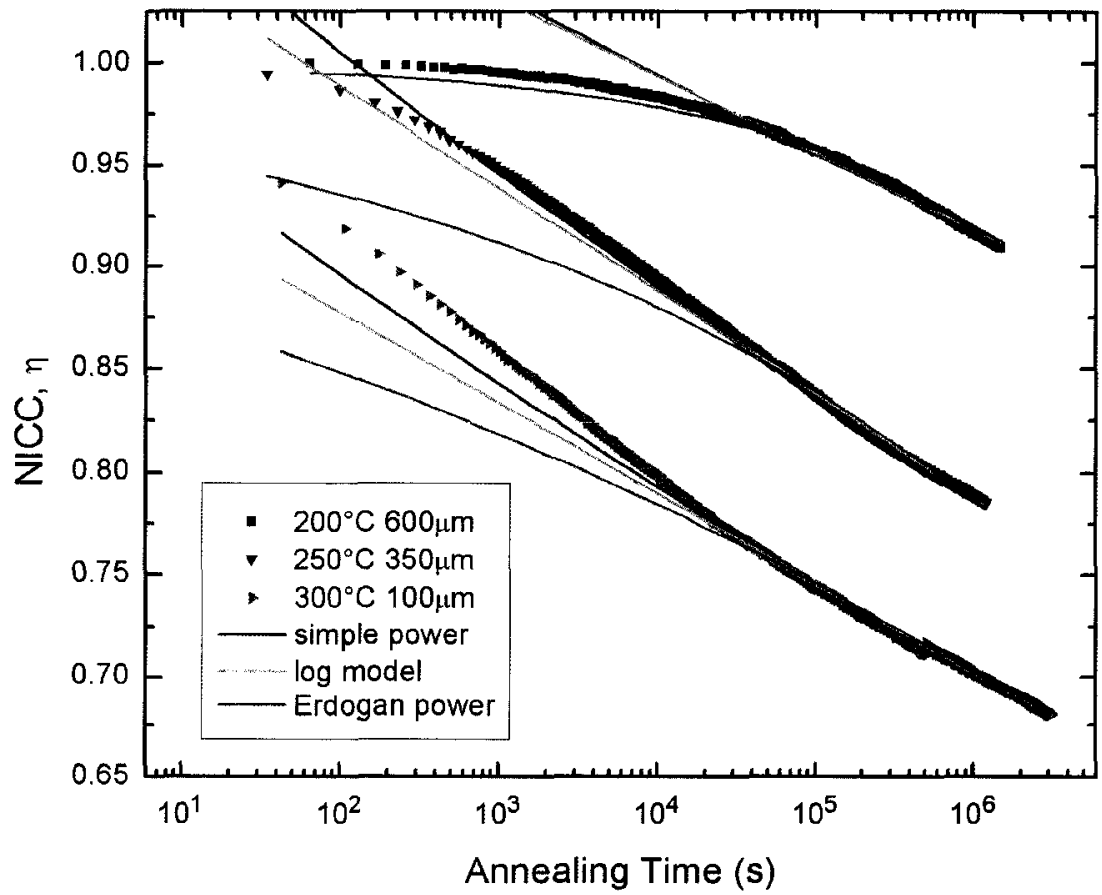

Fig 5.6 Thermal decay of the NICC for strong gratings fitted with the simple power model, the log time model, and the Erdogan power model. 


$$
\begin{array}{ll}
\eta=\frac{1}{1+A\left(t / t_{1}\right)^{\alpha}} & \begin{array}{ll}
1-k \log (t / \tau) & \text { for } t>\tau \\
1 & \text { for } t \leq \tau
\end{array} \\
\eta= \begin{cases}a t^{b} & \text { for } t>\tau=a^{-1 / b} \\
1 & \text { for } t \leq \tau=a^{-1 / b}\end{cases}
\end{array}
$$

For simplicity, the plotted fitting functions for the simple power model and the log time model do not include the $t \leq \tau$ portion of the functions. As shown in the Fig 5.6, all decay models fit reasonably well to the experimental data at long annealing times, where the decay curves obtained for all hydrogen-loaded FBGs are nearly straight lines with respect to the logarithmic time scale, although each model exhibits slightly different slope of thermal decay in this regime (which may lead to a substantial deviation between them in the subsequent thermal decay). However, all decay models diverse from each other at the beginning of accelerated aging test, and are largely separated from the experimental data set at all annealing temperatures.

For the case of $200{ }^{\circ} \mathrm{C}$ in this figure, the thermal decay curve have a relatively large part of short-term decay, which could be best fitted by the Erdogan power law model among the three models. This is attributed to the feature of the Erdogan power function at the beginning of decay, which presumes the initial value of NICC being equal to 1 inherently in the function and hence enables us to fit to some extent the curvy pattern of grating's short-term decay. However, apparent separation between the fitting curves of the Erdogan power function and the experimental data in the short-term decay regime is observed at higher temperatures, especially at $300{ }^{\circ} \mathrm{C}$, where a deviation of up to $8.3 \%$ 
was measured between the fitted and the experimental values at the start of the thermal annealing process.

In contrast, due to the mathematical features of the simple power and the log function models, namely that both will go to infinity at time zero, the fit based on these two models is larger than the measured value of the NICC during the short term decay for the $200{ }^{\circ} \mathrm{C}$ case. Although the starting time of decay parameter $\tau$ is introduced in both models to force the NICC to be equal to 1 at short annealing times, the deviation between these models and the annealing data is still inevitable in the regime of short term decay where the measured NICC is always less than 1 as shown in the figure. Nonetheless, for the case of higher temperatures, at which the long term decay of grating is rapidly approached, both models are superior to the Erdogan power model in describing the decay characteristics, and the simple power model is better than the log time model.

A comprehensive analysis of the thermal degradation characteristics of a grating under test requires the development of a temperature dependence of the fitting coefficients after a specific decay model is applied to the annealing data of the grating at several different temperatures. Once the temperature dependence is established, a prediction of the grating lifetime at its typical operating temperature (lower than $70^{\circ} \mathrm{C}$ ) can be made. However, the investigation of the fitting coefficients obtained for each model and their variation as a function of the annealing temperature further confirms that all of above models are not applicable to describe the thermal decay characteristics of grating reflectivity. 


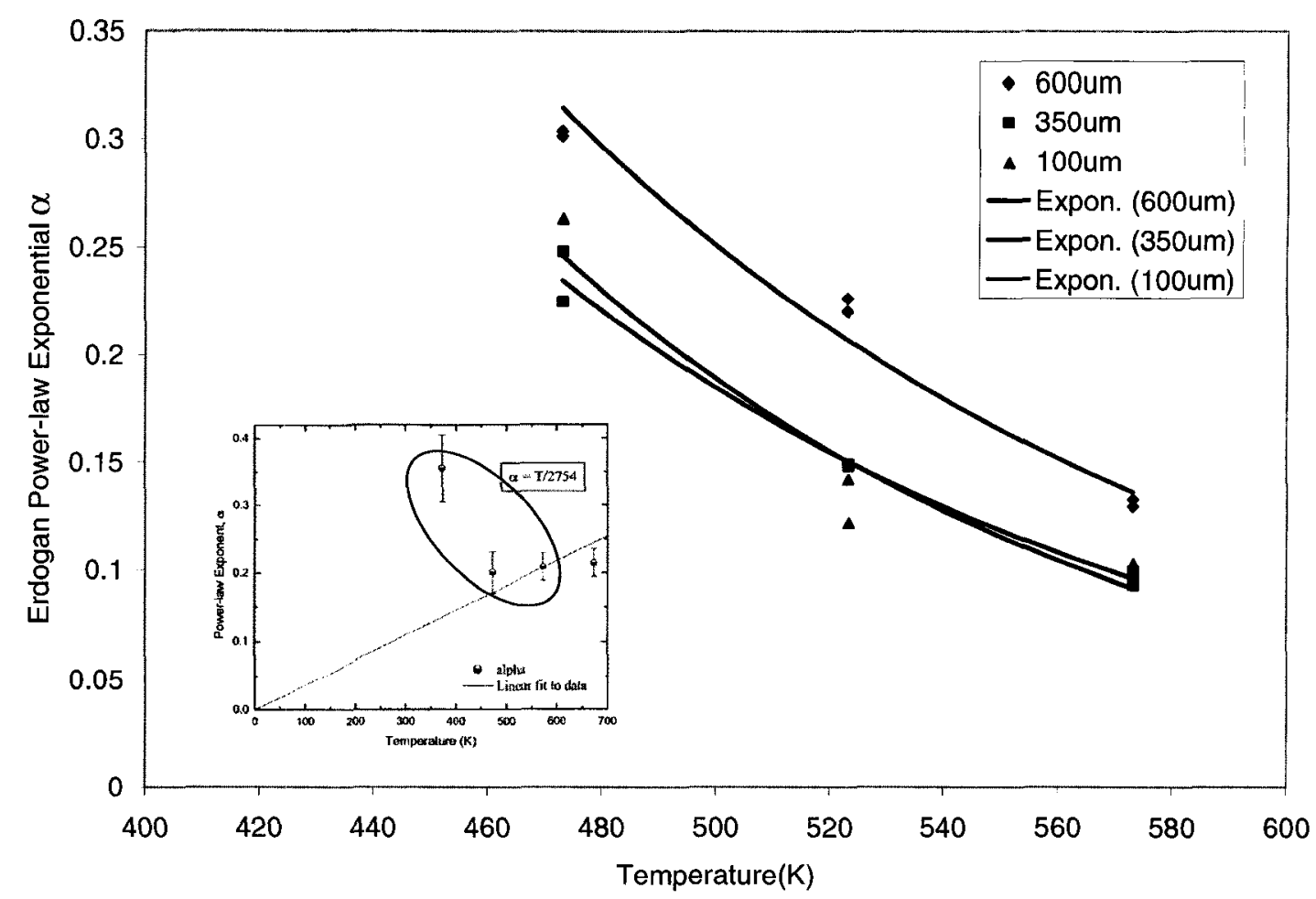

Fig 5.7 The temperature dependence of Erdogan power-law exponent factor $\alpha$ (Inset shows the temperature dependence of $\alpha$ from ref [32] for comparison).

The above figure shows the dependence of the exponent $\alpha$ on temperature, which is supposed to follow a linear relationship passing through the zero at temperature of $0 \mathrm{~K}$ based on the Erdogan power-law model [15]. Our fitting results show a completely different trend - the Erdogan power model exponent $\alpha$ decreases as the annealing temperature rises. Indeed, this strange behavior has been mentioned when Erdogan proposed the power-law model in the first paper [15], and has been observed in the following studies on fitting thermal decay with the Erdogan power model for both hydrogen-loaded and non hydrogen-loaded fibers, as well as fibers with various dopant compositions $[16,17,32]$. As an example, the result of reference [32] is shown in the inset of Fig 5.7. 
In the above references, the large error was interpreted as arising from the large uncertainty in the measurement of index modulation, which decays to a substantially smaller degree at lower temperatures. However, our experimental datasets, which have significantly improved accuracy due to a much larger thermal decay measurement frequency as shown in Fig 5.6, still leads to the similarly strange behavior of the temperature dependence of exponent $\alpha$. Therefore, the aforementioned explanation is clearly not the real reason for the discrepancy. This weird phenomenon is believed to occur because of the Erdogan power model is not reasonably applicable for describing the thermal decay at higher temperatures $\left(250{ }^{\circ} \mathrm{C}\right.$ and $300{ }^{\circ} \mathrm{C}$, which are the lower temperatures in other published research results) shown in the Fig 5.6. If this model is to be applied, the validity of extrapolating thermal decay of gratings at much lower temperatures (i.e. typical device operating temperature) through the temperature dependence of factors $A$ and $\alpha$ is doubted due to the existence of this error.

As for the other two models, the relationships of the fitting coefficients with the temperature are shown in Fig 5.8. In both cases, it is also difficult to find a rule governing the temperature dependence of the fitting coefficients since they are distributed relatively randomly.

In summary, both the problem of fitting a single isothermal decay curve accurately throughout the entire annealing procedure and that of developing a reasonable dependence of the decay coefficient factors on the annealing temperature lead to a failure of all existing standard decay models to describe the complex thermal decay behavior of fiber Bragg gratings. An advanced method should be developed to analyze the thermal decay characteristics of FBGs, which has to be capable of both fitting the grating decay 
curves at all annealing temperatures (especially at lower temperatures) and forming a reasonable temperature dependence of the fitting coefficients.

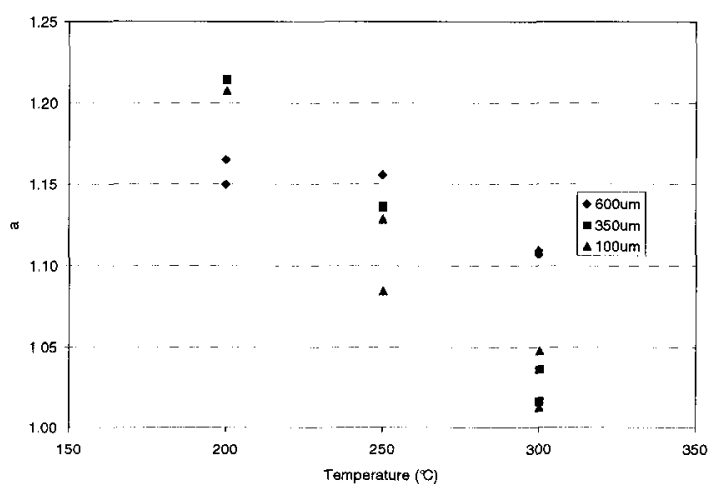

(a)

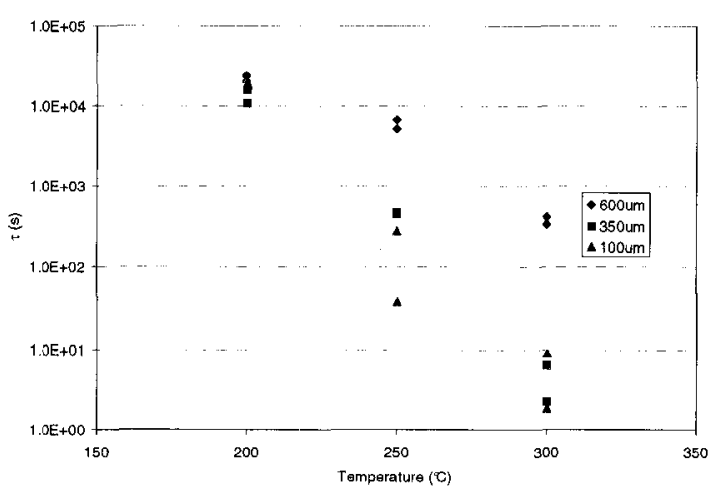

(c)

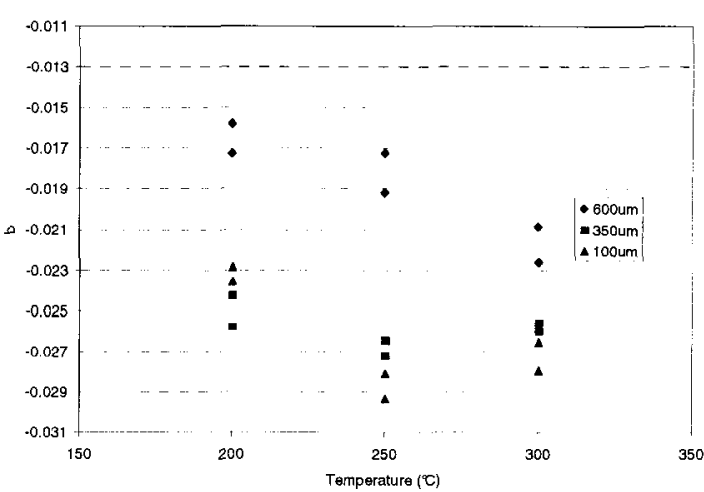

(b)

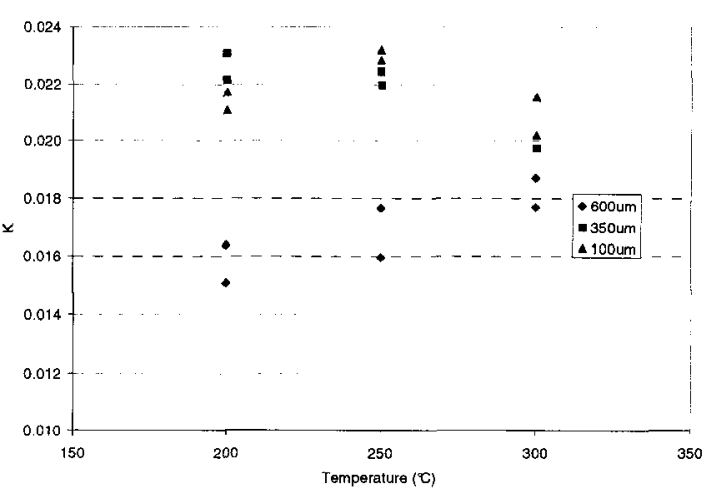

(d)

Fig 5.8 The temperature dependence of the fitting coefficients (a) $a$ and (b) $b$ for simple power model; (c) $\tau$ and (d) $k$ for log time model.

\subsubsection{Validity of the master aging curve model}

As a variation to the Erdogan power law model, the master aging curve model was also proposed by Erdogan to simultaneously analyze all the thermal decay curves at various annealing temperatures, and it was developed as a more general model by Kannan [16]. Assuming that the grating inscription causes a broad distribution of the activation energy of thermodynamically unstable traps, the master aging curve model further designated the 
activation energy rather than the annealing time as the parameter to characterize the advancement of the thermal decay. For a combination of any annealing temperature $T$ and time $t$, the demarcation energy could be expressed as:

$$
E_{d}=K_{B} T \ln (v t)
$$

where $K_{B}$ is the Boltzmann's constant and $v$ the is attempt frequency. By plotting the NICC as a function of $E_{d}$, all the grating erasure data obtained from thermal decay at multiple temperatures will lie on a single master aging curve. The correct attempt frequency $v$ is the optimal value which forces all the experiment datasets to collapse to a single curve. Deduced from the Erdogan power law model, this master aging curve will follow the function:

$$
\eta=\frac{1}{1+\exp \left[\left(E_{d}-E_{0}\right) / \Delta E\right]}
$$

where the center energy $E_{0}$ and the width of defect activation energy distribution $\Delta E$ are the fitting factors obtained by fitting the master curve with the above equation.

Attempts were made to fit the thermal degradation of pre-annealed hydrogen loaded FBGs at temperatures $200{ }^{\circ} \mathrm{C}, 250{ }^{\circ} \mathrm{C}$ and $300{ }^{\circ} \mathrm{C}$ with the master aging curve model. Fig 5.9(a), (b) and (c) show the master aging curves of gratings written at $100 \mu \mathrm{m}, 350 \mu \mathrm{m}$, and $600 \mu \mathrm{m}$ respectively. The values of the attempt frequency $v$ corresponding to the best fit for the grating erasure curves as well as the fitting factors $E_{0}$ and $\Delta E$ are listed in Table 5.1 for the 3 different grating types. 


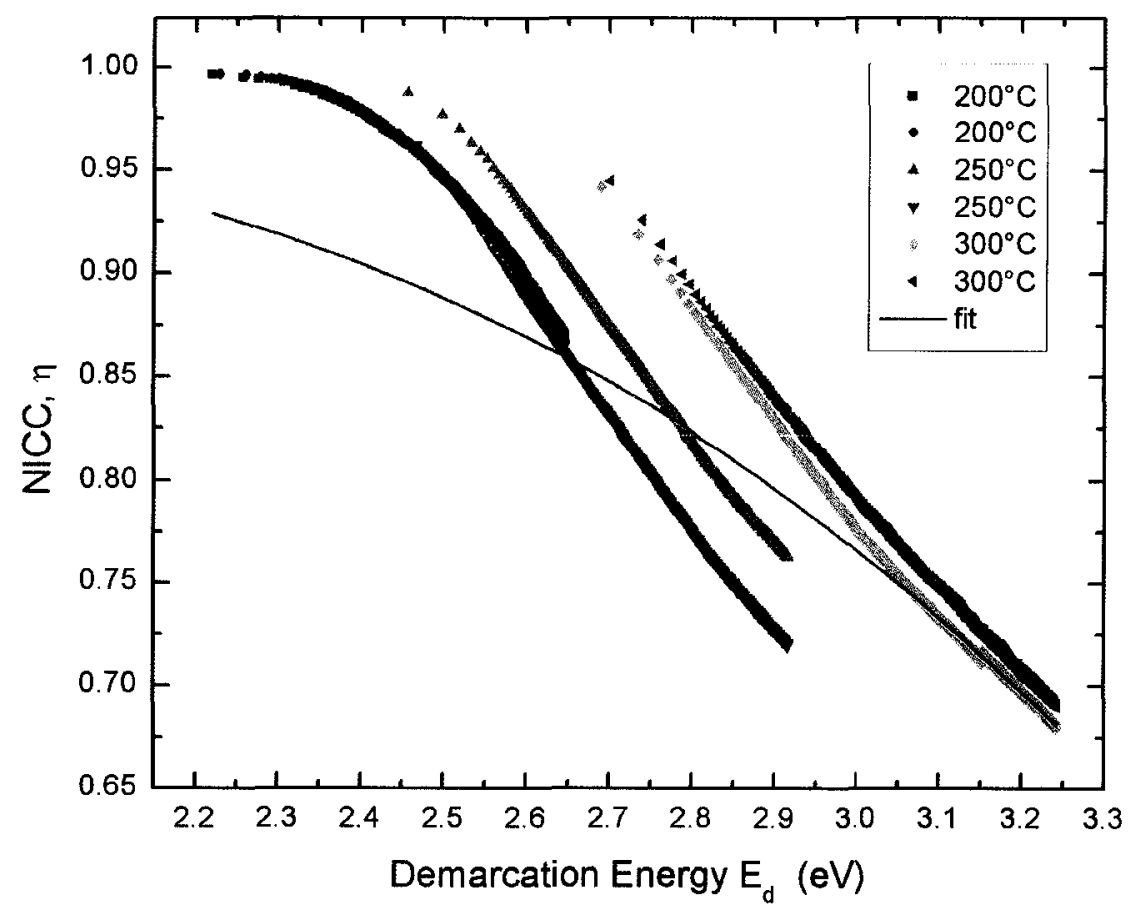

(a)

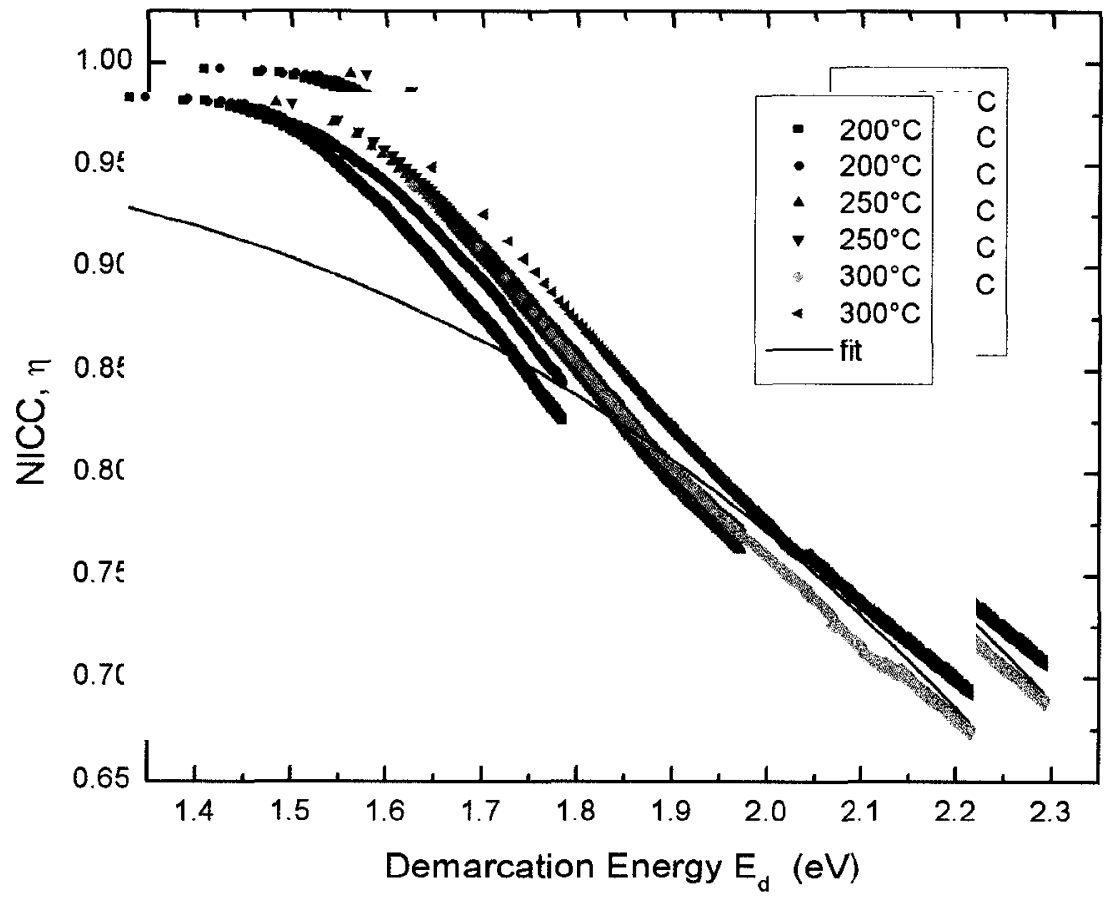

(b)

Fig 5.9 Master aging curves obtained from thermal annealing at $200{ }^{\circ} \mathrm{C}, 250{ }^{\circ} \mathrm{C}$ and 300 ${ }^{\circ} \mathrm{C}$ for pre-annealed gratings written at (a) $100 \mu \mathrm{m}$; (b) $350 \mu \mathrm{m}$. 


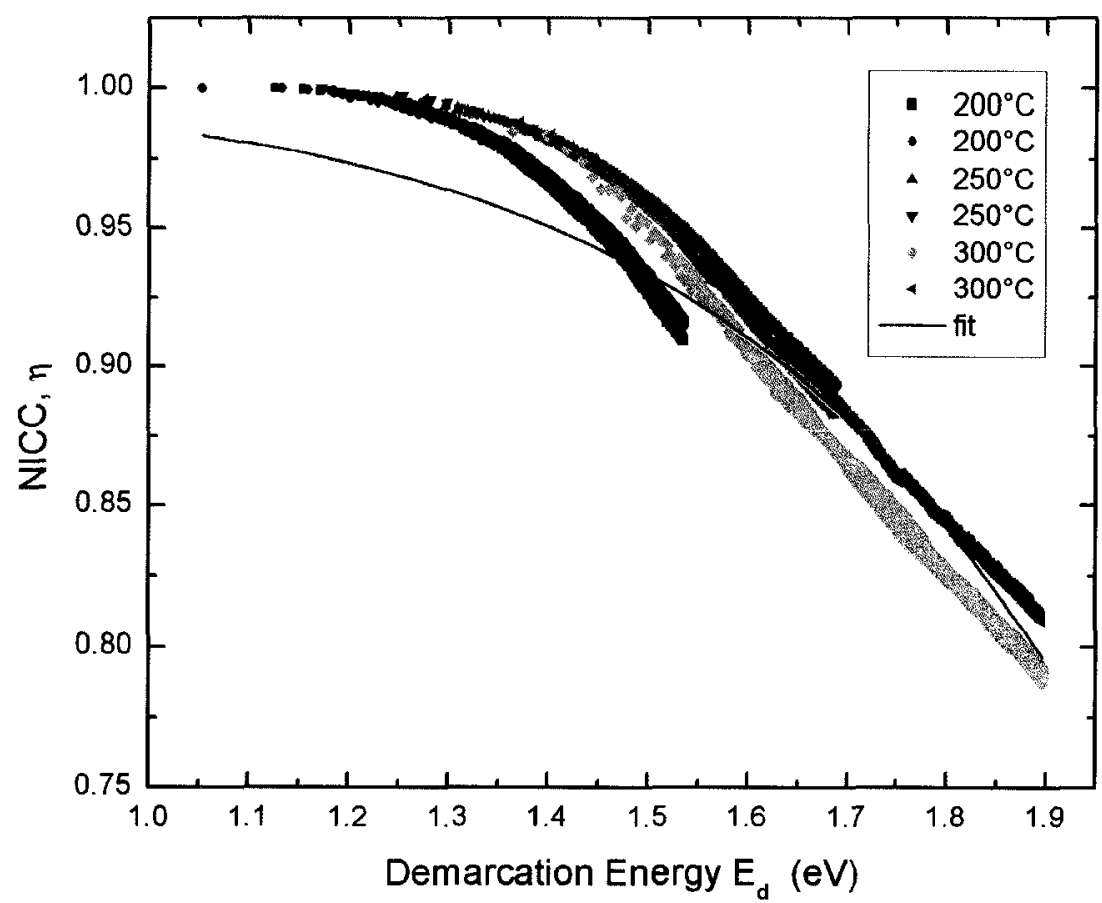

(c)

Fig 5.9 Master aging curves obtained from thermal annealing at $200{ }^{\circ} \mathrm{C}, 250{ }^{\circ} \mathrm{C}$ and 300 ${ }^{\circ} \mathrm{C}$ for pre-annealed gratings written at (c) $600 \mu \mathrm{m}$.

\begin{tabular}{|c|c|c|c|c|}
\hline $\begin{array}{c}\text { Fiber/Mask } \\
\text { Distance }(\mu \mathrm{m})\end{array}$ & $\mathbf{v}(\mathbf{H z})$ & $\mathbf{E}_{\mathbf{0}}(\mathbf{e V})$ & $\Delta \mathbf{E}(\mathbf{e V})$ & $\mathbf{R}^{\wedge} \mathbf{2}$ of Fitting \\
\hline 100 & $1.02 \times 10^{22}$ & 3.6686 & 0.5649 & 0.8933 \\
\hline 350 & $4.57 \times 10^{13}$ & 2.6524 & 0.4434 & 0.9680 \\
\hline 600 & $1.51 \times 10^{10}$ & 2.3199 & 0.3112 & 0.9664 \\
\hline
\end{tabular}

Table 5.1 Fitting results of thermal decay of pre-annealed gratings with master aging curve model.

As shown in the figures, the master aging curve model is not applicable to describe the thermal decay of pre-annealed hydrogen loaded FBGs. For all three types of gratings (different fiber/mask distance), a large deviation between the experimental data and the fitting curve can always be observed in the small demarcation energy range. Indeed, this 
deviation in the small demarcation energy range corresponds to that of Erdogan's power model found in the short term decay according to the mathematical consistence of these two models. Therefore, similar to Erdogan's power model, Equation (5.5) of the master aging curve model is still not an appropriate function for the description of the grating decay.

In addition, the validity of this model is also challenged by the evidence that in certain cases multiple decay data sets at different temperatures can not be collapsed onto a single curve. As shown in the Fig 5.9(a), thermal decay curves of gratings written at $100 \mu \mathrm{m}$ annealed at $300{ }^{\circ} \mathrm{C}$ are separated largely from those decay curves at other annealing temperatures, even though the attempt frequency $v$ has been optimized to reduce the separation of decay curves. This conflicts with the fundamental hypothesis of the master aging curve model that all the decay curves at various temperatures will follow a single curve of NICC against $E_{d}$ as long as the correct attempt frequency $v$ is used. The fact that this hypothesis is not always valid suggests that the decay mechanism of the grating index change is too complex to be explained by the master aging curve model. Either a second parallel reaction process (namely, an additional attempt frequency $v$ ) $[33,45]$ or a different shape of the defect energy distribution [19-20] have been reported to confirm the complex nature of the grating decay.

Another drawback of the master aging curve model is the large-scale variation in the value of the attempt frequency $v$ in different decay experiments. In our experiment, the order of magnitude of $v$ varies from $10^{10}$ to $10^{22}$ for the three groups of gratings with different index contrast, as shown in Table 5.2. Moreover, another strange result encountered during the fitting of the master curve model is that the center activation 
energy of a grating written at $100 \mu \mathrm{m}$ is $3.6686 \mathrm{eV}$, a value that is larger than the center activation energy of a grating written at $600 \mu \mathrm{m}$. This result is not very consistent with the initial idea of this model, which states that gratings written at $600 \mu \mathrm{m}$ should have higher activation energy than those written at $100 \mu \mathrm{m}$ as they show greater thermal stability.

It is common that a comparison of grating activation energy distributions is made between different research results [20]. It is typically concluded that the grating with a higher activation energy distribution will be more thermally stable than others regardless of whether it is obtained by a different analysis. However, it has to be admitted that this comparison is probably meaningless since the important factor of the attempt frequency $v$ is ignored in this simple comparison. Our experimental result is a good example as mentioned above. Indeed, the attempt frequency is always different for different groups of annealing experiment data, even though the gratings are fabricated in the same type of fiber. Therefore, it is believed that master aging curve model is still a mathematical method rather than a physical one depicting the real mechanism behind the thermal decay of fiber Bragg gratings.

\subsection{Comparison with the literature}

Our experimental results have shown that a greater DC refractive index change will improve the thermal stability of grating reflectivity (solely related to the refractive index modulation) to some extent. It has also been shown that the fabrication of FBGs with a lower grating index contrast (AC/DC ratio) by using a large fiber/phase mask distance is an additional way to enhance the thermal stability of a grating. Before our simple 
technology, several groups of researchers have demonstrated that the pre- or postexposure technology with blanket UV beam can efficiently improve the thermal stability for both hydrogenated and nonhydrogenated gratings [21-23]. Here, we will mainly compare our results with reference [22], which provided detailed information of both DC and $\mathrm{AC}$ index change.

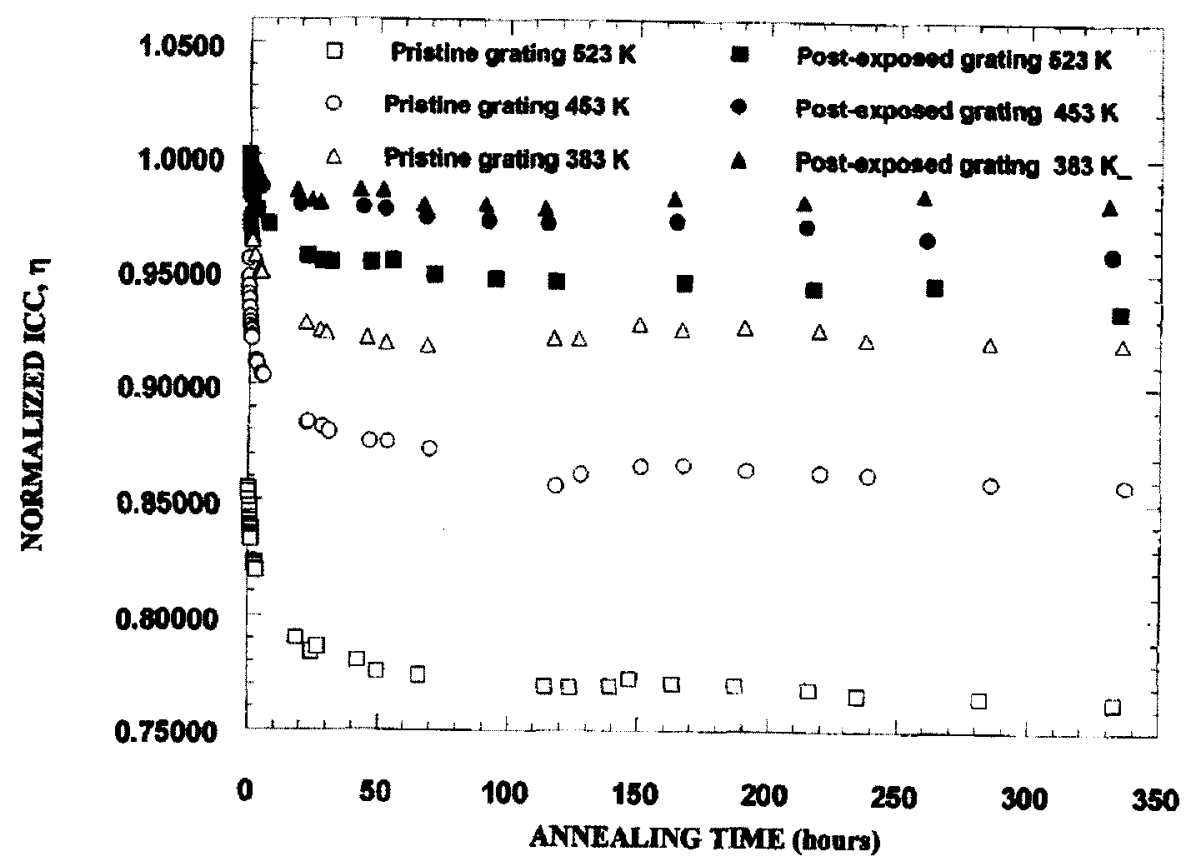

Fig 5.10 Isothermal decay of normalized index modulation as a function of annealing time for both post-exposure gratings and untreated gratings at different temperatures [22].

In that paper, Bragg gratings were written in standard telecom fibers (Corning SMF-28) using light from a pulsed ArF laser at 193nm and employing the phase mask technology. By the end of the illumination process, a refractive index modulation of $\sim 5.3 \times 10^{-5}$ was induced in the gratings. Once these nonhydrogenated gratings were fabricated, some of the gratings were subjected to UV treatment by direct exposure to a uniform UV beam at the same power density as that used for the grating inscription. During this blanket post exposure procedure, the average refractive index change of gratings was monotonically 
increased to $\sim 3.9 \times 10^{-4}$. After that, accelerated aging tests at various temperatures were carried out on both the pristine gratings without any post exposure and the post-exposed gratings.

Fig 5.10 shows the isothermal decay of the normalized refractive index modulation corresponding to both the pristine gratings and the post-exposed gratings annealed at 523 , 453 and $383 \mathrm{~K}$. It is obvious that the post-exposed gratings exhibited significantly increased thermal stability of grating reflectivity compared to the pristine gratings. Similar to our work, this suggests that a largely elevated DC component of the refractive index change in a grating will enhance the thermal stability of grating reflectivity. However, in comparison, the improvement effect in grating stability from increasing the writing distance from $100 \mu \mathrm{m}$ to $600 \mu \mathrm{m}$ is not as pronounced as that obtained with the post-exposure experiments. This discrepancy is actually expected since the grating index contrast employed in the reference is only around 0.136 , which is much lower than that of our gratings fabricated at $600 \mu \mathrm{m}$ (about 0.402 ). If we could inscribe gratings of similar reflectivity as the others used in our comparative test, but at a larger fiber/phase mask distance, thermal stability of grating reflectivity could be further improved to similar levels as the reference. Other factors, such as different fibers (hydrogenated versus nonhydrogenated) and grating index modulations also contribute to the discrepancy in the enhancement of thermal stability.

Another common discovery between our research and the above reference is that the thermal decay of the DC refractive index change has been observed in all gratings. Moreover, the larger the DC component of the grating, the more it decays. Although the evolution of the average index change is difficult to establish during our accelerated 
aging experiments because of the restriction on the accuracy of the grating wavelength measurements, a decrease in the Bragg wavelength is observed for all gratings after thermal annealing at higher temperatures. Table 5.2 lists the Bragg wavelength decrease of all gratings under test compared to the initial wavelength prior to the aging experiment. It can be seen that the annealing-induced Bragg wavelength shift for gratings written at $600 \mu \mathrm{m}$ is larger compared to gratings written at shorter distances when they are subjected to the same annealing process. This is inevitable since that the DC index change formed in the $600 \mu \mathrm{m}$ gratings is substantially larger than their counterparts that were written at shorter distances. This means that the improvement in the thermal stability of the grating reflectivity is made at the expense of the center wavelength.

\begin{tabular}{|c|c|c|c|}
\hline $\begin{array}{c}\text { Fiber/Mask } \\
\text { Distance }(\boldsymbol{\mu m})\end{array}$ & $\begin{array}{c}\Delta \lambda(\mathbf{n m}) \text { after } \\
\text { aging test of } \\
\mathbf{2 0 0} \mathbf{~}^{\circ} \mathbf{C}\end{array}$ & $\begin{array}{c}\Delta \lambda(\mathbf{n m}) \text { after } \\
\text { aging test of } \\
\mathbf{2 5 0}{ }^{\circ} \mathbf{C}\end{array}$ & $\begin{array}{c}\Delta \lambda(\mathbf{n m}) \text { after } \\
\text { aging test of } \\
\mathbf{3 0 0} \mathbf{C}\end{array}$ \\
\hline 600 & 0.13 & 0.16 & 0.29 \\
\hline 600 & 0.14 & 0.19 & 0.3 \\
\hline 350 & 0.13 & 0.16 & 0.23 \\
\hline 350 & 0.09 & 0.11 & 0.2 \\
\hline 100 & 0.07 & 0.09 & 0.16 \\
\hline 100 & 0.08 & 0.12 & 0.15 \\
\hline
\end{tabular}

Table 5.2 The decrease in the Bragg wavelength induced by the accelerated aging experiment on pre-annealed gratings.

As demonstrated in our experiment, gratings written at $600 \mu \mathrm{m}$ with larger mean refractive index change have higher thermal stability than those at $100 \mu \mathrm{m}$ and $350 \mu \mathrm{m}$. The enhancement effect of the DC refractive index change on the thermal stability of the gratings has been explained in a way by considering the difference of the thermal stability 
for index changes occurring in the bright and the dark fringes of the interference pattern [22]. It is possible that the thermal decay of a UV-induced refractive index change at the bright fringe is slower than that at the dark fringe, and thus that the thermal decay characteristics of refractive index changes depend on their strength. The higher the induced refractive index change, the slower the decay rate. Yet, this statement contradicts our observation in Section 5.1, where it is suggested that strong gratings follow nearly same thermal decay pattern as weak gratings. One possible reason for this discrepancy is that the difference in the index modulation in that section is not large enough to alter the thermal decay pattern of the grating. In comparison, the mean refractive index change of gratings written at $600 \mu \mathrm{m}$ is about $1.50 \times 10^{-3}$, which is two and half times larger than its counterpart at $100 \mu \mathrm{m}$. As the refractive index change at the bright fringe of a grating written at $600 \mu \mathrm{m}$ is much larger than that of the $100 \mu \mathrm{m}$ grating, it is possible that the difference in the thermal stability of the two refractive index changes could now be exhibited.

Besides the above explanation, we propose an alternative way to interpret the improvement in the thermal stability of the reflectivity of the grating. It is enlightened by the observation of grating growth behavior when saturated gratings are fabricated in hydrogen loaded fibers. Fig 5.11 shows the grating growth behavior during the formation of saturated hydrogen loaded FBGs, i.e. for gratings where the reflectivity reaches a maximum value and then levels off or decreases. All grating inscription conditions are the same as in Chapter 3. It is particularly interesting to note that in the present case, the total UV-induced index change does not saturate and remains positive on average, and 
the AC component does not decrease to zero: this behavior is different from the commonly observed Type IA and Type IIA gratings [46-47].

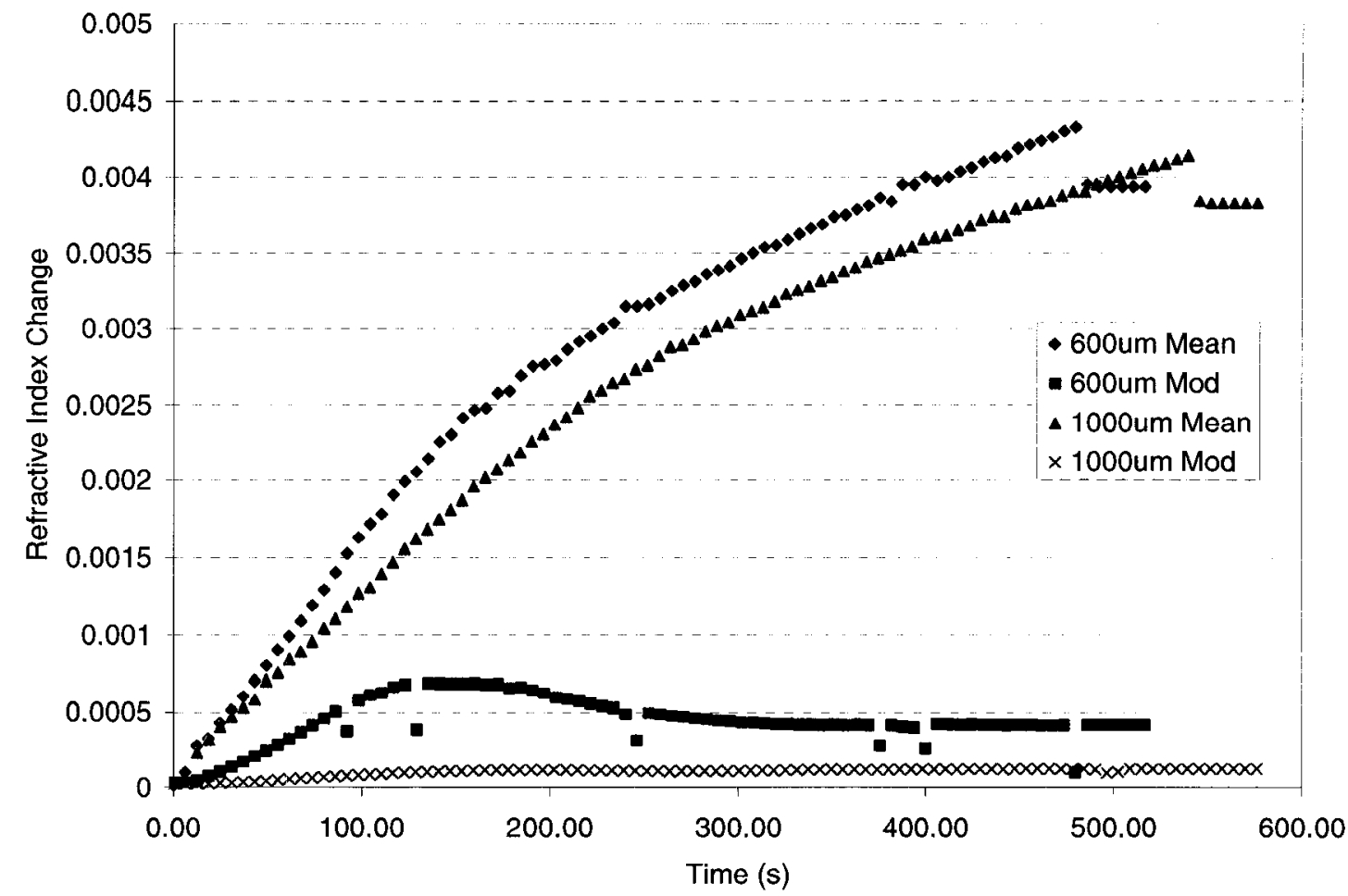

Fig 5.11 The growth of both the mean refractive index change and refractive index modulation during the formation of saturated gratings written at both 600 and $1000 \mu \mathrm{m}$.

In Fig 5.11, a long exposure after the grating reflectivity saturation leads the grating to a new regime, where the average refractive index change keeps increasing linearly but the $\mathrm{AC}$ index change remains approximately constant. This surprising result suggests that the refractive index change induced by UV light on hydrogen-loaded standard telecom fiber involves a second mechanism, which is "non-local" in the sense that it does not allow variations on the scale of the grating period (half a micrometer) but is responsible for a continuing growth of the average refractive index change. Such "local" versus "global" photosensitivity phenomena were observed previously in defect population of gratings

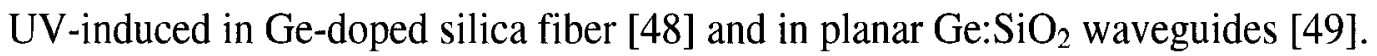


When hydrogen loaded fiber gratings are subjected to the accelerated aging test, the DC component of the grating index change, part of which is formed from the non-local mechanism, decays faster than the AC component. We believe that the thermal decay of the large average index change will eventually prevent the decay of the refractive index modulation. This will occur because a larger portion of the possible reverse pathways of the UV-induced defects responsible for the refractive change are being removed when the DC components of the index change has annealed out. As a result, a larger DC refractive index change formed in a hydrogen loaded fiber will lead to a more thermally stable grating reflectivity (which comes solely from the index modulation).

However, there is only one doubt about the above proposal of a second mechanism. Note that all gratings under accelerated aging tests are unsaturated rather than saturated. The above explanation is based on the assumption that both mechanisms, the global and the local refractive index change, should also occur simultaneously in the linear grating growth region. Furthermore, the portion of contribution to the average UV-induced refractive index change from each mechanism is dependent on the intensity distribution of the interference fringe pattern (and accordingly, the writing distance). A frustrating point about this assumption will be the observation that the grating index contrast is nearly one for gratings written at shorter distances such as $50 \mu \mathrm{m}$ and $100 \mu \mathrm{m}$. This means that the average refractive index change for these gratings is entirely the result of index modulation amplitude (namely, the local refractive index change effect), and no second mechanism is involved during the fabrication. It is not plausible that a global refractive index change could be induced by exposure to a partial contrast interference fringe but not to a total contrast interference fringe. 


\section{Chapter 6}

\section{Conclusions and Recommendations for Future}

\section{Work}

One of the goals of this thesis was to determine the relationship between the thermal stability of a fiber Bragg grating and its AC/DC index contrast by changing the fiber/phase mask distance. In addition, it was also aimed at explaining the erratic thermal stability results of the mass produce FBGs in the industry.

Based on the accelerated aging test results of hydrogen-loaded pre-annealed FBGs, main conclusions from this work are as following:

1. No significant thermal stability variation is observed for weak gratings written at different fiber/mask distances, for which both the index modulation and the average index change are relatively small.

2. For strong gratings, thermal stability of grating reflectivity increases only for large $\mathrm{DC} / \mathrm{AC}$ index ratios (small $\mathrm{AC} / \mathrm{DC}$ ratios) and these only occur for large fiber/phase mask distances.

- Since the thermal stability of gratings written at $100 \mu \mathrm{m}$ and $350 \mu \mathrm{m}$ fiber/mask distance are nearly the same, any small error in fiber/mask distance is not the source of stability variations in mass produced FBGs.

- A two-step process (pre- or post-exposure of the gratings with a uniform UV laser beam) appears to improve the thermal stability of the grating reflectivity more 
than our one-step fabrication process. We believe that fabricating gratings at even larger fiber/mask distances than those used in our experiments (and therefore obtaining similar index contrast ratios to the two-step process) would improve the thermal stability to a similar level as obtained with the two-step process. However, the reproducibility of grating at this fairly large distance may become poor due to the variation in the spatial coherence of the UV laser source.

3. Nominally identical gratings show a relatively large variation in their thermal decay during the same accelerated aging tests, and the thermal decay behavior is different depending on whether the analysis is performed relative to the as-written or to the pre-annealed gratings. As the identical hydrogen-loaded gratings without the preannealing procedure under accelerated aging tests show almost identical decay behavior, the uncontrolled pre-annealing process using the heat gun may be the source of variation.

4. For our gratings, none of the accepted models of thermal decay fit all of the experimental data sets. As a result, the lifetime of the grating at the operational temperature can not be predicted without an appropriate decay model. Only qualitative prediction of thermal stability is possible.

5. We observed anomalous growth patterns of gratings fabricated on hydrogen-loaded standard telecom fibers, which have never been reported before for FBGs: saturation of the index modulation $\Delta n_{\text {mod }}$ (keeping constant after saturation) and continuous growth of the average index change $\Delta n_{\text {mean }}$. This may lead to a new interpretation of the thermal decay involving separate process for $\Delta n_{\text {mod }}$ ("local" change) and $\Delta n_{\text {mean }}$ ("global" change). See details in Chapter 5. 
As for the future work, several possible steps are suggested as follows:

1. Perform additional thermal decay experiments on hydrogen-loaded gratings using a controlled pre-annealing procedure (such as quick annealing in a high-temperature oven) to determine if this is the source of the variations observed.

2. Review all the decay data and attempt to develop a double- or multiple-process decay model characterizing the decay behavior of our gratings.

3. Explain and fully characterize the novel grating growth pattern observed. 


\section{Bibliography}

[1] K. O. Hill, Y. Fujii, D. C. Johnson, and B. S. Kawasaki, "Photosensitivity in optical fiber waveguides: Application to reflection filter fabrication," Appl. Phys. Lett., vol. 32, pp. 647-649,1978

[2] A. Othonos and K. Kalli, Fiber Bragg Gratings: Fundamentals and Applcations in Telecommunications and Sensing, Boston: Artech House, 1999

[3] R. Kashyap, Fiber Bragg Gratings, San Diego: Academic Press, 1999

[4] H.N. Baker, S.R. Baker, V. Baker, R.S. Baulcomb, K.C. Byron, S.J. Clements, T.J. Cullen, S. Dvavis, A. Fielding, and D. Goodchild, "A low loss 4-channel wavelength de-multiplexer based on fiber Bragg gratings”, Proc. ECOC'96., Oslo, Norway, WeD 1.7, pp.151-154, 1996

[5] C.R. Giles, "Lightwave applications of fiber Bragg gratings," J. Lightwave Technol., vol. 15, pp.1391-1404, 1997

[6] F. Bilodeau, D.C. Johnson, S. Theriault, B. Malo, J. Albert, K.O. Hill, and C.R. Giles, “An all-fiber dense wavelength-division multiplexer/demultiplexer using photoimprinted Bragg gratings," IEEE. Photon. Techonl. Lett., vol. 7, pp.388-390, 1995

[7] A. Othonos, X. Lee, and D.P. Tsai, "Spectrally broadband Bragg grating mirror for an erbiumdoped fiber laser," Opt. Eng., vol. 35, pp.1088-1092, 1996

[8] J.L. Zyskind, V. Mizrahi, D.J. DiGiovanni, and J.W. Sulhoff, "Short single frequency erbiumdoped fibre laser," Electron. Lett., vol. 28, pp.1385-1387, 1992

[9] L. Dong, W.H. Loh, J.E. Caplen, J.D. Minelly, K. Hsu, L. Reekie, "Efficient single-frequency fiber lasers with novel photosensitive Er/Yb optical fibers," Opt. Lett., vol. 22, pp.694-696, 1997

[10] J.A.R. Williams, I. Bennion, K. Sugden, and N.J. Doran, "Fiber dispersion compensation using a chirped in fiber Bragg grating," Electron. Lett., vol. 30, pp.985-987, 1994

[11] R. Kashyap, S.V. Chernikov, P.F. Mckee, and J.R. Taylor, "30ps chromatic dispersion compensation of $400 \mathrm{fs}$ pulses at 100Gbits/s in optical fibres using an all fibre photoinduced chirped reflection grating," Electron. Lett., vol. 30, pp.1078-1080, 1994 
[12] A.D. Kersey, M.A. Davis, H.J. Patrick, M. LeBlanc, K.P. Koo, C.G. Askins, M.A. Putnam, and E.J. Friebele, "Fiber grating sensors," J. Lightwave Technol., vol. 15, pp.1442-1463, 1997

[13] K.O. Hill, and G. Meltz, "Fiber Bragg grating technology fundamentals and overview," J. Lightwave Technol., vol. 15, pp.1263-1276, 1997

[14] Y.J Rao, "In-fibre Bragg grating sensors," Meas. Sci. Technol., vol. 8, pp.355-375, 1997

[15] T. Erdogan, V. Mizrahi, P.J. Lemaire, and D. Monroe, "Decay of ultraviolet-induced fiber Bragg gratings", J. Appl. Phys., vol. 76, pp.73-80, 1994

[16] S. Kannan, J. Guo, and P.J. Lemaire, "Thermal stability analysis of UV-induced fiber Bragg gratings", J. Lightwave Technol., vol. 15, pp.1478-1483, 1997

[17] S. Baker, H. Rourke, V. Baker, and D. Goodchild, "Thermal decay of fiber bragg gratings written in boron and germanium codoped silica fiber", J. Lightwave Technol, vol. 15, pp.1470-1477, 1997

[18] S. Ishikawa, A. Inoue, M. Harumoto, T. Enomoto, and H. Kanamori, "Adequate aging condition for fiber Bragg grating based on simple power law model", Proc. OFC'98, pp.183184,1998

[19] J. Rathje, M. Kristensen, J.E. Pederson, "Continuous anneal method for characterzing the thermal stability of ultraviolet Bragg gratings", J. Appl. Phys., vol. 88, pp.1050-1055, 2000

[20] N.K. Viswanathan and D.L. LaBrake, "Accelerated-aging studies of chirped Bragg gratings written in deuterium-loaded germano-silicate fibers", J. Lightwave Technol., vol. 22, pp.19902000,2004

[21] E.Salik, D.S. Starodubov, V. Grubsky, and J. Feinberg, "Thermally stable gratings in optical fibers without temperature annealing," Proc. OFC'99, pp.56-58, 1999

[22] Q. Wang, A. Hidayat, P. Niay, and M. Douay, "Influence of Blanket Postexposure on the Thermal Stability of the Spectral Characteristics of Gratings Written in a Telecommunication Fiber Using Light at 193 nm", J. Lightwave Technol., vol. 18, pp.1078-1083, 2000

[23] M. Aslund, and J. Canning, "Annealing properties of gratings written into UV-presensitized hydrogen-outdiffused optical fiber," Opt. Lett., vol. 25, pp.692-694, 2000 
[24] D. Lam, and B. Garside, "Characterization of single-mode optical fiber filters", Appl. Opt., vol. 20, pp.440-445, 1981

[25] T. Erdogan, "Fiber grating spectra", J. Lightwave Technol., vol. 15, pp.1277-1294, 1997

[26] A.W. Snyder, J.D. Love, Optical Waveguide Theory, New York: Chapman and Hall, 1983

[27] P.E. Dyer, R.J. Farley, and R. Giedl, "Analysis of grating formation with excimer laser irradiated phase masks", Opt. Comm., vol. 115, pp.327-334, 1995

[28] I. Riant, S. Borne, and P. Sansonetti, "Dependence of fiber Bragg grating thermal stability on grating fabrication process", Proc. OFC'96, paper Tu05, pp.86-87, 1996

[29] L. Dong, and W.F. Liu, "Thermal decay of fiber Bragg gratings of positive and negative index changes formed at $193 \mathrm{~nm}$ in a boron-codoped germanosilicate fiber", Appl. Opt., vol. 36, pp.8222-8226, 1997

[30] K.E. Chisholm, K. Sugden, and I. Bennion, "Effects of thermal annealing on Bragg fibre gratings in boron/germania co-doped fibre", J. Phys. D: Appl. Phys., vol. 31, pp.61-64, 1998

[31] G. Brambilla, and H. Rutt, "Fiber Bragg gratings with enhanced thermal stability", Appl. Phys. Lett., vol. 80, pp.3259-3261, 2002

[32] S. Pal, J. Mandal, T. Sun, and K.T.V Grattan, "Analysis of thermal decay and prediction of operational lifetime for a type I boron-germanium codoped fiber Bragg grating", Appl. Opt., vol. 42, pp.2188-2196, 2003

[33] S. Pal, T. Sun, K. Grattan, S. Wade, S. Collins, G. Baxter, B. Dussardier, and G. Monnom, "Bragg gratings written in Sn-Er-Ge-codoped silica fiber: investigation of photosensitivity, thermal stability, and sensing potential", J. Opt. Soc. Am. A, vol. 21, pp.1503-1511, 2004

[34] S. Pal, "Characterization of thermal (in)stability and temperature-dependence of type-I and type-IIA Bragg gratings written in B-Ge codoped fiber", Opt. Comm., vol. 262, pp.68-76, 2006

[35] D. Razafimahatratra, P. Niay, M. Douay, B. Poumellec, and I. Riant, "Comparison of isochronal and isothermal decays of Bragg gratings written through continuous-wave exposure of an unloaded germanosilicate fiber", Appl. Opt., vol. 39, pp.1924-1932, 2000 
[36] P.Lemaire, R. Atkins, V. Mizrahi, and W.A. Reed, "High pressure $\mathrm{H}_{2}$ loading as a technique for achieving ultrahigh photosensitivity and thermal sensitivity in $\mathrm{GeO}_{2}$ doped optical fibers", Electron. Lett., vol. 29, pp.1191-1193, 1993

[37] B. Poumellec, "Links between writing and erasure (or stability) of Bragg gratings in disordered media", J. Non-Cryst. Solids, vol. 239, pp.108-115, 1998

[38] P. Lemaire, "Reliability of optical fibers exposed to hydrogen: prediction of long-term loss increases", Opt. Eng., vol. 30, pp.780-781, 1991

[39] B. Leconte, W. Xie, M. Douay, P. Bernage, P. Niay, J.F. Bayon, E. Delevaque, and H. Poignant, "Analysis of color-center-related contribution to Bragg grating formation in $\mathrm{Ge}: \mathrm{SiO}_{2}$ fiber based on a local Kramers-Kronig transformation of excess loss spectra", Appl. Opt., vol. 36, pp.5923-5930, 1997

[40] B. Malo, J. Albert, K. O. Hill, F. Bilodeau, and D. C. Johnson, "Effective index drift from molecular hydrogen diffusion in hydrogen-loaded optical fibers and its effect on Bragg grating fabrication", Electron. Lett., vol. 30, pp.442-444, 1994

[41] Yuji Masuda, Mitsuo Nakamura, Chisa Komatsu, Keio Fujita, Makoto Yamauchi, Masanori Kimura, Yasuo Mizutani, Susumu Kimura, Yoshifumi Suzaki, Takashi Yokouchi, Kiyoshi Nakagawa, and Seiki Ejima, "Wavelength Evolution of Fiber Bragg Gratings Fabricated From Hydrogen-Loaded Optical Fiber During Annealing", J. Lightwave Technol., vol. 22, pp.934941,2004

[42] A. Hidayat, Q. Wang, P. Niay, M. Douay, B. Poumellec, F. Kherbouche, and I. Riant, "Temperature-induced reversible changes in the spectral characteristics of fiber Bragg gratings", Appl. Opt., vol. 40, pp.2632-2642, 2001

[43] R.J. Egan, H.G. Inglis, P. Hill, A. Krug, and F. Ouellette, "Effects of hydrogen loading and grating strength on the thermal stability of fiber Bragg gratings", Proc. OFC'96, paper Tu03, pp.83-84, 1996

[44] H. Patrick, S.L. Gilbert, and A. Lidgard, "Decay of Bragg gratings in hydrogen-loaded optical fibers", Proc. OFC'95, paper WP10, pp.179-180, 1995 
[45] Y. Shen, J. He, Y. Qiu, W. Zhao, S. Chen, T. Sun, and K.T.V. Grattan, "Thermal decay characteristics of strong fiber high-temperature sustainability", J. Opt. Soc. Am. B, vol. 24, pp.430-438, 2007

[46] Y. Liu, J. A. R. Williams, L. Zhang, and I. Bennion, "Abnormal spectral evolution of fiber Bragg gratings in hydrogenated fibers", Opt. Lett., vol. 27, pp.586-588, 2002

[47] W. X. Xie, P. Niay, P. Bernage, M. Douay, J. F. Bayon, T.Georges, M. Monerie, and B. Poumellec, "Experimental evidence of two types of photorefractive effects occurring during photoinscriptions of Bragg gratings written within germanosilicate fibres", Opt. Commun., vol. 104 , pp.185-195, 1993

[48] T.E. Tsai, G. Williams, and E.J. Friebele, "Uniform component of index structure induced in Ge-SiO $\mathrm{S}_{2}$ fibers by spatially modulated ultraviolet light”, Appl. Phys. Lett., vol. 72, pp. 32423245,1998

[49] J.Canning, and M. Aslund, "Correlation of ultraviolet-induced stress changes and negative index growth in type IIa germanosilicate waveguide gratings", Opt. Lett., vol. 24, pp.463-465, 1999 